Portland State University

PDXScholar

6-4-1997

\title{
ESL CD-ROM Principles and their Application: A Software Evaluation
}

Stephanie Burgi LaMonica

Portland State University

Follow this and additional works at: https://pdxscholar.library.pdx.edu/open_access_etds

Part of the Bilingual, Multilingual, and Multicultural Education Commons Let us know how access to this document benefits you.

\section{Recommended Citation}

LaMonica, Stephanie Burgi, "ESL CD-ROM Principles and their Application: A Software Evaluation" (1997). Dissertations and Theses. Paper 5316.

https://doi.org/10.15760/etd.7189

This Thesis is brought to you for free and open access. It has been accepted for inclusion in Dissertations and Theses by an authorized administrator of PDXScholar. Please contact us if we can make this document more accessible: pdxscholar@pdx.edu. 


\section{THESIS APPROVAL}

The abstract and thesis of Stephanie Bürgi LaMonica for the Master of Arts in Teaching English to Speakers of Other Languages were presented June 4, 1997, and accepted by the thesis committee and the department.

COMMITTEE APPROVALS:
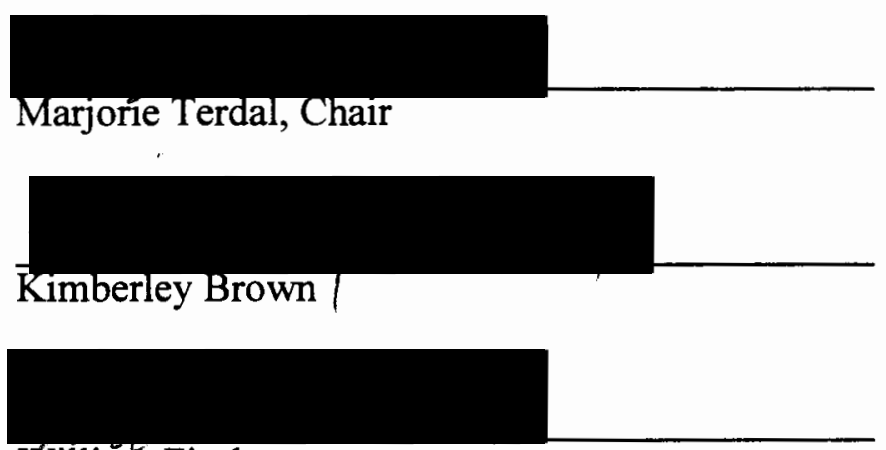

William Fischer

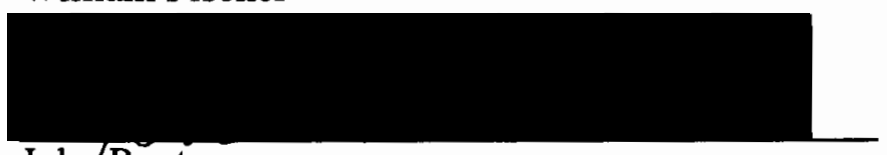

John Rueter

Representative of the Office of Graduate Studies

DEPARTMENT APPROVAL:

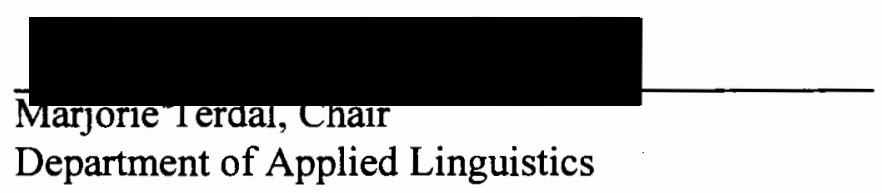

ACCEPTED FOR PORTLAND STATE UNIVERSITY BY THE LIBRARY

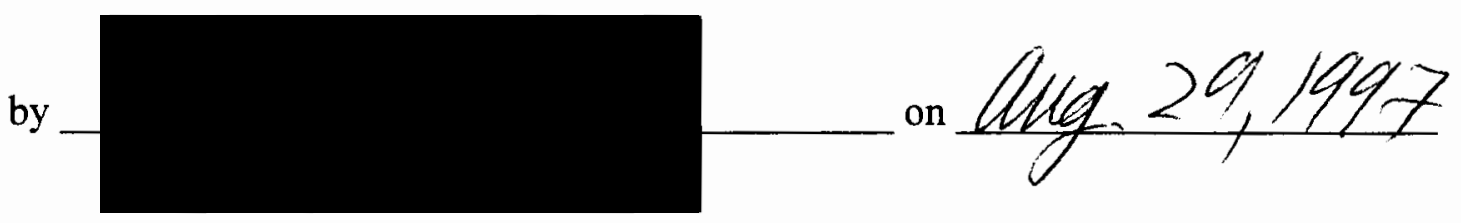




\begin{abstract}
An abstract of the thesis of Stephanie Bürgi LaMonica for the Master of Arts in Teaching English to Speakers of Other Languages presented June 4, 1997.
\end{abstract}

Title: ESL CD-ROM Principles and their Application: A Software Evaluation

CD-ROM applications within the past 5 years have aided the availability of Computer Aided Language Learning (CALL) on a mass consumer level. This study assesses how the instructional design of commercial CALL English as a Second Language (ESL) CD-ROM materials make up for the lack of a human instructional component. The framework within which the evaluation takes place is communicatively based. Using a tool developed specifically for the study, 8 software from 6 companies were systematically evaluated. The 3 -step process followed in the evaluation makes concessions for student, instructional designer, and instructor needs. Research questions address how the areas of user-friendliness, feedback and error treatment, media relevancy, quality of interaction, record keeping, communicative language input and practice, and culture learning are being accounted for in today's CALL materials. Results show today's software has departed little from past behaviorist products. The findings suggest commercial developers are not fully addressing today's communicative teaching approach, that developers might be using other teaching paradigms for the creation of the CALL materials, and that consumers may not be informed enough to demand a more communicative-based computer product. 
ESL CD-ROM PRINCIPLES AND THEIR APPLICATION:

A SOFTWARE EVALUATION

by

STEPHANIE BÜRGI LaMONICA

A thesis submitted in partial fulfillment of the requirements for the degree of

MASTER OF ARTS

in

TEACHING ENGLISH TO SPEAKERS OF OTHER LANGUAGES

Portland State University

1997 


\section{ACKNOWLEDGEMENTS}

Thanks to all the software companies that contributed their products for this study.

Many thanks to Marge Terdal for your support and helping me find my way.

and thank you, bruno 


\section{TABLE OF CONTENTS}

CHAPTER

PAGE

\section{ACKNOWLEDGEMENTS}

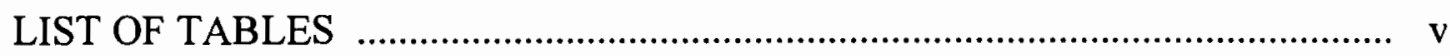

I INTRODUCTION ......................................................................

Background ………........................................................................ 2

Research Questions ................................................................... 13

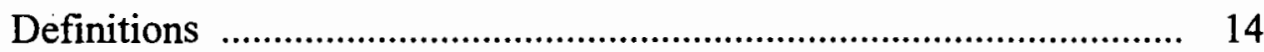

II REVIEW OF THE LITERATURE ………........................................... 17

Today's ESL Classrooms ................................................................. 17

Technology as a Medium of Instruction .............................................. 31

Computers in the Communicative Classroom …….............................. 38

Communicative CALL Material _....................................................... 46

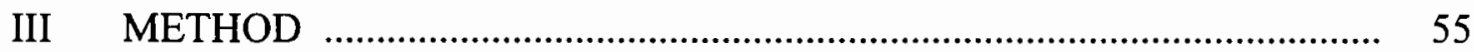

General Design of the Study ............................................................. 56

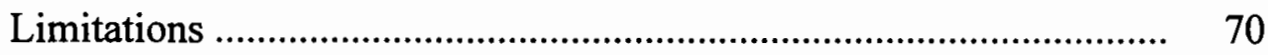

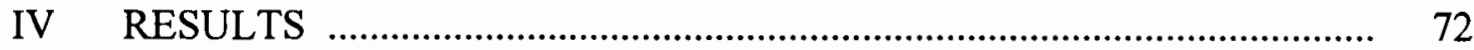

The Evaluations …....................................................................... 75

Interactive Business English .................................................. 75

Dynamic English ................................................................ 81

Let's Go ..................................................................... 87

Learn to Speak English ....................................................... 91

The Rosetta Stone ………………….................................... 98

TriplePlayPlus! ................................................................... 103

ELLIS Senior Mastery ............................................................ 109

Focus on Grammar ........................................................... 115 
V DISCUSSION OF THE RESULTS

Study Overview ..................................................................... 119

Summary of Results .............................................................. 120

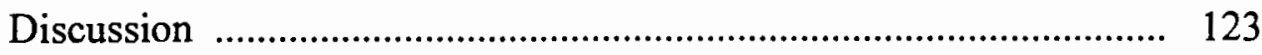

Conclusions and Implications …................................................. 133

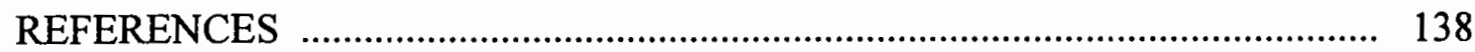

APPENDICES

A SOFTWARE COMPANIES REPRESENTED …............................. 151

B EVALUATION TOOL AND SOURCES ….................................... 152

C SAMPLE NAIVE USER LOG .................................................... 161 


\section{LIST OF TABLES}

TABLE

PAGE

I Software Evaluated in the Study ...................................................... 60

II Matrix of the Findings …................................................................. 73

III Evaluation Components with Good - Excellent Ratings ..................... 120

IV Evaluation Components with Adequate - Weak Ratings ................... 121

V Evaluation Components Totally Lacking …..................................... 122

VI Media Ratings …......................................................................... 123 


\section{CHAPTER I}

\section{INTRODUCTION}

This study is a materials evaluation of Computer Aided Language Learning (CALL) English as a Second Language (ESL) CD-ROM programs available commercially. As the current language teaching approach is communicatively based, the theory behind the approach should guide the creation of materials for the ESL classroom. The instructional design features and content of current CALL materials need to adhere to basic theoretical principles used in today's classrooms if CALL is ever to be disassociated with the earlier CALL programs that are behavioristicallybased. The study mainly assesses the inclusion or lack of instructional design features in the CALL ESL materials that support an environment where a human instructional component is lacking. Content is examined only to discern a general representation of culture and intercultural communication skills for use outside of the classroom.

Eight ESL CD-ROM programs from six companies are represented in the study. A tool developed specifically for the study was administered in a three-step process to each of the software. The evaluation makes concessions for student opinions by looking at the program's user friendliness; instructional designer opinions when looking at the areas of feedback and error treatment, relevance of media, interaction, and record keeping; instructor opinions to discern the representation of culture, culture learning, and communicative language input and use. The findings are in a matrix that provides an overview of the results, and individual software reviews 
describe each program and then report on the findings in relation to the research questions.

The choice of CD's for the evaluation stems from their storage capacities; materials in CD-ROM format now have the potential for a rich, contextual environment for culture learning lacking in earlier products. The use of multimedia allows both a verbal and non-verbal representation of language; this non-verbal representation manifests itself visually through the high quality photos and video lacking in earlier CALL programs. More and more as students in communicative classrooms find themselves one-on-one with a computer that acts not only as the deliverer of information but also the assessor of language use, CALL materials need to promote current communicative theory for the learning of language that can be used in communicative situations outside of the classroom. The instructional design of products needs to break away from the earlier drill-and-practice routines associated with earlier CALL and deliver a product that makes sense for classrooms.

\section{Background}

The advancing technological age we find ourselves in has offered a new medium of instruction to the ESL teacher: the computer. Its arrival onto the language learning scene came at a time when a paradigm shift in ESL was taking place; a move from behaviorist methodology to a communicative methodology was evolving, based on the more current views of language and language learning. Language teaching was moving beyond the mechanical drill and practice routines associated with earlier behaviorist models; instead, the focus in language instruction turned toward the actual use of language in realistic, meaningful, and contextual situations. 
The growth of CALL in some twenty years' time has resulted in large amounts of software, most of it based on the behaviorist paradigm characterized by drill and practice exercises (Schreck \& Schreck, 1991). These exercises required little computer memory, were low in production cost, and could be programmed with the most basic hardware. CALL materials soon became the dumping ground of many "wrong-try-again" computer activities (Mohan, 1992). With the communicative approach guiding state of the art TESOL (see Brown, H.D., 1993; Nunan, 1993; Savignon, 1993), many of the initial programs and behaviorist exercises of CALL no longer support the theoretical grounding of the current teaching approach. In fact, much of today's CALL material is seen as lacking current pedagogical integrity (Liou, 1994; Mohan, 1992).

\section{Current Pedagogy}

To reach pedagogical integrity, CALL programs must reflect the practices and meet the needs of today's ESL classrooms. The Communicative approach to teaching language characterizes current ESL instruction. This approach regards the learning and use of language as strictly for communicative purposes (Celce-Murcia, 1991). Minimally speaking, materials and activities must then provide for meaningful and relevant exchanges of information that the student can use inside as well as outside the classroom. Activities tend toward high levels of interactivity among students, who usually work in groups or pairs. No longer centered around the teacher standing in front of the class, the focus is now on the learners who assume control of their own learning.

A learner-centered classroom is meant to provide a more individualized atmosphere where learning can be tailored to the needs of the student. Realizing the 
various learning styles students bring into the ESL classroom are often ignored under more global class instruction techniques, instructors now strive to support the students by the provision of various modes of input and activities. In a communicative learnercentered environment, learners must be also be allowed the confidence, motivation, and control to succeed. As the goal of today's ESL class is successful self-expression and meaningful negotiation outside of the classroom, students must not feel intimidated to experiment with language. Affective factors such as confidence, motivation and attitude will have an effect on the learner's success rate (LarsenFreeman, 1991). For example, as small group collaborations replace class-front performances, the student can feel less stress during language production. The student's affective filter (Krashen \& Terrell, 1983) is thus thought to be lowered, resulting in a more relaxed state for the student.

Culturally, today's ESL classrooms are environments prone to a mingling of various backgrounds. By its very nature, the ESL class is embarking on an intercultural experience. As students learn linguistic formulas with which to communicate, they also learn the associated culture underlying the linguistic use. Teaching students how to become culturally aware of themselves and others as bearers of culture is increasingly seen as a necessary component. Incorporating more of a theory base that treats culture as something other than "food, flags, or festivals" (Brown, K., 1995), many of today's classrooms are also beginning to treat culture as a more integrated component.

When comparing the needs of a communicative classroom to what current computer aided instruction can offer, there exists an excellent match in many areas: individualized instruction, learner control, the potential motivation of working and learning with current technology, and contextualized language use. These factors can contribute to a positive learning environment. To meet the needs of today's students 
and classrooms, it is up to instructional designers and producers of CALL materials to endow the software with a pedagogical validity previously lacking.

The shortcomings of CALL materials can rightfully be attributed to their limited pedagogical worth. After all, the choice of materials is based on underlying theories of language and language learning, and on the needs of both students and teachers (Brown, J. D., 1995). As mentioned earlier, the drill-and-practice exercises of many CALL programs no longer fit today's theory of language and language learning, nor do they fit the needs of students and teachers. Drill and practice programs decontextualize language learning by focusing more on linguistic form instead of communicative function. A richer, more contextualized environment similar to that found in first language acquisition is desired as today's students and instructors aim their goals toward language use and communication. Not only must the linguistic and sociolinguistic environment be supported in CALL materials, but the material needs to provide an environment that allows students to learn and not feel confused or lost in the face of technology. Since the computer must provide for a non-human instructional component, issues like program user-friendliness, material relevance, types of feedback, and quality of interaction begin to play larger roles in CALL material than ever before.

\section{Current Technology}

As theoretical instructional changes characterize the ESL classroom, technological changes are offering alternatives that also can shape the current face of CALL. The computer technology of the 1990's offers an alternative to the drill and practice environments associated with earlier CALL programs. One of the largest breakthroughs is based on storage. The increase in software storage permits the inclusion of rich visuals; quality photo images can now be incorporated into software 
programs to offer an immediate source of visual input. More recently, video has been converted to digital data and thus made ready for computer applications. Carefully chosen authentic video by itself can supply the viewer with detailed sociocultural and sociolinguistic contexts of language use. Seeing language use in real-world situations provides a source of cultural material for today's communicative learning environment (Olshtain \& Cohen, 1991).

Beyond video, the combination of photos, sound, graphics, animation, and text makes possible a linguistically, culturally, and contextually rich learning environment lacking in earlier CALL products. The older decontextualized CALL activities can now be placed within a culturally meaningful context. In 1990 Kenning and Kenning asserted that the computer was a "drawback" for language teaching; at that time CALL materials were unable to "provide the kind of rich, immediate contextualization offered, for instance, by video, where the ability to both hear and see instances of authentic language use gives access to important and useful information" (1990, p. 40). In 1997 the "useful information" referred to by Kenning and Kenning can now be incorporated into CALL material. Students can now have a closer look at how language is used outside of the classroom through the media of photographs or video.

The storage capacity of CD-ROMs facilitates the incorporation of text, graphics, animation, photos, and video in multimedia materials. Overall the disks can hold the equivalent of 250,000 pages of text, which is the equivalent of data stored on 550 high-density floppy disks (Brock, 1994, p. 43). As photos, video, and animation can quickly create large amounts of data to be stored, a data compression process reduces their storage space considerably; $\mathrm{CD}$ storage capacity makes the $\mathrm{CD}$ a perfect container for multimedia products and applications. Brock (1994) states that "because of the benefits of digitization, product variety, and storage capacity of $\mathrm{CD}$ technologies...by the year 2000 probably $90 \%$ of software purchased for schools will 
be presented on CD technology media rather than computer diskettes" (p. 148). Recent improvements in storage have resulted in the DVD-ROM, which has a 4.7 gigabyte (GB) storage capacity. This amount of storage is more than seven times the amount of current CDs (Brown, E., 1997). $17 \mathrm{~GB}$ versions are in the making.

\section{Current Materials}

While current technology offers the potential for better contextualized language presentation and practice, it is important to realize that "in the end, it's not the technology itself that counts -- it is what you put into it, the content" (Harland, 1991, p. 159). With the communicative approach guiding ESL material design for the past twenty years, it is likely that the organization of material content (i.e. the scope and sequence) is better understood than the delivery of content through the computer which produces the new need of components making up for the lack of a visible human instructor. Schreck and Schreck (1991) advise such a focus on the delivery of content as well as on the learning environment of the program. They say that it is largely these two features, the delivery of content and the learning environment, that will separate a quality program from being just a workbook in digitized form.

The instructional designers of today's CALL CD-ROM tutorial materials must consider the interaction provided and learning environment created by their program. The multimedia and the programming of instructional design features must be relevant and useful. Unfortunately, this is not always the case. There is an assumption, especially among first time CALL users and evaluators, that all media elements must be present, and that all these elements together constitute a quality state of the art CALL program (Schreck \& Schreck, 1991). Yet the use of combined media in multimedia applications does not automatically create an improved CALL product. 
As a result, the media elements have the potential of being misused, over-used, and possibly detracting from the learning objectives of the program.

If less knowledgeable CALL users expect a general inclusion of all the multimedia components, developers may get the message that consumers of their products look more for the antics of the software than the instructional integrity. Brinton (1991) advises that all media material needs to have a relevance and importance; media use must be carefully planned and interwoven with the other lesson components. The role each medium plays must be thoughtfully considered so that the media can effectively support learning objectives. If used properly, today's multimedia CALL materials can provide multiple channels through which learning can occur (a reference to the learning needs of students in today's communicative classroom that will be explained in chapter II). Ineffective use of media and poor instructional design in CALL will only perpetuate the lack of pedagogical value of materials in the future.

Current marketing of materials also can leave some doubt about the materials' pedagogical integrity (i.e. the education level of the product compared to the entertainment level). Product literature found on the Internet through a language software site provides an insight into how marketers believe they will attract buyers. Software features like "amusing photographs," "natural sounding audio," and "interactive exercises" dot the existing marketing material. Some companies attempt to entice the reader with statements like "education and entertainment are not the same." This same company, itself having numerous ESL titles available, goes on to say that their "goal is to motivate and engage students." Whether or not the product is motivating and engaging, how motivation and engagement are achieved, and how the product offers educational value are left to the interpretation of the reader. In short, the marketing literature appears to offer reassurance of a good product through the use 
of "buzzwords" like "interactive," "motivation," "effective," "proficiency oriented." At a glance these words look substantial and relevant to today's ESL needs. But product literature leaves many questions unanswered in regard to today's classroom and the instructional design issues making up the non-human instructional component.

The creation of software is an expensive investment of time and money; programmers joining the multimedia movement and perhaps not fully familiar with ESL pedagogy have littered the ESL markets with too many software packages having too little instructional value for today's ESL environments (Liou, 1994; Mohan, 1992). This is a huge problem in itself, for not only do most of the programs not follow sound pedagogical guidelines, the material, "if it's over promoted, will rapidly bring the whole CALL enterprise into disrepute" (Thomas, 1986, p. 119). The negative advertising from the unsound products due to untrained ESL programmers may provide fuel for educators and administrators unwilling to take an honest look at CALL in ESL instruction.

As the education market is not financially available to attract programmers needed for the courseware (Conrad, 1996), some action needs to be quickly taken to raise an awareness of current pedagogical issues in software. In turn, a better product can be demanded. Experienced ESL instructors can, of course, provide the needed underlying theoretical knowledge for the programs. Yet many educators may not have the time to commit to software creation; some are even unwilling to help at all, citing lack of the required technical skills as a reason (Ganszauge, Hult, Sajavaara, \& Kottinen, 1994). It would be a great misfortune to relegate the use of computers in the ESL classroom to the status of another technologically-based medium of instruction found in the Audio Lingual Method (ALM). Yet if no worth past what teachers can already provide can be discerned from CALL, and if the materials are continually 
categorized as having little or no pedagogical value, CALL could go by the wayside like the ALM.

\section{Current Attitudes}

Because of the changing views of language learning and teaching, the behaviorist-based Audio Lingual Method has left a disdain for technology that is still found over thirty-years later in the 1990's. Bedford (1991) suggests that CALL materials and their associated methodology are being compared to the ALM's drill and practice methodology, which required little teacher involvement. She states that "some teachers are worried that the computer is a new incarnation of the teaching machine" (pp. 170 - 171). Bedford is referring to the use of a tape-recorder as the deliverer of content. The teacher's role in an ALM class was minimal; the materials and activities were predetermined, thus requiring little teacher involvement or control.

Bedford gives no empirical evidence to support her statement, but she is not the only one who feels that the failure of the audiolingual method may affect the technological orientation of ESL instructors. Al-Arishi (1994) agrees: "Perhaps the once-burned, twice-shy mentality [from ALM] has conditioned the slow, often adverse, response...to the newer technology centered around the computer" (p. 177). Negative attitudes toward technology and CALL, no matter how previously formed, do not promote the positive teacher support needed for the creation of sound CALL materials and for the demand of sound materials from publishers.

While both Bedford and Al-Arishi portray educators as hesitant about today's technology, there may be some truth to Bedford's and Al-Arishi's assertions. This study does not focus on instructors' attitudes. It does, however, focus on the need for CALL materials to be designed in a way that alleviates instructors' fears about the quality of instruction taking place when the computer is in control of content and its 
delivery. Beyond the hesitancy alluded to by Bedford and Al-Arishi, there is a more justifiable and realistic attitude toward CALL materials: "Teachers justifiably wonder how computers can fit into the kind of teaching prevalent in today's classroom, both at a practical and theoretical level" (Kenning \& Kenning, 1990, p. 12).

From a CALL perspective Hubbard (1992) reinforces Larsen-Freeman and Long's assertion (1991) that theory is necessary in order to provide for a sound pedagogy. In short, there must be cohesion in theory within and throughout the software. A communicative classroom considers the communicative use of language; the associated methodology of a communicative classroom must then be present in the courseware, modified to technology's capabilities. Beyond the methodology there must be an assurance of quality instruction and built in instructional features that support the learning environment without a visible human instructor present. Having a consistent theory base will then provide criteria with which to evaluate the software. Layne and Lepeintre (1996) thus encourage programmers to keep up with current pedagogical practices.

\section{Conclusion}

This study is based on an evaluation of CALL CD-ROM multimedia materials for use in the communicative classroom. The evaluation will examine the software's pedagogical worth, or lack of it, through the presentation and design of the materials' content when delivered by a non-human instructor. The evaluation will consider current theories of language learning and whether those theories are applied in the CALL material. Computers and software should never be thought of as replacements for the language instructor. Yet the technology must, as a medium of instruction, account for a number of components that are provided by today's instructors, non- 
CALL materials, and classroom learning environments. These components are: input that is meaningful, comprehensible and motivational; error recognition and analysis; provision of feedback; communicative language use and practice; student interaction with the material; culture learning; environment as conducive to learning; monitoring of student progress. Without attention to these components as well as the inclusion of culture learning, communicative CALL may never achieve a meaningful place in existing methodology. Besides providing an overview of today's materials, this study will illuminate the potential of today's technology and whether it is being used to its fullest to meet current ESL needs.

The timeliness of this study cannot be overlooked. The medium of computers has the potential of providing a linguistically and sociolinguistically rich environment where learning can take place. Through the development of the storage capacities of CD-ROMs, the learning environment has the capability of being even more robust. Unfortunately much of today's software may still not meet the pedagogical requirements of the communicative classroom. An evaluative survey of available CDROM ESL software will shed light on current pedagogical value and worth of the instructional design, and how the CALL material makes up for the lack of a humancomponent in its presentation. With the technology available today, there is no excuse for the decontextualized "drill and practice" material found in early CALL programs. Unless instructional design begins to get more involved with technical potentials and the current needs of the communicative classroom, CALL materials, like most computer software, will have a very short shelf life. 
Research Questions

1. How do today's commercial CD-ROM ESL materials account for the non-human instructional component? The components to be considered are (in no specific order): provision of feedback and error treatment; relevance of media as input; surface feature relevancy (i.e. borders, highlighting, varying font sizes, etc.); interaction provided by software; program user friendliness and learning environment; and, record keeping

2. In a general way, how is culture represented and used in the program?

3. Does the software provide contextual language use and practice and develop skills that can be transferred to situations outside of the classroom?

Chapter II provides the background behind today's ESL classroom which is needed to better understand the requirements of CALL material and the evaluation of that material. Also presented is an overview of CALL shortcomings and potentials, as well as design considerations for the creation of today's CALL materials. Chapter III describes the CD-ROM selection criteria, the formation of the evaluation instrument, and the methodology of the study. The study's limitations are also found in this chapter. Chapter IV presents the findings from the evaluation; a matrix is available for quick reference and an objective descriptive summary and subjective critique highlighting strengths and weaknesses are available for each program. Chapter V discusses the findings in reference to the research questions. Conclusions are then drawn and recommendations for future work are given. 


\section{Definitions}

Since the study is on a computer-based topic a number of technological terms have been used throughout the paper. Extreme "technolect" characterizing today's computer industry has been kept to a minimum; the following supply the definitions of those terms possibly unfamiliar to the reader. Some ESL-specific terms are also included.

Algorithms - Repeating finite mathematical instructions used for the decoding of language into a structure recognizable to a computer.

$C A L L$ - Computer Aided Language Learning. The use of computers and associated software material as a medium of practice and instruction.

$C D-R O M$ - (Compact disc read only memory). A disc containing large amounts of digital data recognized and interpreted by a computer.

Communicative Language Teaching - The most current approach to language teaching in ESL where the ultimate goal is the teaching of language and language learning strategies for communicative language use away from the classroom (in comparison to other approaches that have focused primarily on the teaching of grammar).

Cyberspace - A virtual space provided by computers and computer programs that can be navigated and experienced by the user. 
Developing - Conceptualizing and creating a computer software application.

(Programming and developing are used interchangeably in the paper).

Digital - Referring to information that has been broken down into numbers in order to be handled by the computer.

DVD - (Digital video disc). Read-only memory like a CD-ROM having the same physical characteristics but a larger storage capacity (seven times that of a CD-ROM).

Fossilization - When a learner can no longer reach a level of linguistic correctness because of the prolonged internalization of the incorrect linguistic form.

Hypermedia - Embedded multimedia links that allow for user-defined branching (for example, the clicking on a photo may take the viewer to associated video footage, or a textual description).

Hypertechnology - Embedded links in multimedia applications that allow for user defined branching of information in a non-linear manner.

Instructional Design - The pedagogical structuring of information/material in an easily accessed way that will promote learning. Today's Instructional Designer for computer material is called an Information Designer, who not only is in charge of the structuring of information, but also makes sure that the media used for the content make sense and that the interaction and environment created by the software make sense to the user. 
Interface - A graphical overlay (usually metaphorically based) that allows the user to interact with the computer.

Multimedia - The use of two or more media in an integrated computer application.

Operating System - A software interface between the computer's core abilities and the user. A DOS-based operating system is an early computer operating system made by Microsoft in the 1980's.

Programming - The writing of the code that makes the computer software deliver the features of the program. (Programming and developing are used interchangeably in the paper).

Share-ware - Software that can legally be shared in exchange for a small fee (usually $\$ 5.00$ to $\$ 35.00$ ).

Surface Features - The aesthetic features on screen displays/interfaces (i.e. color, borders, font styles).

User - An individual interacting with a computer. 


\section{CHAPTER II}

\section{REVIEW OF THE LITERATURE}

This chapter looks first at current teaching practices and at how existing methodology and materials support state of the art ESL. Then, the role of technology in ESL is discussed, priming the entrance for the latest technology: the computer. A brief background on computer instruction is presented, followed by a current assessment of CALL for today's ESL classrooms and students. Instructional design issues relevant to computers as a medium of instruction are introduced with a look at how material designers might provide for these issues. The opportunity for the representation of culture in CALL materials is also discussed.

\section{Today's ESL Classroom}

In order to better understand today's ESL environment in which CALL is appearing, this review of the literature will first present an overview of the teaching approach and methodology characterizing current classrooms. By understanding the basic needs of state of the art ESL and the underlying theories of language use and language learning, one can begin to make an adequate assessment of the materials and practices required by such an environment. The needs of current ESL students should be reflected in the materials and practices incorporated into the classroom. The 
materials used in a classroom are chosen for their ability to meet classroom demands, and, by extension, chosen to support the current style of teaching.

\section{Communicative Approach}

The history of TESOL has been dotted with numerous approaches to teaching. The latest approach adapted by TESOL educators is the communicative approach. This approach to teaching English evolved from work done by anthropological linguists who saw language as "first and foremost...a system for communication" (Celce-Murcia, 1991, p. 8). Hymes (1974) contributed much to the communicative paradigm. He recognized that a theory of language must not individually examine parts (such as grammar and culture) in isolation. Instead, those parts must be considered as mutually functioning components of speech; one part does not exist without the other. With Hymes' work helping to lay a theoretical foundation, language teachers began to regard their language classroom and associated teaching methodology as tools with which to promote language learning. The main goal became communicative language use.

A communicative classroom is distinguished from other classrooms in many ways. For instance, as stated above, since language is seen primarily as communicative in nature, the communicative classroom will encourage communicative tasks (see Conrad, 1996; Mohan, 1992; Nunan, 1993; Savignon, 1993). Teachers and the communicative tasks they ask of their students "[encourage the] students to ask for information, to seek clarification, to use circumlocution and whatever other linguistic and nonlinguistic resources they [can] muster to negotiate meaning" (Savignon, 1993, p. 38). 
$\underline{\text { Language Learning Strategies }}$

By teaching language learning strategies a student's proficiency in the language can be further enhanced. This can bolster student self-confidence (Oxford, 1990). With a teacher's guidance students are able to recognize a learning path that best fits individual student needs. Oxford relates how "learning strategies help learners participate actively in...authentic communication" $(1990$, p. 8). Specific communicative strategies will help with the development of a sociolinguistic knowledge and competence. For example, students can learn to ask questions (social strategies) or learn how to compensate when linguistic meaning is unknown (compensation strategies). In the communicative classroom students are encouraged to learn how to become autonomous learners.

Self-direction is a key component in today's ESL classroom. The teacher will not always be with the students to help them learn and to successfully communicate. Therefore students must take the responsibility to learn for themselves. Rivers (1976) asserts that students should strive for autonomy in interactions and learn to rely on their own abilities, at an individual level, away from the support of the language classroom. She is an advocate of teaching communicative strategies that ultimately give learners the power to use language outside of the class as they encounter relevant and meaningful situations. Dubin and Olshtain (1986) also refer to students' need to acquire "learner autonomy"(p. 102). They assert that it is not what a student can do with a language that is paramount, instead, what needs to be taught in the classroom is how the student can learn to become an independent language learner. Littlewood (1981) states "since the relationship between form and functions is variable and cannot be definitely predicted outside specific situations, the learner must also be given opportunities to develop strategies for interpreting language in actual use" (p.

3). Because classroom activities will guide the choice of strategies used by students 
(for example, distinct grammar learning will produce analysis reasoning-type strategies; Oxford, 1990), activities that promote more global communicative strategies must be offered.

\section{$\underline{\text { Language Use }}$}

The functional view of language incorporates both pragmatics and language use, along with the structural view of language composed of the linguistic forms. A mastery of linguistic structure (grammar) is often not enough to enable a student to competently communicate in real-world situations; knowing how to conjugate verbs does not ensure success when out on the street. Therefore, the functions of language (such as making requests, using apologies, using refusals) are taught to students. However, even learning the "semantic formulas" (Cohen, 1996, p. 254) does not mean one knows how to use them appropriately.

To better prepare students, sociocultural information along with the linguistic forms needs to be included in instruction. In this way, students not only learn the patterns of speech found in speech acts used as language functions, they also can learn the underlying cultural information dictating the use of one speech act over another. This technique allows the student to reach a better understanding of the social meaning behind the linguistic forms (Littlewood, 1981). By understanding the need for teaching both the functions and the structure of language, ESL teachers prepare students for communicative use of language outside of the class. In a communicative classroom, no one language aspect is emphasized more than another. Instead, establishing the relationship between form, meaning, and pragmatic use becomes a standard practice (Larsen-Freeman, 1991). Activities for learning language use include speech act practice in model dialogues, role playing, and situation evaluation. 
$\underline{\text { Feedback }}$

Feedback becomes a crucial component in a communicative classroom because students gauge their linguistic and sociolinguistic output during practice by how successful they were with the task (Littlewood, 1981). Crookes and Chaudron (1991) support the use of materials and activities in the communicative class that "provide opportunities for learners to recognize the communicative effectiveness of their target language productions" (p. 61). H. D. Brown (1994) discusses two major types of feedback: affective and cognitive. Affective feedback is relayed through kinesic behavior. Oculesics, facial expressions, and tones of voice are examples of affective feedback. Such behavior can provide emotional affirmation of the participants in a speech exchange. Cognitive feedback according to Brown is usually linguistically relayed. For example, one participant may tell or signal to the other participant that the message was not understood. The participant will then be made cognitively aware of their performance from the feedback of another.

Brown states that above all, positive affective feedback is required. Without the affirmation of others during an interaction, learners may see no reason to attempt communication. If a person affectively feels accepted, the negative cognitive feedback received is less likely to discourage future communication. Positive cognitive feedback will provide the students with reinforcement that their utterance was accepted and correct. A misuse of positive cognitive feedback (for example, leading the learner to believe that their ill-formed utterance was correct) can have debilitating effects on language learning. Larsen-Freeman and Long (1991) point out that if a student is not made aware of consistently ill-formed language, fossilization may set in and render future correction impossible. 
Treatment of Errors

Error analysis and treatment of errors as a form of feedback need to be greatly considered in today's ESL classroom. Without a focus on form, the question of where and when to correct during functional language use must be addressed. H. D. Brown (1994) declares that if too much emphasis is put on errors, the goal of communicative fluency is not being properly considered. Findings in second language acquisition studies are providing more and more clues to the sources of student errors. Some errors can be attributed to a common developmental sequence (Crookes \& Chaudron, 1991; Larsen-Freeman \& Long, 1991); others may be due to first language (L1) interference (Larsen-Freeman \& Long, 1991). Still others may be only a one-time mistake and not an error at all (errors reflect the student's linguistic mastery of an item and mistakes signify a performance lapse; Larsen-Freeman \& Long, 1991). Understanding more and more about the significance of errors (i.e. not all errors are bad) creates a need for students to be given the opportunity for the experimental use of language. The teacher's role is to guide the learner in becoming aware of errors and mistakes and the possible reasons why the errors are being made, but not to focus on them. Grammatical corrections are still provided, but they are not the primary focus of a communicative classroom.

\section{Student Affective Factors}

The feedback students receive should not only help them with their progress, but also encourage students' language use and provide motivation for further communicative development. As an affective factor in language learning, motivation has a powerful influence on the learner. H. D. Brown (1994) relates that "the most powerful dimension of the whole motivation construct in general is the degree to which learners are intrinsically or extrinsically motivated to succeed in a task" (p. 155 
- author's italics). Intrinsic motivation refers to there being no visible reward for doing an activity; in other words people complete a task for the sake of completing it (a personal satisfaction is the reward). In contrast, extrinsic motivation relies on a perceived value or reward that follows the completion of a task. In this case the gain may be an increase in pay at a job, a higher grade or admission into a better university program.

In language learning, as well as in life tasks, intrinsic motivation is held to be superior to extrinsic motivation (Brown, H. D., 1994). In the ESL classroom students will bring different degrees and different types of motivation into the classroom. To address the end goal of teaching students how to successfully communicate, instructors must find ways of motivating students. Selecting topics useful and relevant to students' lives is one way to encourage and maintain motivation, according to Rivers (1976).

Besides motivation, other affective factors like attitude toward learning, risktaking, and anxiety have a large effect on successful language learning. Seen as defenses learners place around themselves, the build up of emotions is referred to as the affective filter (Krashen \& Terrell, 1983). The filter is a psychological construct. If the filter is too high, students are less receptive to learning. If the filter is lowered, learning can more easily take place. Because of the pressure students may feel when learning a language (and an even greater pressure to communicate successfully) the classroom should provide a comfortable atmosphere for learning. The humanistic atmosphere of today's ESL class (e.g. focus on the learner, concern with student affective variables) provides a supportive environment to promote language learning and a nurturing environment so that students can feel at ease.

This new-found focus on the learner replaces the teacher focus found in earlier teaching approaches. The communicative classroom is not a teacher-fronted domain; 
"teachers...need to remain aware that they are not in the classroom to fill up time with the sound of their own voices" (Crookes \& Chaudron, 1991, p. 47). In a learnercentered classroom error correction is minimal, students are not put on the spot to perform, and the learning of material is through collaborative activities taking place in small groups. Within a context that appears to be more social instead of academic, students are left to negotiate meaning and to focus their attention on meaning instead of form. Affectively this promotes a more relaxed platform for learning to take place (Stevens, 1993). According to Celce-Murcia (1993), there is "evidence that 'communicative' language classrooms - especially those that preclude any learner focus on form - produce better language learners than do traditional classrooms" ( $p$. 291).

\section{$\underline{\text { Student Cognitive Factors }}$}

Student learning styles are another relevant feature of today's ESL communicative classrooms. Learning styles are different from language learning strategies in that strategies are used in the acquisition of strategic competence for successful interaction away from the classroom. Learning styles are the different modes of how people prefer to learn. In an ESL setting it is not uncommon to find a heterogeneous assortment of cultures. As members of specific cultures, students not only have developed culturally specific ways of learning, but they also have their own individual styles and preferences. Even in a culturally homogenous classroom there will be numerous individual differences. ESL instructors must then provide for the wide variety of learning styles through various tasks, subjects of study, and materials (Scarcella, 1990):

Categories of learning styles are based on the main modes of learning: aurally, visually, or through tactile means. Also, preferences for group or individual learning 
styles or what task types students enjoy and are used to (e.g. rote grammar or communicative activities) are considered. The teacher, aware of cultural and individual differences, needs to be able to recognize the learning styles manifest in the group of students. Then the teacher can provide instruction and "teach in the ways in which students learn" (Peck, 1991, p. 364). Although individualized instruction in large class settings sounds unrealistic, the use of varied activities and materials can again provide an assortment of input to satisfy individual needs.

\section{Importance of Input}

The varied input provided to ESL students, used to meet individual learning needs, must also be comprehensible. Comprehensible input refers to language that is understood by the learner. As such, the learner is given the freedom to focus on the meaning of the message since the linguistic structures are already understood. Swain (1985), however, concludes from a study that comprehensible input does not alone ensure native like production of student output. He concludes that input requires an environment made up of interactions based on the negotiation of meaning. Through the use of activities using conversational turns, students can receive input from one another, receive feedback, and provide output for authentic language use. This type of practice allows students the opportunity to test their sociolinguistic knowledge by referring to what they already know. Students are even given the opportunity to venture new ways of expressing themselves. Referring to the output as "comprehensible output" (1985, p. 249), Swain relates the need for students to be given the chance to try out language so that they can come up with their own messages and intended meanings.

Pica and Doughty (1985) emphasize the importance of input and interaction in language learning. They relate how many materials in ESL do not fully account for 
the two-way exchange of information needed for student language growth. Their study looked at the nature of input and interaction between a teacher fronted class and compared it to the input and interaction of small group activities. Pica and Doughty found that teacher-fronted activities provided more features of negotiated comprehension than did the group work, although there was more target language production done by the students working in small groups. They conclude that pairwork will provide students with a better opportunity for interaction and the negotiation of meaning.

\section{Materials and Activities}

Activities for the ESL class, besides being varied, must provide for conversational interactions that reflect realistic language use. These interactions will provide the opportunity for both comprehensible input and output as students communicate with one-another using meaningful and realistic language. Beyond the provision of routinized interaction, students must also be given the chance to use their strategic competence. For example, Savignon (1983) suggests activities where students find themselves coping with unexpected incidents. Although such activities could potentially promote student anxiety, the pleasant classroom environment should counter act debilitative feelings.

Besides the use of activities for real-world communicative exchanges, materials need to be just as realistic. Authentic items, those not created for ESL educational settings, have become a staple in the ESL classroom. The use of actual menus or bus schedules contributes an authenticity to the language practice taking place. Purgason (1991) offers some tips on lesson planning that include the use of authentic material to support authentic language use. Her other considerations for classroom planning go beyond materials and refer to the necessity of teaching only 
what the students need to do with the language in the outside world; minimal provision of sentence level discourse and not one-word responses; informing students of lesson objectives; use of clear instructions for all activities; provision of feedback that is relevant and useful to the task at hand; provision of real-world activities that encourage student participation and interaction through the negotiation of meaning.

\section{Culture Learning}

As mentioned above, the ESL classroom as a setting reflects the cultures of the students found within it. Even if the class has a homogenous group of students, their contact with a foreign language brings them face to face with underlying cultural assumptions - their own, as well as those of the foreign language. These assumptions will manifest themselves through language use. Yalden (1983) points out how the communicative shift in the classroom can act as an empowering tool; students are given a means to become members of the speech community. Even though they may not want to become full members of the speech community, at least students in the communicative classroom are given the opportunity to learn about others and about themselves as bearers of culture. Cultural and intercultural learning create a fifth dimension in the ESL class (the other four dimensions are speaking, reading, listening, and writing). Access to culture and culture learning needs to be integrated into classroom materials so that students can at the very minimum have access to the underlying culture of the language being studied.

Damen (1987) proposes that "learning how to learn about a new culture is the primary skill needed for effective intercultural communication" (p. 53). As a functioning component of communicative competence, culture has an active role. Damen reflects on the need for teachers, who are usually members of the target language culture, to teach students about patterns of culture; it is assumed that the 
instructor's first hand knowledge of a culture can provide competent interpretations for classroom use. Byram (1988) adds that the contributions a foreign language teacher can make, just by their having a different cultural orientation or an "otherness" ( $p$. 20), can be a significant addition to culture learning in the classroom.

To become successful culture learners, Damen suggests that teachers need to first become explorers of culture. Drawing from anthropological fieldwork, Damen proposes that teachers become familiar with the culture of another by using techniques such as contrastive analysis, and the formation of cultural hypotheses. Contrastive analysis concerns the interpretation of cultural behavior in contrast with another's cultural behavior. It is crucial that the interpretation does not become a description or comparison that sets one culture against another. Then, the formation of hypotheses allows the refinement of the cultural interpretations. As a component of the basic scientific method, a hypothesis will be modified in light of new evidence; in the language classroom culture hypotheses can then be modified or accepted based on observed behavior. Once instructors have reached an awareness of how to become cultural learners, their knowledge can be passed along to students.

Hoopes (1979) characterizes intercultural learning as taking place along a continuum. At the one end is ethnocentrism. Here the operative principle is a feeling of superiority over another, either culturally or personally. At the opposite end of the continuum is a much higher level of awareness and acceptance of others. Here the learner has achieved a state of understanding of the differences found cross-culturally. In between each end of the continuum are levels the learner passes through that continue to raise the learner's degree of awareness of other cultures. Slowly the learner begins to shape an understanding of, a tolerance of, and an appreciation for cultural difference. Students learn how to suspend judgment of behaviors by learning not to use their own culturally based system to judge another's. 
Taylor (1994) refers to the process of becoming interculturally competent as a transformation. This transformation affects the cognitive, affective, and behavioral dimensions of the student. Intuitively, changing behavior is much easier than changing the emotions acting as the source of the behavior. Once the cognitive dimension of making the student aware of feelings and behaviors is activated, the student must be given a comfortable environment in which familiar feelings can meet with unfamiliar examinations of culture. Kramsch (1993) suggests a neutral zone that can be used for the examination of culture. In it "the expressions of meanings [are not held] hostage to meanings of either their own or [the] target speech community" (p. 14). This neutral zone acts as a third place where the student's own culture and the newer target language culture can mingle together. In this third place the student learns to adapt to cultural situations, being aware of how culture will affect feelings, thought, and behavior. Today's ESL environment can provide the neutral zone referred to by Kramsch. In the classroom the use of activities that promote a cultural awareness will greatly benefit the intercultural ESL learner. Tomalin and Stempleski (1993) offer an assortment of activities meant to make the learner aware of how culture globally and individually affects behavior. The exercises Tomalin and Stempleski present provide opportunities for students to gather information, discuss their findings, and then interpret what they have found in light of their own culture as well as the one being examined.

Without the recognition of culture as a catalyst of successful communication, and without the examination of culture, the ESL learner can not begin the process of gaining intercultural competence. Language learning goes beyond linguistic codes. It encompasses an awareness of both language and culture. Byram (1988) concludes:

If foreign language teaching is carried out as an integration of all four areas of experience (language learning, language awareness, cultural awareness, and 
cultural experience), can it fulfill the responsibility not only of creating users of other language codes but more importantly of educating them by introducing them to foreign language and cultures and thereby re-introducing them to their own and to themselves. (p. 30)

Besides cultural differences, differences through the enactment of gender and gender roles are also a consideration in materials. "Sex role stereotyping exists in both the content of educational materials and in the classroom interaction patterns" (Cooper, 1993, p. 123). Cooper looked at illustrations in school textbooks and found that many more males were pictured then females. When women were pictured, the roles they were in supported traditional sex-role stereotyping: women were shown in less dominant roles. The treatment of gender in activities and materials in an ESL classroom can benefit from the theory base of culture learning. Cooper stresses that student perceptions will be affected by gender stereotypes; students will interpret the behavior of others based on stereotypes, and then store the interpretations as data for future inferences. While every culture has its own gender roles, it is important to maintain a positive and neutral classroom environment so that intentional or unintentional negativity towards classmates because of one's interpretation of gender is not perpetuated.

Overall ESL educators have a commitment to students to wisely incorporate the world outside the classroom inside through existing methodology. Technology has provided a tool that has a potential classroom application. The computer, as a medium or instruction, is showing up more and more in ESL. Its appearance follows a fairly established history of technological innovations adapted for educational use. 
Technology as a Medium of Instruction

\section{Traditional Technology}

The history of the theory and methods of language teaching has to some degree followed technological advances: ink and paper replaced oral techniques of language learning found in the Pre-Renaissance and changed the focus of learning to linguistic form, not use. The printing press in the 15th Century allowed a mass production of text and the formal study of grammars (the initial Grammar-Translation method) began. The breakthrough of the tape recorder allowed the methodologists to followed suit with the Audio Lingual Method (Celce-Murcia, 1991). The point is that there are and have been visible connections between technology, language, and language instruction. "It is accurate to state that instruction methodology has evolved in concert with media technology" (Layne \& Lepeintre, 1996, p. 228).

Before the computer, technology found its place in the classroom through video, film, filmstrips, cassettes, overhead projectors and the like. This assortment of technical media was incorporated into existing classroom methodology because it could support various learning objectives and classroom needs. In the ESL classroom, the educational technologies through various media are used to bring the outside world into the classroom. Media were also used because they provided motivation by presenting language in a more complete communicative context (Brinton, 1991). For example, a video or film can show students examples of language use; cultural slide shows can present dynamic settings for discussions; video-taping can give students a more astute awareness of their linguistic performance. However the media are used, the purpose for their inclusion is decided beforehand, with careful consideration as to which medium is selected over another. Brinton explains that "ultimately each 
medium leaves its own imprint on the teaching/learning process, and it is up to the teacher to decide which one to select in order to teach a given point" $(1991$, p. 458).

Brinton details some issues associated with media. Of main importance for any media, technical or not (i.e. electric generated objects versus non-electric), is its appropriateness for the learning audience. Technical quality must also be considered, and as mentioned above, how to incorporate the media into the classroom methodology needs careful planning. Outlining the rationale of why media might be used in a language classroom Brinton declares, "given the role media play in the world outside the classroom, students expect to find it inside the classroom as well" (1991, p. 456).

\section{The Computer}

Outside of the classroom computer use can be enormous. The growing number of services offered on the World Wide Web has allowed global contact without leaving the house; doctors perform practice surgeries without a patient; pilots practice flight maneuvers on million dollar aircraft without leaving the ground. Computer use has become a recognized and accepted practice both in and outside of the classroom. The computer's role in an ESL setting is receiving more and more recognition for as technology offers greater alternatives to what the computer can accomplish, its incorporation into existing methodology becomes much more realistic and practical for today's teaching approach.

Hypertechnology and Interactive Multimedia

Before the development of hypertechnology, a linking system for information, computer programs were linear in design, and supported the behaviorist paradigm of learning. Progression was from a sequencing of screen to screen and activity was 
slow. Mohan (1992) claims that these early programs were basically a dumping ground for traditional workbook materials because reworking the workbook into software form was easy and cheap for designers to do. As a result, computer activities took on a striking semblance to workbook activities. Fill in the blanks, cloze activities, multiple choice reading comprehension, vocabulary practice, text reconstruction, and close-ended questions were typical of the early behaviorist programs. These programs were mechanical, unstimulating behaviorist models with a lot of rote drills and "wrong, try again" responses (Mohan, 1992). Such rote learning practices appeared to many as lacking inherent relevancy to what the student was learning. As theories of language learning were developing beyond behaviorist models, CALL remained locked in the behaviorist paradigm. The creation of hypertechnology brought a more dynamic alternative for software programming.

With the use of hyper-technology, especially with Apple Computer's "Hypercard" released for use in 1987 (Ambron \& Hooper, 1990), the programming for educational and ESL products took on a new dimension. While multimedia were already being used in the classroom (e. g. video, film, filmstrips, cassettes, overhead projectors), a tool like Apple's Hypercard primed the way for an interactive multimedia stage. Hyper-technology together with the increased storage capabilities and the innovative graphics accessories could put some life into the old linear behaviorist programs. The results are multimedia programs that are dynamic in nature, can be visually appealing, and are fun to use.

The links provide a non-linear way of viewing/using the information; a student is able to go in different directions to access related information in an exploratory manner (Harland, 1991; Sussex, 1996). The hyperlinks provide students with an opportunity to look for and create their own meaning in learning. Programs with hypertext have been found to keep students' attention and provide opportunities for 
good language output (Conrad, 1996). Chan and Liu (1992) propose the use of hypermedia in the linking of information into a "semantic network" (p. 50). Because language students do not learn in a linear fashion (Crookes \& Chaudron, 1991), nor do they partake in similar learning routes (Larsen-Freeman \& Long, 1991; Lian, 1992), Chan and Liu's project of a digitized interactive text provides a more learner-centered environment. Learners can explore and find the paths that best suit their needs. The technology inherent in hypermedia produces a new form of communication. Chan and Liu explain, "for several centuries, the communication technology of the book has defined what is meant by foreign language education, and its linear, static, and silent constraints have long directed the development of many teaching and learning methods" (1992, p. 49). Hypermedia complement the empirical findings of how people learn; the timeliness of the technology provides an opportunity to continue experimenting with how people learn. Such knowledge can then be transferred to the design of pedagogical software.

\section{Interactivity and Emerging Paradigms}

The dynamics provided by the program are gauged by the amount of interactivity the program provides. Some might say that by its very nature a computer promotes interactivity (Cooks \& Henstock, 1993). The computer may, in a basic way provide a sort of interactivity, for one must interact with the computer to accomplish any sort of computer-based tasks. However, the extent of the interaction in computer programs must be considered. The simple clicking on objects or on "Y"/" $\mathrm{N}$ " responses does not show the true possibilities of interactivity. This sort of interaction certainly doesn't provide any significant communicative language use. Jenise Rowekamp of the University of Minnesota is also concerned with the meaning of interactivity and how best to categorize different types of computer interactivity 
(personal communication, March 5, 1997). She relates how interactivity has associations with everything from the basic drill and practice activities to simulations.

Simulations are programs where students actively participate in the outcome of a story, situation, or game. In a simulation, the context changes through the interaction and the choices students make. Unlike drill and practice activities where answers can be quickly relegated to correct or incorrect, simulations have no right or wrong answers (Schreck \& Schreck, 1991); instead, students learn problem solving techniques and are left with the consequences of their actions as the program progresses. A simulation, then, provides an example of exceptional interactivity. Coleman (1991) defines true interactivity as "a dialogue in which both sides of a twoway exchange adapt their behavior in the light of the other's response" (p. 93).

Multimedia programming using interactive media can be categorized under three basic paradigms: the environment, the tutorial, and the database (Blum, 1995). Simulations might best fit under the environment paradigm. Environments create quasi-virtual worlds where the user can explore a realistically rendered three dimensional atmosphere. Brøderbund's Myst game is an example of an environment. In Myst the user can open hidden doors, walk down hallways, and search for clues to the island's mystery. The graphics create a realistic atmosphere where users can feel as if they are actually taking part in the actions.

Tutorials are based on the teaching of specific objectives. They are programs that are linear in design (that is, they are programmed to not allow the learner extensive exploration of the program), with usually one main learning path established for all learners. Progress measurement features are found in tutorials so that users can gauge their skills and knowledge against the predetermined outcome (learning objectives) of the program. Schreck and Schreck (1991) remark that a better produced tutorial can provide a great deal of interactivity through answer and feedback 
provisions. Tutorials work well for individualized learning; a good tutorial program will include levels of difficulty and other customizable features.

The database paradigm is built on the structuring of information into segments that can be easily searched, located, and retrieved. The use of an interface will provide the link between the user and the wealth of stored information. Interactivity in database paradigms is behaviorally based; interactivity is characterized by the clicking of buttons to retrieve the data so that information can be accessed. Microsoft's Encarta is a good example of a non-ESL database application available commercially. Examples of ESL programs in CD-ROM database paradigms are multimedia picture dictionaries, versions of popular games, and simple stories in text or audio.

The type of paradigm a program is categorized under will consequentially determine the type of interactivity. In many programs there is a combination of paradigms (Blum, 1995). As such, there is a range of interactive potential for any one program. Cooks and Henstock (1993) agree that simply clicking on objects is a minimal type of interaction. They relate that the benefits one receives from such a program are pedagogically low. In the development of computer activity templates that go beyond the simple click routine, Cooks and Henstock require the responses to the activities be typed into the computer so that the typing of the response might reinforce the learning taking place. A simulation, however, will provide the best opportunity for realistic interactivity. Databases provide basically mechanized interaction, and tutorials, although providing contextualized practice, have a predetermined path. Schreck and Schreck (1991) again offer an opinion: they believe that future tutorials should be better able to replicate communication through the provision of question-answer formats that enable a running dialogue between the user and computer, using linguistic information drawn from a large database of language. 
Reliance on Visuals

One of the more appealing aspects of multimedia is the potential for visual representation in learning. The consumption of images and imagery keeps growing at a steady rate (Hooper, 1990). As a result, many people now expect to see visual representations with color, and a fluidity between text and graphics (Nanny, 1990). With the use of television resulting in a steady decline in reading (Belch \& Belch, 1995), a visually stimulated audience outside and inside the classroom is being molded. The use of multimedia can, therefore, maintain attention by providing the visual stimulation people are becoming more used to. Although many ESL students may not have the same tendency toward television watching as mainstream USA nationals, the visual quality of the programs can be motivational factors for students as they work on second language learning through CALL.

Multimedia programs by themselves rely on a great deal of visual imagery to communicate with the user. According to Lee (1996) "people throughout the ages have relied on different forms of visualization in an attempt to improve communication" (p. 58). The digital environment has produced a new type of functional imagery in the form of icons. Most computer program interfaces depend on the use of these symbols to represent functions of the program. Most often a program will be metaphorically based, allowing the user a familiar context within which to navigate and work. For example, a trash bin on the screen can be recognized as where deleted files will be taken; a file cabinet with folders can guide the user to the program's filing system. Good icon design can facilitate a pleasant experience, if icons are intuitively discernible. A poor design with inappropriate or unrelated icons, or inconsistent use, can be frustrating (Lee, 1996).

In an ESL environment icons need to provide a relevancy that can reach many cultures. Culture-neutral icons are likely rare to come by. Instead, the use of touch 
design can provide textual information to let the user know the icon's function. Touch design programming positions bubbles of text next to the icon whenever the cursor passes over the icon. With repeated exposure and familiarity people will interact faster and be more comfortable with the program's icons (Lee, 1996). However, considerations need to be made for first time users, especially ESL learners. With those learners in mind the use of touch design for any icons would be helpful if the students are unfamiliar with the computer and associated iconography.

The combination of various media into a single package has also led to an emerging field: media psychology (Luskin, 1996). Media psychology relates to how the mind experiences and responds to the multi-sensory environment inherent in today's computer technology. The combination of media can indeed provide multiple channels through which individual learning styles can be satisfied (Guglielmino, 1991; Brinton, 1991). Yet the relevance of the media and the choices of one medium's inclusion over another needs to be studied because students will respond differently to the various media.

\section{Computers in the Communicative Classroom}

CD-ROMs allow the combination of media into a single educational package. The data storage capabilities of CD-ROMs also facilitate what might be considered the most important digitized element for the ESL classroom: video. Video can act as an excellent source of cultural input by providing examples of the contextual use of language in a cultural setting (Coleman, 1991; Ito, 1996; Kenning \& Kenning, 1990). Sociocultural rules of language use presented through digitized video can be accessed up-close, providing students with a front-view seat to how language can work as a 
means of communication. Speech acts and their varied sociolinguistic forms can be made available and students can see the context of language that often helps with comprehension (Ito, 1996). Besides the linguistic representation of language, students can also see how kinesics function in communication. Facial expressions, gestures, and spatial positioning are but a few of the paralinguistic representations that natives respond to almost instinctually, but that pose one of the hardest areas in human behavior to learn, or even recognize (Chaika, 1989). Overall, multimedia, especially through its use of video, has the potential of lending a multi-sensory, stimulating, and linguistically rich environment that supports language learning.

\section{$\underline{\text { Language Skills }}$}

The majority of today's software offers the educator and user a range of activities in three of the four general language skills: reading, writing, and listening. The skill of speaking is left out. Skeptics of CALL are first to react and say that the main goal of the communicative approach is to teach oral communication. However, some might say the verbal communication between a human and computer does not even make sense because the computer does not care; there are no affective feelings as in authentic communication. Oral communication practice in the ESL classroom when students are one-on-one with the computer is an area in CALL that needs some developing.

The biggest problem for oral communication is that technology cannot yet fully synthesize spoken language and all of its variant properties. Higgins and Johns (1984) assert that the "problem for a speech synthesis program is not the number of variations but the way in which each variation is conditioned by the context in which it appears and by the intention of the speaker" (p. 31). Humans can decode the varying sound waves of many different speakers who use great amounts of phonetic variation 
during speaking, but the computer can not. Higgins and Johns also assert that until there is a computer that has the same thoughts, feelings, and actions as a human, complete speech recognition will never be available; until a model of human memory is developed by fully understanding the physiological processing of language, complete natural language use with the computer is not an option.

Besides not being able to parse the spoken word, the computer is also unable to parse the written language. Although a syntax of a language strives to create a finite set of rules for a language's grammar, there is an infinite number of combinations once lexical items are added to the syntactic formulas. As such, a computer can only recognize what the database has stored, or rely on algorithms that decode simple and restricted language. Because the computer is unable to recognize complex speech and writing, today's technology cannot fully diagnose errors that occur in the free flow of conversation (Conrad, 1996; Kenning \& Kenning, 1990).

Although technology has not supplied the means to accurately parse nondeterminant conversational strings, Brierley (1991) describes a project based on a computer program known as $E L I Z A$ that uses natural language processing, but only in restricted domains of language. Brierley says that besides not allowing for meaningful and relevant communication outside the restricted environment, the computer, as a participant in communication, lacks any affective characteristics and so is not interested in user input anyway. Conrad (1996) suggests that as artificial intelligence advances in the computer field, CALL will one day be representative of communication found between humans. As long as this technology is not yet available, one must make do with what is available for use in the communicative language classroom.

Speech, though one of the main forms of communication, is not the only form. The written word also acts as an arbiter of communication, and computers can deliver 
this language skill with much support. Word processing programs are actively used for students' writing in both ESL and non-ESL environments. Word processing offers the student an effective way to check spelling, grammar, and make rewrites for classroom assignment. This skill is useful in and outside of the ESL classroom and can be easily incorporated into daily lessons or homework as long as the computers are available.

In terms of actual communication, the largest facilitator of computer communication is through the Internet. Through the Internet businesses, organizations, and individuals can and do communicate using e-mail. Some people, however, wonder about the integrity of the communication taking place, and they have a fear of losing the meaning of the message because of the preoccupation with the medium of the computer (Tuman, 1996). Meaning soon becomes relevant in these situations because the users, through repeated exposure, have moved past the fascination of the medium and on to its utilitarian use. The use of a network, either globally based like the Internet, or situationally based (i.e. found only in the school or university) provides channels where on-line written communication can occur. One study found that when students exchanged meaningful messages with other students via a network, attitudes were favorable and participation was more than it had ever been (Conrad, 1996). By allowing for meaningful interaction, even in an alternate contextual setting as cyberspace (versus face-to-face), ESL learners are given a motivational tool to use.

Interaction between people done through the medium of computers can often lose the human feel of communicating, even though exchanges of information can be just as meaningful. Kenning and Kenning (1990, p. 63) remark that, for some people, "to replace one of the participants in an exchange by a machine is a dramatic change." The computer to date has done nothing to deplete the quality of communication that 
transpires from its use, especially since both print and the telephone have already made people accustomed to communication in non-proximity. Patience, guidance, and a positive attitude of the instructor, as well as consideration from the programmers who need to develop more user-friendly software, can overcome a cold technological feeling.

\section{Collaboration with Computers}

If the computer can not be used as an individual verbal communicator with one student, the computer does act as talk elicitor when a group of students are at the computer. As mentioned earlier, cooperative group work is a major component of a communicative classroom. Through computer use this practice can also be replicated. The use of games with groups of students at a single computer provides an atmosphere of collaboration and negotiation of meaning to decide each move (Cheung \& Harrison, 1992; Conrad, 1996; Kenning \& Kenning, 1990), and fun. Mohan (1992) stresses that any software requiring problem solving will result in more oral interaction when students are in a group at the computer.

Renié and Chanier (1995) based their computer program, ELEONORE, on collaborative learning. The goal of ELEONORE is to have students, either in a group, or working alone, think of the computer as a collaborator that helps in reaching an understanding of a complex grammatical structure. Through the provision of grammar as well as the sociolinguistic background of the structure, students are able to improve their communicative understanding of when and how to use the structure. Renié and Chanier assert that the computer has a synonymous role to the human collaborator; the computer acts as an expert who has a superior knowledge of specific information that will be passed along to the student who initiates the interaction. In this sense, the computer acts as a neutral companion, who guides the students instead of judging 
them. As a companion the computer shares its knowledge with the students. Even feedback will let students be aware of whether the error was sociolinguistically or grammatically based. The ELEONORE program allows students to develop a sociolinguistic competence through a mutual collaboration with the computer; students must solicit information from the computer, as in normal communication, and the computer responds accordingly.

Communicative speaking skills and tasks can still be developed through the medium of the computer. High quality audio has made the computer an excellent language conveyor. Students can hear natural language use, and with common software recording features can record their own voices and hear the results instantly. Since intonation in language learning can be very difficult for some students to recognize and hear, a computer program can slow down the audio, or offer graphic representations showing pitch raising and lowering (Higgins \& Johns, 1984). Aural databases acting as dictionaries can also provide students with samples of authentic language use.

Borras (1993) developed a program, Practicing Spoken French, for students to improve oral communication skills. After first viewing a video segment, students then answer comprehension questions. Next the students draft a description of what they saw by answering descriptive questions (if students need to access the video again they can). After the draft is printed out, students record their descriptions that the instructor will later access. Borras' program provides the learner with authentic material to use ultimately in a speaking exercise. She creates speaking practice for a specific language task (i.e. giving descriptions). By so doing Borras supports Swain's (1985) recommendation for providing an opportunity for comprehensible output. Borras concludes that her program only helps students in the preparation of actual language use and does not replicate actual interaction. Borras, like many others, projects that as 
technology improves so will the computer's capabilities of oral task feedback and assessment.

Participating in real world language use can also be a goal of software. An example of a simulation that prepares students for real-world interactions in another language is the program $A$ La Rencontre de Philippe. This program is recommended by Rowekamp (personal communication, March 5, 1997) as an exceptional use of simulation where students, either working alone or in small groups, negotiate with the computer to reach an outcome. A branched story of a man looking for a job and an apartment so that he can reconcile with his girl-friend provides the user with different outcomes. Fulfillment of sociolinguistic tasks where students practice both linguistic function and form allow the progression of the story.

\section{$\underline{\text { Student Attitudes }}$}

Student variables like attitudes toward working with a computer are also considered when using technology. If students do not have a positive impression of technological movement and use outside of the classroom, they will not be enthusiastic about technology when face to face with it inside the classroom. There is evidence that most students, when working with computers, are positively motivated. Windeatt (1986) noticed that students liked using computers; these same students would often stay longer than the usual class time so that computer tasks might be finished. Ganszauge et al. (1994) also reported that students in an ESL classroom, who initially had a negative attitude toward computer use before actually using the computer, developed a more positive attitude by the end of the study. They also found that there were very few computer related problems that pertained to students knowing how to use a computer, or becoming confused and disoriented by the tasks. In both 
studies, students displayed behaviors and attitudes that reflected the stimulating environment and relaxed atmosphere the computer can provide.

Other aspects of working with the computer in the classroom fit into a communicative framework. For one, the student is given control if there is a one to one ratio of computers to students. Stevens, however, noticed how students usually group around the computer. He attributes this to a lower anxiety level resulting from students not feeling as if they are being individually observed (1993). Allowing the learner to control the pace of interaction can additionally enable students to not feel pressured when at the computer (Kenning \& Kenning, 1990). Finally, not having to outwardly perform in front of the class or for the teacher is thought to lower the affective filter (Krashen \& Terrell, 1983; Kenning \& Kenning, 1990), thus providing a more comfortable environment in which to learn. Cheung and Harrison's study (1992) of students and adventure games suggests types of activities that keep students engaged at the computer. Cheung and Harrison found that move-based simulations (where the students are confronted with a problem and the computer waits until the students make a decision before progressing with the program, versus action-based simulations where the action is ongoing and the program does not wait for students to take the time for decision making) perk interest and keep students motivated because there is an element of risk involved in the decision making.

The most powerful feature about computers is that if they can make learning a more enjoyable experience, students will have a new source of motivation for learning. Stevens notes that when students start to see the computer as a facilitator of learning and not as a performance medium, they will relax and enjoy the experience of CALL (1993). As can be seen, the computer can be incorporated very nicely into a communicative learning framework. Affectively the computer offers a low anxiety 
atmosphere. Students can engage collectively, cooperatively, and communicatively in tasks in which the focus is on the learner.

\section{Communicative CALL Material}

The creation of ESL CALL materials like any other ESL materials should reflect current theories of language use, language teaching, and language learning (Brown, J. D., 1995; Hubbard 1992). As discussed earlier, theories of language use in the communicative classroom encompass language used in a variety of everyday situations, with every learner having varying needs of language use. Language teaching theory will then structure the content to be learned into manageable chunks based on instructional objectives. This structure specifies the type of syllabus. It is not unusual to find syllabuses organized using a combination of syllabus types (Brown, J. D., 1995). Hubbard (1992) suggests that the considerations used for the organization of any classroom's syllabus and method of teaching can be transferred to the creation of language software.

\section{$\underline{\text { Interactive Activities }}$}

Once the syllabus type has been decided, the next step is to determine various activities that will support the learning objectives. Communicatively these activities will have a high interactivity between students as the exchange of information and the process of negotiation transpires. CALL activities will have the computer acting as the deliverer of language material. Since human interaction usually enhances language learning (Davey, Jones, \& Fox, 1995), activities with a high degree of interactivity are desirable. The programs referred to earlier (i.e. ELEONORE and $A L a$ Rencontre de Philippe) are model programs. Students are given the opportunity to 
initiate and create various outcomes, and still learn sociolinguistic as well as grammatical information. Truett and Gillespie (1984) state that the ability to actively involve the student in his or her own learning is the micro-computer's chief advantage over any other instructional delivery device. Because of this, it is extremely important that educational programs designed to be used with the micro take full advantage of this interactive potential. ( $p$. 43)

Hubbard provides a partial list of design criteria for today's CALL activities (p. 1992, p. 49):

1. Meaningful practice, not mechanical practice

2. Discourse needs to be in larger segments, not one word exchanges; sentences must be meaningful

3. Clues need to be provided that lead students to correct answers

4. Feedback must be facilitative, providing the option of knowing the reasons behind the answers

5. Allow for multiple/alternative answers

6. Anticipate student errors and give helpful feedback

Liou (1993) refers to activity types as those based on remembering, and those based on doing. In a communicative classroom activities based on language use in real situations (i.e. "doing") are optimal. In CALL activities with the use of various media, students can receive contextual clues governing language use, see language in real situations, practice their understanding of the use, and apply language to other situations. Stevens (1993) stresses that in regard to communicating the program does not have to be based on complicated parsing algorithms. He describes a program where students communicated with multiple choice responses, based on a previously 
viewed video. The computer would then react in accordance with the student's response. For example, if a student made a choice that might be interpreted as culturally rude or inappropriate, the computer program would respond by showing a video segment of the character walking away in disgust.

The interactivity of the above program is based on a multiple choice (drill and practice) template. However, the environment is contextual, and the feedback results in real-world consequences the student is indirectly involved in. Davey et al. (1995) stress the importance of not falling back into the meaningless drill and practice activities, especially the one word responses that do not support the communicative focus. Kenning and Kenning (1990) add that the students need to feel as if they are "participants in an exchange" (p. 40). Today's technology now has the tools to use the drill and practice activities of past programs in a more dynamic and contextual environment based on language use.

\section{Quality of Interaction}

Without a human component, interaction and quality of interaction are vital. It is up to the developer and instructional designer to provide the affective motivation that keeps the user moving from screen to screen, and exercise to exercise, and not toward the exit command. Schreck and Schreck (1991) point out that interaction needs to be frequent. Students need to be acknowledged by the computer. Questions to the users are a simple form of engagement (e.g. Are you still there?). Games, branching stories, and role-playing are also forms of maintaining interest and interaction. Besides providing highly interactive activities, the content or topic must have a visible relevance and application for the learner. Davey et al. (1995) support the need for students to have choices about content material to best fit their interests. The inclusion of relevant materials can provide a motivating and stimulating 
atmosphere for students. Lyman-Hagar (1995) explains that an enhanced interest by the students can prime the way for language input to be internalized.

\section{Provision for Culture Learning}

Culture learning can also be adequately accounted for in CALL material. As mentioned previously the real-life applications needed for student interaction are by their nature culturally bound. As such, students need to be given the opportunity to view culture at work; CALL materials must provide the chance for students to try out language before actual use. Pacino and Pacino (1996) relate how interactive media can provide the environment where activities produce cultural experiences and varying levels of intercultural interaction. The program Pacino and Pacino created, an interactive videodisc, presents varying contexts with problems that need solving. Students must decide the most acceptable response; if the response is inappropriate, students are left to reflect upon their decision and consequences of that decision. Pacino and Pacino agree that the realistic encounters can give meaning to abstract cultural concepts. Their program provides the students a comfort zone for culture learning, similar to Kramsch's third area (1993) where students come into contact with and react to culture.

\section{$\underline{\text { Student Consideration }}$}

\section{$\underline{\text { Learning Styles }}$}

Beyond the need for relevance, CALL materials must adequately provide various environments to match learner preferences. The combination of media provides different types of input that can support student learning styles from task to task (Rühlmann, 1995). For example, Liou (1993) reports that pictures are coded both verbally and visually, and as a result are better retained for both visual and textual 
learners. Media must not be misused, however. Flagg (1990) reports on a study that found a student preference for graphics that conveyed information not found in accompanying texts. Media must therefore be used to complement each other and provide further learning information. Used in varying amounts together, media can provide benefits to students by supplying sources of input (Brinton, 1991). Overall, media must be used in a way that supports learning objectives without distracting from the activities taking place.

Stevens (1993) suggests future programming be able to recognize student needs and learning styles by choices students make in the program. The computer could then alter the program according to learner's replies (i.e. make the learning path based on student input). By the storing and analysis of student responses the program is able to adjusts the amount and type of input to the student, the same way a classroom teacher makes a mental note of student learning preferences and then plans future lessons accordingly. Students can then indirectly organize a learning route that makes sense to them (Davey et al., 1995).

\section{Student Control}

Stevens goes on to say how the trend toward providing options for learning gives students a certain amount of control. The provision of various components can ease a student's feelings of being dominated by the computer. Allowing the student some knowledge of and control over the program will maintain a positive attitude toward the computer. For example, through the simple provision of goals and objectives, students are kept informed of the purpose of their work. Borras' program (1993) used the following instructional design considerations: free choice of activities and tasks; record keeping to show completion of learning modules; easy program exit and reentry. The introductory screen on her program orients the users to objectives, 
directions on navigating, and specifics of how to access the modules. Also available is information on grammar, learning how to describe something, and learning vocabulary (allowing users to practice words from the video). Available to the instructor is a screen that allows the monitoring of student time on task, as well as the setting of task levels and video segment difficulty.

$\underline{\text { Issues of User Friendliness }}$

Liou (1993) proposes nine "golden rules" (p. 88) for interactive materials. Several of the rules address user-friendly issues. One of her first rules is to keep a consistency to the program design and layout in order to minimize the visual search time. Referring to interface layout and design, Stevens (1993) also stresses the need for the student not to be distracted from learning by trying to locate icons or program operation instructions, etc. Rühlmann's program (1995) consistently uses the bottom of the screen for navigational tools. She also keeps similar icons together.

Another of Liou's rules states how frequent users of a program should be provided shortcuts to bypass such features as instructions. If students already know the directions, they should be able to bypass them to start right in on an activity. Likewise, with lengthy introductions into a program, it should be made possible for students to turn off the introduction. Another shortcut feature would be the provision of bookmarks that let the student access a specific activity without having to go through multiple screens to get there. Key commands instead of just using a mouse can also be made available for frequent and knowledgeable computer users. Navigational tools will prevent students from getting lost, and good tools will give the seasoned user the immediate access they so desire.

A hyper-environment has the potential of leading students off the learning path. As students use the various programmed options (i.e. Help or on-line 
dictionaries, etc. ) they need to be able to get back to where they started. Chan and Liu (1992) propose using only two levels of hyperlinks. More than two might cause students to get distracted from the task, and then get lost. Besides keeping links at a minimum of two, another tool to keep students from getting lost as well as allowing facilitated navigation is the use of a table of contents. A table of contents will let users know their location, and preferably know it at all times (this is similar to the bookmark feature referred to above). Knowing where one is in a program is akin to knowing what page one is on in a book. Rühlmann (1995) acknowledges the need for learners to be informed at all times about location, as well as what activities have already been completed. "[Reducing] short-term memory loads," another one of Liou's golden rules (1993, p. 88) can be accomplished by simple additions like a table of contents.

Beyond using the table of contents to reduce short-term memory load, having access to help features and directions on an as-needed basis also frees up the student to focus on the task at hand. Knowing these features are available at all times also gives the student peace of mind when operating the program. Since the learner "needs to understand [the] demands made upon him/her without available human help" (Davey et al., 1995, p. 34), help features are greatly needed in CALL programs. Stevens (1993) notes that the use of accompanying manuals should not be required; a good program should be intuitively accessed. If it is not, Help and directions should be available, although hidden. Too much dependency on Help is not beneficial. Learners exploit it and then become passive participants of the program (Davey et al., 1995). In reference to the amounts of Help required, Davey et al. (1995) also point out that if there is too much information the program can appear to be patronizing. Yet if there is not enough, confusion and frustration may follow. Animated demonstrations may take away the anxiety of having to read the directions, especially if the directions are not in the student's native language. 


\section{$\underline{\text { Importance of Feedback }}$}

Feedback is an essential component of CALL material. Feedback in the communicative classroom allows students to gauge their developing abilities and success through interactions. This sort of feedback also needs to be provided in the software. Schreck and Schreck (1991) mention that quality feedback needs to be appropriate for the task, directly related to the learner's input, easy to interpret, and positive. This would mean that feedback that gives the same answer over and over is not sufficient. Students will become used to the feedback, get bored, or even lose interest in the activities. The same applies to errors. Programs that just give a "wrong, try again" response are nothing better than the behaviorist drills of early CALL materials. Communicative classroom responses to errors must "provide opportunities for learners to recognize the communicative effectiveness of their target language productions" (Crookes \& Chaudron, 1991, p. 61).

A computer's response to errors will be within the parameters of what it is programmed for. If it is programmed to say "wrong, try again buddy" after every incorrect response by the student, then it will do just that. However, programmers have other alternatives to "wrong, try again" models. Taken from Higgins and Johns (1984) and Schreck and Schreck (1991) the following options are available as forms of feedback: move the cursor to the next line; provide animation; show a printed message; let the learner know a wrong answer was given and then continue with the program; show the correct answer and continue on; let the student try the problem again, if they so desire; don't tell the student anything, and just have them try it again; tell the user the response was wrong, and store the question so that it comes up again later; ignore the mistake and move on. 
Although the options for feedback given above don't show it, the main point to remember is that feedback needs to be constructive, and useful within the contexts of a communicative environment. It should never be the same feedback repeated again and again. The earlier programs discussed in this chapter all used different kinds of feedback. The nature of the program will most likely determine the type of feedback and error response. If the program is really geared toward communication, error correction will show communicative breakdowns because of misuse of linguistic forms. Also, more than one answer should be accepted because of the unpredictable flow of communication (open-ended questions are more common in the communicative classroom). If the program is behaviorally based, only one answer will be accepted (because the questions asked are more close-ended). Liou (1993) says that above all, feedback needs to be informative. Rühlmann (1995) stresses the importance of students not being judged until the tasks they are working on are completed. In a non-testing situation this is very important, for students may change their minds. Yet even in classroom testing situations students are able to change their responses. Liou (1993) proposes another golden rule: allowing students to easily reverse their actions or decisions. 


\section{CHAPTER III}

\section{METHOD}

This study assessed current instructional design features of ESL CD-ROM software relevant to today's communicative teaching approach when a human instructor is not present. Eight software packages were selected and then evaluated using a tool created specifically for the study. The evaluation instrument was composed of various viewpoints within the ESL, General Language Learning (GLL), Educational, and CALL fields, allowing a more encompassing and comprehensive evaluation than if only one field's evaluative criteria were represented. The process of the evaluation proceeded in a three-step manner. This chapter presents the selection criteria used for the software, the creation of the evaluation instrument, and finally the method used for the instrument's application. Limitations of the study are also addressed. Results in Chapter IV are shown in a matrix which serves as a quick reference to the results of the study; the matrix should not be regarded as a stand-alone tool as it does not address theoretical underpinnings of the criteria. The final treatment of the findings is presented in Chapter V, appearing in essay format. 
General Design of the Study

\section{Identification of the Software}

A lot of ESL software is available, both commercially and as share-ware. After researching I found that ESL software in CD-ROM format is not as widespread as other CD-ROM material not developed specifically for ESL. ESL may not be a lucrative enough marketplace to warrant large numbers of CD-ROM ESL products. The monetary cost of a quality CD-ROM program, because of the labor-intensive work required to successfully integrate the various media found in multimedia software, is much higher than that of a text-only behaviorist drill and practice disk program (which because of its size does not need the storage available on the $\mathrm{CD}$ ).

While other evaluative studies may include software not specifically developed for ESL (Carrick, 1988), I chose to include only ESL material. I wanted specifically to see if the ESL material exhibited a sensitivity to the needs of second language learners when the instructor is not present. These needs, discussed in Chapter II are interactivity beyond drill and kill programs, culture learning and pragmatic language use, and varied sources of input. I also opted to confine the study to commercially available material. While many university-based individuals and small groups create their own material (for example the ELEONORE program by Renié and Chanier, 1995, referred to in Chapter Two; Borras' program Practicing Spoken French, 1993), unless marketed and thus made available to a widespread audience, these model programs remain outside of mainstream CALL. Commercial products are representative of the perceived needs of large numbers of instructors and students, not of smaller institute-specific programs. The latter, as seen in the descriptions in Chapter II, are more grounded in theory base. Commercial products, by their nature of 
being widely available, require a more established theory base so that state of the art CALL material can begin to separate itself from the past behaviorist images.

The goal of this study is to evaluate communicative based software (i.e. software more grounded in the communicative approach to teaching than the past drill and practice software) in order to assess the current conditions when a human instructional component is not present. Not all the CD-ROM material developed for ESL automatically meets the criterion of being communicatively based, as outlined in Chapter II. CD-ROM material can fall under three broad paradigms: the data-base, the tutorial, and the paradigm. Looking at marketing literature found mainly on the World Wide Web, I assessed the programs to identify those that might serve as the representative sample of the study. I was looking more for tutorial-based formats. I disregarded those programs advertised as "interactive phrase books," "picture dictionaries," "narrated stories," or "song-based." Programs of this nature did not seem to be based on a communicative approach to language instruction and learning. Instead, I searched for material and found some (separate from the song and story programs) advertised with copy like "Learn to read, speak, and understand English"; "contextualized exercises"; "gain proficiency by providing interaction with native speakers in real-world situations"; "build a strong base in both spoken and written English."

I could not find any software that claimed a communicative based theory of language learning and teaching. Perhaps the term "communicative" is being avoided because of what could be to some a misrepresentation and misapplication of the term. Perhaps there is no communicative-based software. Since computers do not and can not actually communicate in a human-like, authentic manner, they can not truly be communicative when compared to an ESL setting that claims the same feature. 
Instead, terms like "total immersion," "proficiency oriented," and "natural approach" describe the software.

To broaden my search for more communicatively based commercial ESL CDROM material I consulted the 1995 CALICO Resource Guide for Computing and Language Learning; ESL and general education material catalogues; various publications for software review information; local computer stores; and colleagues. The ORTESOL Spring 1996 conference provided a lead, as did information obtained from the Oregon Multimedia Education Conference in Fall, 1996. After a general group of software was identified, a number of factors which could have affected the software's selection were "neutralized" so as not to further limit the small number of software packages available. These factors were cost, content, and intended user age.

The price of software sought for this study ranges from $\$ 100.00$ to $\$ 1,000.00$ per package (an evaluation of database software would have been less expensive; prices are from $\$ 30.00-\$ 50.00$ per package). I created a strategy to make the price of the software "affordable" for a student's budget. Software companies were approached and offered participation in the study. Most companies expressed a desire to be included; in return for their participation each will receive a condensed version of my findings (in short, I did free market research for them). Two companies who were unwilling or unable to participate in the study offered a $30-45$ day viewing period before billing me for the products. Overall, seven companies are represented in this study (Appendix A).

Content refers to the basic underlying theme of the material. For example, a CD-ROM English for Specific Purposes (ESP) course of business English will focus on business applications of English language use. However, it did not matter if the material was about business English, or if the material was a basic English learning program; both programs were equally considered. Content per se becomes secondary 
to its presentation through the various media, and to the way software designers provide the various design features needed for a learning/instructional situation where a human is not present. The same applies to intended user age. Again, it is the learning environment and the instructional design features of the ESL CD-ROM material that are relevant. Whether the material is for six-year-olds, or sixty-year-olds, certain instructional design and technical features (as identified in a number of sources to be explained in detail in General Design of the Study, this chapter) should be made present in the program. The user's age was only considered as it applied to the relevance of instructional design.

I had initially wanted to include "hardware requirements" as a factor to be "neutralized" for software selection. I did in the end decide to limit software based on hardware considerations to those being state of the art, with easy installation for Windows or Macintosh, and not DOS-based. Blum, in 1995, remarks that any competitive commercial material should not be DOS-based. In 1997, where operating systems and upgrades can easily occur within six-months, any use of or "[design] for yesterday's technology" (Blum, 1995, p. 28) can be hazardous to the success of the product. While many schools may have older DOS-based operating systems, technically I wanted this study to include software current with today's commercial market and competitive nature. As a result of this one limitation, I sent one software package back to its developer.

A total of eight CD-ROM ESL multimedia programs was examined (Table I). 
TABLE I

Software Evaluated in the Study

Learn to Speak English

Ellis Senior Mastery

Rosetta Stone English, Levels I \& II

Dynamic English, Levels I, II, \& III
Let's Go, Levels I \& II

TriplePlayPlus!

Focus on Grammar

Interactive Business English

\section{Identification of Evaluation Criteria}

The evaluation of computer-based materials is treated much like other instructional tools (Schreck \& Schreck, 1991): materials are identified, an evaluation tool based on the anticipated outcome and use of the results is applied, and, in a systematic fashion, the review process takes place. Schreck and Schreck point out that there does exist a difference between a CALL material evaluation and a non-CALL material evaluation; the need for a closer examination of the learner-computer interaction taking place without a human instructor present distinguishes the two evaluations from one another.

As the purpose of this study is mainly to determine how the instructional design of material delivered through the medium of the computer compensates for the lack of a visible human instructional component (which includes Schreck and Schreck's concern for examining the interaction between learner and computer), the criteria and tool for the evaluation need to be more focused than the general categories found on many software review sheets (Meskill \& Swan, 1993). Many of these review sheets (see Eisele \& Eisele, 1990; Truett \& Gillespie, 1984), besides containing categories of general information that include operating system requirements, type of 
program (i.e. word-processing, class scheduling, etc.), and reports generated, include criteria addressing whether content objectives are met through the program; basically, how well the program fits into the course needs in terms of content, scope and sequence are the main points addressed.

While judging whether a program based on its content should even be initially considered for inclusion into the curriculum makes perfect sense, instructional design issues are relegated to the end of the review sheets. The design features like ease of use or user orientation are used to further separate the programs once instructors have made general selections. As this study addresses instructional design issues directly, it was necessary to create a tool based on a number of sources in order to make instructional design criteria the focus. These sources, as mentioned earlier, represent the ESL, Education, GLL, and CALL fields. As a result, a more comprehensive tool based on the needs of the study was created.

Schreck and Schreck (1991) explain how the learner-centered nature of CALL materials necessitates a more thorough examination of the interaction taking place between the learner and the material. Without the human instructor guiding the interaction, clarifying content as needed, gauging comprehension, and providing feedback, the student using CALL materials is left to the pre-determined consequences of the program's instructional design. In the evaluation of courseware, Schreck and Schreck stress that beyond the standard evaluation criteria that can be applied to both CALL and non-CALL materials, the provisions made for the lack of a human component must be closely examined. The criteria Schreck and Schreck propose encompass the quality of the general instructional design (this includes the handling of instructions, the number and type of questions asked, the answer responses, and the feedback), and how well interaction can take place between the user and the 
courseware features (these include both mechanical features and aesthetic features of the program).

Eisele and Eisele (1990) and Truett and Gillespie (1984) represent the general education field. Both teams examine the evaluation of technology used in general education with both supporting criteria parallel to those supported by Schreck and Schreck. Eisele and Eisele, for example, offer a number of checklists with instructional and technical design criteria. Truett and Gillespie consider the user's interaction to represent one of their guiding criteria; issues like the program's userfriendliness and handling of feedback are also addressed.

Beyond the three sources of Eisele and Eisele, Schreck and Schreck, and Truett and Gillespie, Liou (1993) proposes a number of underlying principles to consider when designing CALL interactive systems (some of these principles were discussed in Chapter II). Liou's principles were used to create a guideline for criteria addressing user-friendliness and interaction quality. User-friendliness of software programs is also referenced by Flagg (1990) in her work based on the formative evaluation of educational technologies. Flagg likened a good computer program to a good butler, in the sense that both should be responsive, accessible, flexible, and able to remember individual particularities. Extending Flagg's butleresque qualities of a good program to areas to consider when one examines the worth of a program, the same can be applied to interactive qualities (responsive and flexible), user-friendliness (accessible), and record keeping (good memory).

Two sources drawn from an ESL background offer additional criteria on the non-human instructional component. Brinton's contribution (1991) to the criteria is based on media use. Multimedia, as the word implies, is the combination of various media. In a computer setting, the use of multimedia assumes the same role and associated usefulness of a non-computer application; that is, it is used to support and 
reinforce teaching objectives. Without a human instructor present, it becomes even more critical for instructional designers to have carefully thought out the inclusion of media and its delivery through the program. Although not an ESL source, Brad Hanson, Director of Distance Learning at Portland State University's School of Extended Studies, stresses that there is a need for multimedia for the many different learning styles students have, but that the media must also be appropriate for the content being delivered (1996).

Wasted use of media may lead to confusion for the user and distract from the learning objectives. It has been pointed out that not only do the media need to be purposeful, but that the learning exercises should draw from the enriched context media can provide (Coleman, 1991). Skierso (1991), another ESL source, offers tips on the evaluation of textbooks that include evaluation criteria to judge the effectiveness of media in the form of text photographs and drawings. I used Skierso's criteria not only in reference to media, but also for issues of user-friendliness.

The focus of this evaluation is not on content. Instead, the presentation of content and the instructional design features through the medium of computer programs are being examined. To be competitive, however, the content of today's software needs to reflect the relevant issues surfacing in many ESL classrooms. The learning of language and its many sociolinguistic variants found in a number of situations is becoming more and more viable. Therefore, a brief survey of the communicative content and the portrayal of culture provides a look at how the advantages of technology are being used in today's CALL materials, if at all.

In contrast to the computer's actual communicative real-world speech abilities, the portrayal of culture and its inclusion through contextual communicative input has numerous opportunities and possibilities in multimedia CALL. Texts, dialogues, artwork, and video can provide culture-rich and communicative input that students can 
learn from and use away from the computer and ESL classroom. As much as effective instructional design needs to account for a teacher's instructional absence, the specific cultural and contextual language input and supporting activities need to be accurate, realistic, and free from blatant hurtful biases and cultural slants. Opportunities need to be provided for learners to become cultural learners, aware not only of others as people of culture, but of themselves as culture bearers.

Cohen (1996) refers to the specific ability of performing speech acts (which is not included in the scope of this study) when he asserts that "the existence of accurate pragmatic information may not be enough if that pragmatic information is subjected to 'defective presentation' by teachers “ (p. 264). His claim can also be applied to the presentation of sociolinguistic and cultural information through CALL. Not only does pragmatic information need to be accurate, but exercises need to give students the opportunity to use the information, seeing how language use and linguistic formulas vary with every situation. Renié and Chanier's (1995) program discussed in Chapter II shows how the computer can act as a guide whose help enables students to learn the subtleties of communicative language use.

In the creation of materials, Nelson (1995) remarks on ESL/EFL writers' need of cultural awareness; it is through materials that culture is portrayed, whether knowingly or not. Nelson's idea transfers easily to CALL. Although I did not intend to minutely examine all the culturally bound elements in the program, from layout and interface design to the structure of activities, criteria regarding contextual communicative input with its visible relevance and representation of culture are included.

After the various sources have been combined, the criteria for the evaluation now fall under the following categories: feedback and error treatment; relevance of input by the various media and aesthetic surface features; type of interaction (both 
quality and quantity); software's user friendliness and learning environment; record keeping capabilities; sociolinguistic input and practice; communicative language skills that include communicative strategies. The letters I sent to the various companies made reference to these categories as areas of research. Sue Otto, Director of Instructional Design at CALI, regarded the categories as reasonable for an evaluation of this type (personal communication, January, 1997).

\section{Creation of the Evaluation Instrument}

As I established the general criteria from a combination of sources, I also created the evaluation tool by a similar process. Using Skierso's method of applying numerous sources into one comprehensive checklist (1991), the specific evaluation tool for the study was composed. As mentioned earlier, this tool draws the most relevant criteria from various sources together to allow better access to the non-human instructional components for the evaluation (this is in reference to the first five categories listed above) plus the inclusion of culture and communicative language use (see Appendix B for the sources and checklist). The criteria were rated using likert scaling. Many of the checklists in the research literature used likert scaling in their procedures. Again drawing from Skierso, the rating scale was exactly as suggested (1991, p. 441): 4 = Excellent; $3=$ Good; 2 = Adequate; $1=$ Weak; $0=$ Totally Lacking. The checklist is divided into the seven major areas for the evaluation. Extra space is left under each question for the collection of notes to aid in answering a number of summary questions as proposed by Eisele and Eisele (1990). These questions, found on the last page of the evaluation tool, are discussed in both Chapters IV (the results) and V (the discussion).

A matrix of the findings (Table II, Chapter IV) presents the results in an easily accessed manner. At a glance, the viewer can look across the columns to see how the 
software in general rates for each criterion. While the matrix contains valuable information pertinent to the study, the matrix must be viewed with knowledge of the theory backing the criteria. The matrix is not the final treatment of the findings; it is a quick reference tool. Instead, the notes collected, together with each criterion's rating, are discussed as a whole in Chapter $\mathrm{V}$ in conjunction with the research questions.

\section{Review Process}

According to Nunan (1992) the design procedures used in a survey study follow the identification of the sample (in this case it is the software) and the specification of the evaluation instrument; the design procedure of any gathering of data then proceeds in a methodical fashion. The evaluation of software, however, proceeds somewhat differently than the evaluation of materials such as textbooks. Due to the nature of the learner-directed interaction without the close supervision of an instructor, software material requires "a much more thorough evaluation than is ordinarily required for the traditional instructional programs and materials" (Schreck \& Schreck, 1991, p. 478). Textbooks do not need to be evaluated for issues of user friendliness or quality of feedback. Perhaps the content of texts will intimidate students, but the technical operation of a left-edged bound book is almost universally taken for granted. Software, however, is different. The student using the program must, right from the start, experience a learning environment conducive to effective language instruction. Without this facilitative environment that hinges on the program's instructional design qualities combined with engaging and relevant content, the experience may sour the medium's perceived instructional value.

A general three-step examination process is proposed for the evaluation of the software once both the suitable goals of the evaluation, and the software, are identified 
(Schreck \& Schreck, 1991; Truett \& Gillespie, 1984). These three steps make concessions for three different viewpoints: the student's, the instructor's, and the instructional designer's (teachers assume the role of the instructional designer when evaluating instructional design criteria). Student viewpoints focus on mechanical (i.e. user friendly) and aesthetic qualities; the instructor looks for how well the program fits the classroom's needs (i.e. scope and sequence); the instructional designer looks to the quality and organization of the content, as well as the interactivity provided. The evaluation then proceeds three times through the material; each pass through has the evaluator assuming a different perspective from which the evaluation takes place. If students are used during the evaluation, the instructor then assumes only one extra role, that of the instructional designer.

Schreck and Schreck (1991) propose their three phase examination where the evaluator plays the role of the student, but they do not say anything about using actual students for the evaluation. Truett and Gillespie (1984) do note that student input is extremely relevant, but they also realize that often only one person is "forced" (p. 41) to evaluate the software. The number of different runs through the software is meant to compensate for the lack of student input. Because of time constraints on this study, I opted for Schreck and Schreck's individual evaluation routine where I based each run through the program on criteria specific to the viewpoint of the role I was taking.

This study is for the discovery and examination of instructional design issues based on content delivery through the medium of a computer program. As a result the criteria chosen and the structure of the review process are based on reaching this goal. For this study, the review process focuses mainly on two of the three steps discussed earlier: the students' viewpoint with issues of user-friendliness and learning environment, and the instructional designers' viewpoint built upon interactional qualities, relevancy of media, and record keeping. To account for issues of culture and 
communicative language input I did take an instructor's viewpoint but I kept instructional design and student issues as the main areas of research.

The software was still evaluated in a three phase process, modified for the study. I spent a period of approximately five to seven hours on the evaluation of each package. Those packages containing a number of levels required between ten to fifteen hours for their evaluation so that each level could be independently evaluated; any divergence in the rating of criteria for each level is noted in the matrix and in the summary questions answered in Chapter IV.

The first phase was based on student issues. To compensate for not using students, I accomplished this section of the evaluation by taking an initial "naive user" stance. Although not all ESL students will be computer-naive, the role of the naive user allows the evaluator the opportunity to put previous computer knowledge aside and play a role as if one was computer-naive. A naive user, in my interpretation, has little if no experience working with computers and as such does not hold any experientially based expectations of computer programs. To play this role, after getting into the program and to the main menu screen (after installation almost all programs quickly delivered the student to the main menu), I sat and waited for computer guidance, or I either cautiously or zealously explored on my own (I tried to play a number of different personalities; not all personality types are represented). I kept a log of my progress (see Appendix $\mathrm{C}$ for sample pages), noting my frustrations, my capabilities, my likes and dislikes, any big surprises, and my general feelings toward the program. By this method I maintained as best I could an open-mindedness toward the first part of the evaluation by looking at the program through the eyes of an essentially inexperienced user.

While my knowledge of computer terminology shows up in the log (I do call the "mouse" a "mouse"; I do not even attempt to come up with a descriptor that an 
ESL student might use if less knowledgeable of the technolect), my assumed naïveté allowed an assessment not fully formulated upon my prior experience, expectations, or knowledge. Anyway, I am just as novice-oriented in CALL as the naive user; although my expectations are theoretically based, my real-world knowledge, similar to that of CALL evaluators used for another study assessing software, is "untainted by prior experiences" (Meskill \& Swan, 1993, p. 105). Throughout the entire evaluation I relied only on the software for information. If written material accompanied the program, I referred to it only after the evaluation as a student was finished. By so doing more of a genuine naïveté of not knowing in advance what the program expected me to do was kept. After getting a general understanding of the program with regard to user-friendly issues, and after answering the criteria, I changed my assessment role to that of the instructional designer.

As an instructional designer I was concerned with issues of feedback and error treatment, the relevance of the media and aesthetic surface features, the interaction created by the programs, and the record keeping capabilities. To assess these components I again went through the software with the specific criteria in mind. I repeated the different activities, scrutinized the use of the media, and noted the different types of activities and interactivity. Working on a section at a time I proceeded through the entire program until I felt justified with my ratings. The notes I took reinforced the ratings and allowed me to recognize whether I was consistent with what I was looking for.

To address the last components of the survey where issues of culture and communicative language input are examined, I once again went through the program. From the other passes through I had already experienced the software's portrayal of culture and communicative input. This third phase, where I took on more of a teacher role, allowed me to actively examine the program to rate the criteria. 
I initially expected to provide an objective assessment of the program by doing a run-through of it before conducting the subjective assessment. The objective review would provide a brief description of the program containing information such as the number of disks, the level/age of intended users, and the general features of the program. Meskill and Swan (1993) suggest an objective description of programs before administering the subjective interpretive criteria. Hutchinson and Waters (1987) also agree that an evaluation needs to contain both objective and subjective analyses; without the objective review, they caution, the subjective criteria may obscure the objective features of the program. According to Meskill and Swan, the objective information can "serve as the base into which further subjective commentary [can] hook" (1993, p. 105). I did do an objective assessment, but only after the "student" evaluation was complete. If I had done the review earlier, I could have been biased by knowing in advance what to expect from the program.

The findings of the study, as addressed earlier, are presented in Chapter IV in a matrix for quick reference, with a more thorough subjective evaluation for each software available.

\section{Limitations of the Study}

This study is limited to only one person's evaluation; this is the largest limitation. Without using student test groups or professional consultants to judge the validity of the findings, the study is not as strong as it could be if students and consultants were used. Due to the essentially subjective nature of the ratings, there can be no assurance that someone else looking at the same software with the same tool will get the same results. If I had devised an exact coding method for each of the criteria, perhaps consistency could be maintained for greater inter-rater reliability. 
It does seem that the study might accurately reflect current conditions for the evaluation and assessment of software (I am making reference to how instructors might come upon software and then conduct a single person evaluation). Schreck and Schreck (1991) obviously appear to think this behavior is typical, for they make no reference to even including students in their evaluation, and they only briefly mention consulting others (e.g. other instructors). The process of the study was meant to cover all important angles, and the measures taken (i.e. the "naive-user") provide a more thoughtful approach to the evaluation than if the measures were not taken.

The selection of software also holds some flaws. Perhaps more applicable software were available but overlooked. Perhaps during the time of this study newer software were produced. Therefore the internal reliability for the study is weak.

Because of the nature of the software industry where upgrades are a matter of survival, the changes done may render the findings of this study obsolete. Also, the understanding and study of how people learn languages is always open to interpretation, and the theory base I worked from may not seem plausible to future evaluators. 


\section{CHAPTER IV}

\section{RESULTS OF THE STUDY}

The following chapter provides the results of the study. Table II provides all of the ratings obtained for each software piece. Each of the eight software reviewed also has both an objective and a subjective description. The objective information (essentially describing the level of the program, the basic organization of content, and the program's features) is located in the beginning of the review under "Program Description." The subjective evaluation remarks based on the summary questions of the evaluation tool (addressing the program's strengths and weaknesses and how the program takes advantage of the computer's instructional strengths) follows under "Program Strengths and Weaknesses" and "Program Instructional Strengths." The section titled "Program Recommendations" completes each of the reviews.

It must be cautioned that the reading of only this chapter will not provide the theory base guiding the study and upon which the evaluation is made. Expectations of the programs and the associated evaluations are in conjunction with the theory presented in Chapter II supporting today's communicative-based ESL CD-ROM material; the findings do not fully consider the developer's intentions for the individual programs. The use of this section as strictly a buyer's guide is discouraged. 
TABLE II

Results of the Evaluation

\section{SOFTWARE}
A $B$
C D
E
F
G $\quad \mathrm{H}$

CRITERIA

\begin{tabular}{|c|c|c|c|c|c|c|c|c|c|}
\hline \multicolumn{10}{|c|}{ A. USER FRIENDLINESS } \\
\hline la & Clear instructions & 0 & 2 & 3 & 2 & 2 & 1 & 3 & 1 \\
\hline $\mathrm{lb}$ & Accessible instructions & 0 & 4 & 4 & 2 & 2 & 2 & 3 & 3 \\
\hline $2 a$ & HELP at all times & 3 & 4 & 4 & 2 & 4 & 2 & 3 & 3 \\
\hline $3 a$ & $\begin{array}{l}\text { Learner in control of } \\
\text { pace, manner, direction }\end{array}$ & 4 & 3 & 3 & 2 & 2 & 2 & 2 & 3 \\
\hline $3 b$ & Input can be changed & 1 & 1 & 3 & 0 & 1 & 1 & 0 & l \\
\hline $3 c$ & Choice of graphics etc. & 3 & 4 & 2 & 1 & 2 & 1 & 2 & 0 \\
\hline $3 \mathrm{~d}$ & Access to reports & 2 & 0 & 3 & 3 & 2 & 2 & 2 & 0 \\
\hline $3 e$ & Know location & 4 & 1 & 4 & 1 & 1 & 0 & 1 & 2 \\
\hline $4 a$ & Consistent interactivity & 4 & 3 & 4 & 3 & 2 & 2 & 3 & 3 \\
\hline $4 \mathrm{~b}$ & Clear learning objectives & i & 2 & 3 & 3 & 1 & 2 & 3 & 2 \\
\hline $5 a$ & Easy navigation & 2 & 3 & 4 & 2 & 2 & 2 & 2 & 2 \\
\hline $5 b$ & Shortcut features & 1 & 3 & 4 & 1 & 1 & 0 & $\overline{1}$ & 2 \\
\hline 6 & $\begin{array}{l}\text { Free of cultural \& sexual } \\
\text { bias }\end{array}$ & 2 & 3 & 1 & 3 & $1,1,2$ & 2 & 2 & 2 \\
\hline \multicolumn{10}{|c|}{ B. FEEDBACK \& ERRORS } \\
\hline 1 & Feedback varied & 2 & 3 & 2 & 2 & 3 & 3 & 4 & 3 \\
\hline 2 & $\begin{array}{l}\text { Feedback constructive } \\
\text { for correct answers }\end{array}$ & 1 & 3 & 1 & 2 & 2 & 2 & 4 & 1 \\
\hline 3 & $\begin{array}{l}\text { Feedback constructive } \\
\text { for incorrect answers }\end{array}$ & 1 & 2 & 1 & 2 & $2,2,1$ & 2 & 3 & 3 \\
\hline 4 & $\begin{array}{l}\text { Every opportunity for } \\
\text { correct response }\end{array}$ & 3 & 3 & 3 & 2 & 2 & 2 & 3 & 3 \\
\hline \multicolumn{10}{|c|}{ CI. RELEVANCE OF MEDIA } \\
\hline 1 & $\begin{array}{l}\text { Overall media aids in } \\
\text { presentation of material }\end{array}$ & 3 & 3 & 3 & 3 & 2 & 2 & 4 & 3 \\
\hline a & Photo & 4 & 3 & 1 & 1 & 2 & 1 & 4 & 3 \\
\hline $\mathrm{b}$ & Video & 0,2 & 3 & 0 & 4 & 0 & 0 & 0 & 0 \\
\hline c & Audio & 4 & 3 & 3 & 4 & 3 & 3 & 4 & 3 \\
\hline d & Text & 4 & 4 & 3 & 3 & 1 & 1 & 4 & 4 \\
\hline 2 & $\begin{array}{l}\text { Media is useful as } \\
\text { feedback }\end{array}$ & 1 & 2 & 2 & 1 & 2 & 2 & 4 & 3 \\
\hline a & Photo & 2 & 2 & 0 & 1 & 1 & 1 & 3 & 3 \\
\hline $\mathrm{b}$ & Video & 0 & 3 & 0 & 0 & 0 & 0 & 0 & 0 \\
\hline & Audio & 1 & 3 & 0 & 0 & 3 & 3 & 4 & 3 \\
\hline & Text & 0 & 2 & 2 & 2 & 2 & 2 & 4 & 3 \\
\hline \multicolumn{10}{|c|}{ CII. SURFACE FEATURES } \\
\hline 1 & $\begin{array}{l}\text { Understandable \& helpful } \\
\text { JTERACTION }\end{array}$ & 2 & 2 & 2 & 4 & 2 & 2 & 4 & 3 \\
\hline 1 & $\begin{array}{l}\text { Levels of difficulty } \\
\text { available }\end{array}$ & 4 & 3 & 0 & 3 & 3 & 2 & 2 & 2 \\
\hline 2 & $\begin{array}{l}\text { Reasonable responses } \\
\text { accepted }\end{array}$ & 0 & 0 & 0 & 1 & $1,2,1$ & 1 & 0,1 & 0 \\
\hline 3 & $\begin{array}{l}\text { Questions \& prompts } \\
\text { frequent }\end{array}$ & 2 & 1 & 0 & 1 & 3 & 3 & 4 & 2 \\
\hline
\end{tabular}




\begin{tabular}{|c|c|c|c|c|c|c|c|c|c|}
\hline 4 & $\begin{array}{l}\text { Different questioning } \\
\text { formats }\end{array}$ & 1 & 3 & 3 & 3 & 2 & 2 & 4 & 2 \\
\hline 5 & $\begin{array}{l}\text { Questions relevant to } \\
\text { content }\end{array}$ & 3 & 3 & 3 & 4 & 3 & 3 & 4 & 3 \\
\hline 6 & $\begin{array}{l}\text { Students share in } \\
\text { initiation of events }\end{array}$ & 2 & 2 & 1 & 3 & 2 & 1 & 3 & 2 \\
\hline \multicolumn{10}{|c|}{ E. RECORD KEEPING } \\
\hline 1 & Movement tracking & 0 & 0 & 4 & 3 & 2 & 2 & 2 & 1 \\
\hline 2 & Records available to others & 1 & 0 & 2 & 4 & 3 & 2 & 1 & 0 \\
\hline $3 \mathrm{a}$ & Test results & 2 & 0 & 4 & 0 & 0 & 0 & 0 & 0 \\
\hline $3 \mathrm{~b}$ & Complete records & 0 & 0 & 4 & 3 & 0 & 0 & 1 & 0 \\
\hline $3 c$ & Item performance & 0 & 0 & 4 & 0 & 0 & 0 & 0 & 0 \\
\hline 4 & Test at beginning \& end & 1 & 0 & 2 & 2 & 0 & 0 & 0 & 0 \\
\hline \multicolumn{9}{|c|}{ F. COMMUNICATIVE LANG. } & \\
\hline 1 & Authentic \& meaningful & 1 & 2 & 1 & 2 & 1 & 1 & 3 & \\
\hline 2 & Use of different contexts & 1,2 & 3 & 1 & 3 & $0,1,0$ & 0 & 2,1 & \\
\hline 3 & $\begin{array}{l}\text { Background information } \\
\text { to understand context }\end{array}$ & 0 & 3 & 1 & 2 & 0 & 0 & 2 & 1 \\
\hline \multicolumn{8}{|c|}{ G. CULTURE } & 4 & 2 \\
\hline 1 & $\begin{array}{l}\text { Examination of cultural } \\
\text { behavior }\end{array}$ & 0 & 0 & 0 & 1 & 0 & 0 & 0 & 0 \\
\hline 2 & $\begin{array}{l}\text { Opportunities for culture } \\
\text { hypotheses }\end{array}$ & 1 & 1 & 0 & 1 & 0 & 0 & 0 & 0 \\
\hline 3 & Free of bias, stereotypes & 2 & 3 & 1 & 3 & $1,1,2$ & 2 & 2 & 1 \\
\hline 4 & Culture integrated in content & 0 & 2 & 1 & 2 & 0 & 0 & 0 & \\
\hline 5 & $\begin{array}{l}\text { Culture treated as } \\
\text { supplemental }\end{array}$ & 4 & 3 & 4 & 2 & 0 & 0 & 0 & 0 \\
\hline
\end{tabular}

Note. Refer to Appendix B for full description of the criteria. Each of the Level scores are shown when results are not similar.

Key: Software Identification: $A=$ The Rosetta Stone Level I, II; B = Learn to Speak English; $\mathrm{C}=$ Focus on Grammar; $\mathrm{D}=$ ELLIS Senior Mastery; $\mathrm{E}=$ Dynamic English Level I, II, III; F = Interactive Business English; G = Let's Go I, II; H = TriplePlayPlus!

Scoring: $0=$ totally lacking; $1=$ weak; $2=$ adequate; $3=$ good; $4=$ excellent 
The Evaluations

\section{Interactive Business English \\ (DynEd International)}

\section{Program Description}

Six discs comprise the software package Interactive Business English.

Intended for users who are at a Pre-intermediate (discs 1, 2, 3), Lower Intermediate (discs 4, 5), or Intermediate (disc 6) level of English learning, the program focuses on language learning through the practice of listening. Media used in the program are text, graphics, and audio. Each disc introduces students to specific business themes and concepts like job history, company description, and product comparison. Using a variety of activities centered around true/false, multiple choice, and fill in the blank questioning formats, students take information from the presentation unit and apply the vocabulary and business concepts to activities found in each of the remaining five units. In the presentation unit students are given periodic sets of comprehension questions based on the material presented both aurally and visually (graphically). "Question Practice" has students making questions by clicking on words and phrases; "Dictations" give students practice with listening to a sentence, locating the missing words of the sentence (in text format), and then clicking on the appropriate choice to fill in the blank; "Quantitative English" shows mathematics and scientific language use through aural and visual presentation. Students then answer comprehension questions in multiple choice format. The "Verb Grid" focuses on verb structures. Students then identify the verb-type by clicking on a choice of three answers. 
"Grammar Fill-ins" repeat grammar and vocabulary items of the disc; students then answer fill in the blank questions.

One of the features of the program allows students to at any time record their own voice and then play it back immediately. Translations are available; students can repeat information at any time; even though the program is timed, students can pause the screen for indefinite amounts of time; text can be displayed or not. A "Shuffler" feature raises and lowers the level of difficulty and the amount of information as students respond correctly or incorrectly. Student records are available; these show time spent in each lesson and the most recent score achieved.

The program is based on a text book, but the program does not depend on the text for instruction. A teacher's manual accompanies the material. The teacher's manual includes suggestions for classroom activities based on the $\mathrm{CD}$ material. A study guide is also included in the package. In it one finds instructions on how to do a lesson, information about all the lesson types, and all the directions. The program can be used as self-study; it is also suggested to act as a stand alone basic Business English course.

\section{Program Strengths and Weaknesses}

Interactive Business English has a particular strength in that it allows speech practice. Students can use the record/playback feature and compare their voice to that available on the program. The quality audio provides students with clear samples, albeit scripted samples, of language use. Unfortunately the speech practice is not an integrated component of the lesson; students must feel compelled, without any program prompting, to use the feature by themselves. While this is a good feature to have, inexperienced users may overlook it, or just not quite know how to use it. Another strong feature is the program's attempt to incorporate various levels of 
learning through the Shuffler. The shuffler responds to students' responses by giving more information at a slightly higher level when students answer correctly, or less demanding questions and information when students answer incorrectly. The practice booklet mentions various levels the student should strive for; these levels are used to reflect the proficiency level of the student in the program. A user-friendly strength of Interactive Business English is the frequent use of prompts and questions. If a student lingers for an expanse of time the program asks "Are you there?" and "Do you want to continue?" The program in this manner responds to the student in a humanistic manner by showing some consideration for the user.

One of the biggest weaknesses of the program is that without the explanations in the study guide, inexperienced students do not know what the program features are, nor are they given adequate on-screen access to sufficient and clear instructions. For the exploratory learner this may be fine. Yet for those users apprehensive about computer use, not having sufficient program information might be frustrating. Another feature missing is not knowing where one is in the program, or which unit one has just been in. A student exiting out and coming back in could not know where they have been if they do not write it down. Although each disc has only six units, being able to know the location one was just in would facilitate moving to the next unit without wasting unnecessary energy.

The feedback for incorrect answers seldom gives constructive help. Responses are of the "Please try again" type or "No, that's not correct." Visual reinforcement comes in the form of a red X. After usually two wrong responses the correct answer is given and the program continues. Once in a while the program will say "Let's listen again." A first thought is that the program will repeat the material so that the student can listen again for the answer; instead, only the question is repeated. Feedback, 
however, is immediate for both correct and incorrect answers. The only exercise that allows students to change their responses before being judged is the dictation exercise.

Visuals (graphics) are used in the program, but most of them are vague and useless. Never are visuals referred to for the purpose of learning strategies (i.e. using the pictures to help answer the questions). Many of the practice activities that have pictures on the screen do not need to have the visuals there. An example of the visuals in use is a "\$" representing "bought" and "sold" (this symbol appears on the screen when these words at separate occasions are used). With this example, students can't make a direct association with the symbol because it is used in two totally separate connotations. An X with arrows at each end represents "is located." And very confusingly, a circle with a slash through it is used to represent "lost confidence in"; it is also shown with a house underneath the same symbol when the program says "No housing will be provided." How much the students are able to associate the visuals with the story being told will decide the degree of usefulness. Maybe some students can directly associate the symbols and then use them for schema raising when the symbols appear later in the program. However, using one symbol to represent different concepts can become confusing for students. In general, attention is seldom drawn to the visuals; the program is aurally based.

The activities truly support a drill and practice orientation. Language use is not the focus; rather, discrete segments of language are recognized and used. The program's study guide states “completion of all six discs will prepare you to understand and present basic business information and ideas that are important in a wide-variety of business situations." The program never allows students the opportunity to use the language. The speech examples always refer to someone else; there are no instances of when and how to use the first person. The accompanying literature also states that by repeated listening, students can then talk about themselves 
in a variety of situations. Yet again, there is not any practice, not even multiple choice questions for role-playing activities.

Culturally there is no opportunity for the user to learn or experience culture upclose. It is unfortunate, for business environments by their very nature have a monetarily vested interest in successful communication. Knowing the (business) culture and the possible areas of miscommunication brought about by culture can facilitate cross-cultural communication. There is a potential lead into cultural exploration in the unit about an overseas position, yet it is ignored. A couple of questions appear and ask the user if they would ever consider marrying a foreigner. A "Yes" response prompts the computer to reply "Yes, they can work out very well, but please consider it carefully." A "No" response receives "I see, international marriages can be very difficult." An "I'm not sure" choice receives "Yes, it is a difficult question." The program gives the student its assumptions without allowing the student to answer back or learn more about the reasons behind the computer's responses.

\section{Program Instructional Strengths}

Overall, the program does not take advantage of the computer's instructional strengths. The provision of authentic language use in a variety of situations, or even authentic situations through photos or more realistic artwork is next to none. If people outside of the classroom only speak about others (i.e. use the third person) and not about themselves, then the program is sufficient. Yet students do need to express information about themselves, especially within a business environment. This program does not provide the opportunities to do so.

The program does engage the learner through the use of questions and prompts, yet the forms of interaction are unstimulating after repeated exposure. Any student 
who has experience with the computer can surely become bored with the clicking on single buttons found in the repetitive and decontextualized activities. Feedback is always immediate, which is good, as it lets users know their input was acknowledged, but the input is not helpful or reinforcing.

The best advantage is the inclusion of the recording feature. With proper use it can help students with pronunciation and practice. Yet as mentioned earlier this feature is not incorporated into the activities of the program. The shuffler is also a feature taking advantage of instructional strengths based on the provision of individualized instruction. The shuffler is meant to respond to varying student input, and provide different levels of difficulty. However, any real changes other than a couple of more in-depth questions (the literature spoke of new vocabulary, new comprehension questions, and even new characters appearing to expand the depth of instruction) are not noticeable. Students are also able to decide their own route through the program; they can choose where to start their instruction, but there are no branches for further inquiry or study. Once through a section, the linear path is again replicated by starting another lesson/unit.

\section{Program Recommendations}

I would recommend this program to those who want listening practice and the opportunity for comprehension as well as decontextualized grammatical practice. However, the audio is scripted, so there is no authentic language taking place. Besides, the focus of the listening is based on the recollection of tiny facts, not on the use of business concepts or business English. It is a drill and practice program, intent on producing speakers who can use the language in theory; in practice the program only demonstrates fragmented language use and decontextualized linguistic segments. 
Dynamic English

(DynEd International)

\section{$\underline{\text { Program Description }}$}

Dynamic English is a four level series, although at the time of the review only three levels were available. Levels represented are Beginners (Dynamic English I), Basic (Dynamic English II), and Upper Basic (Dynamic English III). The intended age of user is college/adult. Consisting of a package of two discs, each level presents core information (vocabulary and topics) to be used in the various exercises constituting the lessons of the discs. Comprehension questions are found in the presentation of the core material. The lessons are: "Questions," where students click on the correct ordering of pictures or words and then hear the completed questions with accompanying answers; "Dictations," which are fill-in-the-blank activities of selecting a word to complete the sentence; "Matrix Vocabulary," which presents indepth vocabulary accompanied by visuals; and "Matrix Game," a timed game based on the Matrix Vocabulary. Three lessons have a Speech Recognition (SR) feature: "Fill-Ins," where students fill-in the missing word from the content sentences that can be competed either by clicking on the word, or reading the entire sentence with the correct word filled in; "Questions Practice," which allows students to either make the questions orally using the SR feature or by selecting (clicking on) the correct sequence of words; "Speech Practice," which provides a series of fill-in-the-blank or word reordering activities where students must use the speech feature to progress through the section.

A shuffler feature, which can also be adjusted manually, will modify the presentation of material according to the student's responses. For example, if the student's responses are accurate the shuffler level increases. A speech record/playback 
feature allows students the chance to record and playback their speech at any time in the program.

\section{Program Strengths and Weaknesses}

The Dynamic English series has as one of its strengths a clarity of speech presentation. Students can hear clear, concise English. However, the speech is scripted and provides no authentic input. Students can also practice their own speech by using the record/playback feature at any time in the program. Unfortunately this feature is not incorporated into any activities. Students must on their own use the device. Interactivity is maintained through the use of frequent questions in the presentation units, the supply of questions in the practice units, and by the use of prompts asking if the student is still working on the problem or if the student would like to try the activity or section again. The timed activities prompt the learner to stay alert, although the pause button can stop the clock at any time (the program/activity is easily restarted).

The Speech Recognition feature, available for different levels of recognition (e.g. pronunciation and intonation can be required to be more or less formal), gives students a chance to gauge the pronunciation and intonation of their speech, albeit in limited contexts. The SR feature appears to be programmed to recognize only the specific vocabulary and syntax of the lesson; any divergence from the lesson's vocabulary and syntax then produces feedback meant to show that the computer does not understand.

Feedback is immediate on all three discs, supplying the learner with instant recognition of correct and incorrect answers. In levels I and II the feedback is more constructive for incorrect answers than in level III. Usually responses in all three units consist of "Please try again" or "No, that's wrong." Then the question is repeated 
and the student has another try. Level I, though, has instances (with no consistency) where the information needed to answer the question is replayed, thus giving students at least another chance to glean the needed information. It is perhaps more helpful if the program rephrases the question or rephrases the information relevant to the question. In any case, the replaying of information gives the student another chance. Level II has instances (again, with no consistency) where selecting a wrong answer prompts a computer response telling what the selected picture is and why it is not correct (i.e. "No, that's not a man, that's a woman."). Then the computer proceeds to give the correct answer. In all three levels the correct answer is usually given after two incorrect attempts.

The biggest weakness is the lack of contextual language practice. The program literature states that the design of the program is based on listening. Yet the literature also states that the product "is designed to prepare speakers of English to use the language in a variety of circumstances" and that "after listening extensively to [the] units, students should be able to use the language of the lesson to communicate about their own lives and experiences." First of all, language is used in a variety of circumstances yet learners are never given the chance to practice it. All activities are essentially decontextualized word level fill-in-the-blanks or single sentence repetitions. Secondly, students do not see how to communicate about their own lives because all of the language used in the program keeps the user as an outsider, or an observer, to the language taking place. Learners are not "allowed" into the activities or language because of the use of the third person.

Another weakness is that learners do not know where to begin the program. When the program is opened, students are at a screen listing contents of the program (like a table of contents) yet no indication is given as to where to begin. The available literature states that the units should be accessed one after another, after students first 
look at and understand the content information (all the exercises are then based on the content material). If the learner accesses these activities before becoming acquainted with the content there could be an overwhelming feeling if the exercise content is unfamiliar. While the intentions of the programmers are to give students schema raising and formulating opportunities, a self-study student could be made to feel insecure (compared to one having teacher guidance). If the program were to make a provision of guiding students to the content areas first, negative affective issues could be avoided. Once in the units the learning objectives are not quickly apparent. Although navigation in the program is adequate, students fast-forwarding through the program can not bypass the comprehension questions that appear, even if they have already been answered. Directions of how to proceed overall and within each unit are poor. An exploratory learner might not have a problem if there are no helpful directions; one who feels intimidated, or insecure, may.

True to the behaviorist exercises where there can be only one correct answer, Dynamic English seems to adhere to this principle. Yet Level II does have instances where the program allows multiple answers. That is, there are two answers the program accepts as correct for a question, although allowing a choice of possible answers is not frequent. For example, one sequence in Level II has a scenario where the mother first takes the children to school. Afterwards she goes shopping, and then to the gym. The comprehension question asks "What did she do after she took the kids to school?" Technically the answer can be either "shopping" or "going to the gym" and both are available for selection. Yet the computer does not accept the gym answer. The user receives "Wrong, try again" feedback and is left to make the correct choice. This is an example where feedback could be more constructive. For example a prompt like "She did go to the gym, but what did she do immediately after she took the kids to school?" is friendlier and more helpful. 
While the series uses mainly audio in the presentation of material, visuals are also included. The visuals are adequately helpful in the material. Sometimes they are easy to comprehend, but at other times they are rather vague. For example, an arrow pointing down is used for "to lose weight"; a heart is used for "love"; a circle with a slash through it is placed over a man's face and appears on the screen as the narrator says something about him not being popular with women. Used over a ring, this same circle and slash is used in reference to not being married.

The shuffler represents a good use of the computer's instructional strength in that the feature attempts to provide an individual learning experience based on individual student performance. The identification of the "depth" of instruction available to more proficient students using a higher shuffler level is tenuous. Having an extra-terrestrial make an appearance does not constitute "depth" of instruction although the product literature deems the alien's appearance a feature for students at higher shuffler levels. Granted the alien appearance is attention-getting and a small diversion from the mundane story, but the appearance seems like a one-shot gimmick to keep the student slightly interested and motivated to continue.

Culturally the material in Levels I and II has some bias problems, more so than in Level III. For example, in Level I a man's voice presents most of the information (this could be interpreted as men being more knowledgeable), and men appear to be represented more professionally than women. Social information indirectly transferred through the material includes things like teachers having low pay (students are told that teachers are not rich) and adults can have big expensive homes by not working. Eggs and bacon are shown as a typical breakfast, a sixteen-year-old is shown with a big expensive motorcycle, and messages like "our lives begin at birth" are included. Students are also left with messages like people are put on earth to have children from questions asking "When do people usually have children?"; the user 
also finds out that "most adults get married and have children." While the above examples may represent cultural reality to some people, they may not to others. The program confidently expresses its social views and opinions, and leaves the student to passively play along. Level III has a more neutral outlook in the presentation of material.

\section{Program Instructional Strengths}

Through the shuffler the Dynamic English series attempts to incorporate individual learning paths. As mentioned earlier, interactivity is maintained through the use of questions and prompts, yet it is mechanical and uncompromising. Feedback is rarely facilitative, although it is immediate. The sources of input (text, audio, and visuals) are varied but the focus is blatantly on the audio component. Tasks are repetitive and uncreative, and students are never given the chance to practice communicative language, or examine culture.

\section{Program Recommendations}

I would recommend this program to those unfamiliar with the potential of computer software products, yet having some familiarity with the computer. The program offers a good source of aural input and comprehension-based practice, yet the activities seem to promote passive learning and the language students are expected to learn does not represent the language used in realistic interactions away from the computer. 
Let's Go

(DynEd International)

\section{Program Description}

To be used with children who are first time English learners, Let's Go is a two level program for individual, group, or classroom use. Each level, 1 and 2, consists of eight units on two CD-ROMs. Each of the eight units is composed of an introductory song and dialogue indicating the unit's topic, vocabulary work, a grammar exercise, and a phonics exercise. A game to practice the lesson's content is also included. Let's Go uses audio, text, visuals, and the animation of two cats, Sam and Ginger, to provide students with language material functionally-based around situations the student may encounter. Language functions like introductions, greetings, describing objects, and expressing likes and dislikes are presented through a number of tasks. In the vocabulary based exercise, students see and hear vocabulary by clicking on various visuals. If the vocabulary contains verbs, animation supplies the learner with another form of visual reference. Students are then asked to record their voices so that they can compare their pronunciation with that of the program. Afterwards a small scored quiz checks comprehension. The grammar activity has students responding to Sam's questions by clicking on supplied choices. Ginger appears on the screen to give the students feedback for both correct and incorrect answers. Users can see and hear the letter names and corresponding sounds in the phonics section. The phonics exercise has students listening to determine the sound they are hearing and then selecting the correct answer. The games are different for each unit. Most games rely on multiplechoice formats.

In Let's Go, students can practice listening, speaking, and reading (students can record their voice at any time in the program beyond the vocabulary activity). 
Bilingual translations are available, and students can repeat activities and information as many times as needed. Record keeping is available for individual learners. The program is based on a series of the same name. The accompanying literature states that the software can be used as a stand alone program or in conjunction with the series that contains a text, workbook, cassette, and picture cards.

\section{Program Strengths and Weaknesses}

Let's Go, although essentially a linear tutorial-type program, does a good job in actively engaging the learner. Students are asked for their opinions and likes/dislikes, they can ask the cat questions who then answers them back, and they are textually greeted by their name when they log into the program. Right from the beginning of the program, students are informed visually and aurally of the different buttons available to them (e.g. the cat points out "this is the listening button, this is the reading button"), and they are for the most part led and reassured throughout the program. Students are asked "Which unit do you want?"; they are asked to make a selection to move the program along (instead of being subject to a predetermined path); students are given informed choices (a cat tells them to "Click here to learn new words"); they are told how to activate the exercise if they spend too much time doing nothing at the screen; students are shown the navigation tools that will easily move them from screen to screen in both forward and backward directions.

Language is presented in an animated context. The two cats act as friendly supporters who offer friendly feedback and help to the student. Although language is presented from non-human characters (and therefore does not provide a realistic human-to-human context of communication), the language samples that are given provide the students with functional language used in realistic settings. For example, in Level 1, students see formal and informal language use when being introduced to a 
grown up and another child. Although the program does not outwardly bring the child's attention to this sociolinguistic difference, the child can still experience it through the animated visuals. At other times the user can see situations that might warrant a type of sociolinguistic behavior. For example, a cat sitting sadly on a chair has another character asking "What's wrong?" Although there may be some cultural bias based on the showing of emotions (different cultures show emotions differently, and thus have different expectations of other culture members), the student is given a look at culture and appropriate behavior within a given context.

The combination of media (text, audio, and visuals with animation) works well together and provides clear input for the different learning styles. Visuals are engaging, and active when animation is used. There are some interesting characters in the games, occasionally showing up as feedback, that help maintain the user's attention. Consistency is maintained by the types of interactivity (usually clicking on objects in multiple choice type activities), and the icons used are simple and clear.

Feedback is constructive for both correct and incorrect answers (although more so with correct answers). Correct answers have the program responding by repeating the selected answer, or providing positive reinforcement of why the other choice is not correct (i.e. "That's right, it's not a pencil, it's a pen."). For incorrect answers the feedback consists of reinforcement of the answer chosen (i.e. "No, it isn't a pencil, what is it?"), or friendly responses like "Are you sure? Try again." If the student needs to do multiple actions to answer a question (i.e. click on two items instead of one) and starts off by selecting an item out of sequence, Ginger appears and points to the general section needing to be clicked on first and says "Start here." In this case, students are given the opportunity to try again without immediate feedback like "You're wrong." The program recognizes the apparent complexity of the exercise 
and does not penalize the student who may not understand the routine on the first attempt.

The biggest weakness is that students are not allowed too many opportunities for language use; the program tends still to focus on form. There seems to be a better, albeit small, attempt in Level 2 to allow more personalization by allowing the student to ask a question that is not fully predetermined: the student have the choice of asking "Who are they," "Who is he" or "Who is she" as two characters became visible through the bus-window at a bus stop. Based on the student's interest of wanting to know either who both of the characters are, or who individually each is, the student has a choice and the feedback gives the appropriate answer to the student's question.

Another shortcoming is that the program is so user-friendly the student might let the program do all the work. If a student sits long enough without any activity, the program guidance appears and does the work or gives the answer. The student can then become over reliant on the help feature, thus becoming a passive learner. On the other hand, some students may need to have this guidance. Perhaps if the program had better record keeping capabilities it could recognize when a student was floundering or just misusing the help that is available.

\section{Program Instructional Strengths}

The Let's Go series does a good job of integrating the various media into a package for various types of learners. The shuffler feature is also used to provide a more individualized instructional path based on the user's input. The program provides learner interaction through a variety of tasks that, although consistently the same and behaviorally oriented, are engaging and fun to do. Feedback is immediate and helpful, and students are able to see language used in a variety of realistic situations (albeit conducted between non-human characters). As far as providing 
culture and up-close experiences of culture learning, the program does not make any concessions. While the program adequately "provides the vocabulary and language structures needed for everyday communication" as written in the product literature, students are not given the opportunity to actually communicate at a level found in everyday communication.

\section{Program Recommendations}

I would recommend Let's Go to those the program was designed for: first-time child learners. The atmosphere provided through the material is friendly and supportive. Situations of language use typical for children provide the user a chance to practice some language, even if the focus is mainly on linguistic form.

\section{Learn to Speak English}

(The Learning Company)

\section{$\underline{\text { Program Description }}$}

Learn to Speak English is a two disc program for Advanced Beginner/Intermediate Level students. Using text, audio, visuals, animation, and video, the student encounters thirty chapters sequenced for the telling of a story/adventure of a woman who arrives in America to carry out the wishes of her deceased Aunt before being able to claim the inheritance. Each of the chapters is then based on a situation the woman encounters on her journey (i.e. immigration and customs, checking into a hotel, renting an apartment, going to a lawyer, getting caught in a traffic jam). Beginning each of the chapters is a screen stating the communicative goals of the chapter. The chapters are composed of units made up of vocabulary, 
information outlining the "story" and the "action" taking place, listening skills, a number of practice exercises, communicative skills, and a selection of games.

In the vocabulary unit line drawings, text, vocabulary notes, and audio present the words used in the chapter. Students choose vocabulary located on a scrolled list and are immediately given the visual representation and pronunciation of the item. A record feature and a pronunciation gauge are available for the students to practice their own pronunciation of the word. Students can also see and hear the vocabulary word as it is used in the context of the situation presented in the chapter. The "story" screen is where students can play the video and see a native speaker use the language; the text of the script can be either present or not, as students so desire. The "action" screen is similar to the "story" screen in that the video is again made available. On this screen students can focus on the dialogue taking place, either line by line, or in its entirety. Cultural notes can be accessed, and students can record their voices, even using the video as gauge for their rate of fluency (although students' rate of accuracy is not determined).

In the listening skills section students must type the word they hear in the correct blank to complete the written dialogue. If the student's answer is correct the matching video clip runs. Other activities found in the Learn to Speak English program are fill-in-the-blank type exercises based on vocabulary and grammatical constructions, and a click-and-drag-based activity where students put a jumbled sentence back into the order of a syntactically accurate sentence. The unit of communication skills has students listening to or reading various situations. Students must then respond, either orally or by typing, and accurately supply the missing string of words. This exercise is scored. Finally, a number of activities like Go Fish, crossword puzzles, and matching exercises compose the game unit of the chapter. 
The program has many features. As mentioned above, a record/playback feature is available along with a speech recognition gauge that can be set at various levels of exactness (i.e. from more native-like to less native-like). Students also have control of many options. For example the text can be displayed or not, the speech recognition gauge can be set, the time allowed for recording can be shortened and lengthened, and animation can be turned off. Through the video clips students see language use by individuals; the story taking place with the numerous situations allows a look at the different contexts of language use. The availability of cultural notes gives background information regarding the situation. The inclusion of cultural movies showing the major cities across the United States also adds a degree of cultural awareness by presenting some history and tourist-oriented aspects to the geographic locations. The skills of speaking, writing, and listening can be practiced through the activities. An accompanying program based on English pronunciation is also included on the discs.

Learn to Speak English is accompanied by a combined text and workbook that can be used for study away from the computer. The text contains supporting exercises different from what is found on the program. A User's Guide also accompanies the program. In it all the features and instructions/directions for the use of the program are available.

\section{Program Strengths and Weaknesses}

Perhaps the largest strength of Learn to Speak English is its presentation of realistic sociolinguistic situations (though perhaps not encountered by all English learners) with cultural notes so that students can understand the context of language use. If the situations had been set in authentic settings (i.e. at a real hotel) instead of filmed in a studio, students would have a better opportunity to get close to more 
realistic situation for language use. However, students can still see how a foreigner might reply to the seemingly over-friendly nature of restaurant staff-people or how to react when an American stranger trying to be friendly asks a foreigner to dinner (accompanying cultural notes tell the learner that "it's not unusual [in America] to receive a dinner invitation from someone you barely know").

The communication skills unit offers students the chance to try out their knowledge of the linguistic formulas needed to respond to various situations and questions. For example, a written prompt appears with "You go to a bank to change some money. You say...." Here students either type or speak the correct response (the expected response is something like "I want to change some money."). The speech recognition feature is one that recognizes key words (compared to one that can only recognize exact strings of words/sounds), so the student is allowed some flexibility in the spoken responses. However, even saying an inaccurate grammatical response that includes the key words results in feedback saying the student is correct. As much as this handling of speech can be attributed to the shortcomings of technology, the student is misled into thinking the response is correct. Written responses, on the other hand, must be 100 percent accurate in spelling, grammar, and punctuation.

Being able to choose whether to have features like graphics, instructions, and other program features present offers a feeling of customizability to the program. On the "Preferences" screen students can customize recording length time, the availability of text, the inclusion of the opening video sequence, the table of contents to a grammatical or situational ordering, the level of speech recognition (strict, normal, relaxed, or make it inactive), and they can bypass the "Welcome to" greetings accompanying each unit, as well as turn off the animation that, although in most cases lively and interesting, can soon become repetitive. So many preferences provide the 
student with some control over the presentation of material to individualize the experience of the program.

The accompanying book offers reinforcement of the program's content through additional exercises, but that is assuming students will use the text. Not only does the text offer supplementary activities, it also addresses many issues like learning strategies (it tells the reader to "use the drawn images associated with new vocabulary words as much as possible" and to use the recording feature as much as possible) and how to best proceed through the software program. Unfortunately for those who will never read through the text (maybe they are unable to because of the complexity of language used or perhaps the book becomes lost) this sort of information is not made available any other way. For example, the program uses pen and ink drawings in the vocabulary section, but students must learn by themselves either intuitively or having been previously shown, how to associate the graphics with the contextual lexical referent. And students are left to decide the best path through the units in each chapter, without possibly ever knowing they are expected to follow the preferred sequencing.

Another weakness of the program is its often weak representation of vocabulary by the accompanying drawings. Concrete objects such as cheese and bread are easily represented. Yet more abstract associations get lost. For example, the visual representation of the verb "bring," showing an outstretched hand and another hand holding an ice-cream cone, seems to better represent the verb "give"; the hands shown to represent "beg" looks more like they are praying; "certainly" is shown by a speech-bubble containing exclamation marks; "complimentary" is meant to be associated with a collection of five quivering exclamation points. Although the interpretation of the drawings might seem adequate to some, others may have some difficulty in making the correct association. 
Beyond the vague visual representation of some of the vocabulary, students in the vocabulary section also have no access to practicing the different words. Students need an opportunity to apply the words in contextual uses. It is not enough to see the written form, see the associated visual, and then hear the word used in context, especially when the contextual use of the word in the video often does not match up with the corresponding definition. Consider the word "cheese." A slice of cheese is shown as the accompanying visual; it is easily identifiable. When the student decides to hear the word used in context, the video refers to "cheesecake." Imagine the nonnative speaker unfamiliar with cheesecake who now thinks a common table-type cheese is used in its making (this could create an interesting cheesecake if the learner only uses the visual representation of cheese when in the store shopping -- of course this assumes the learner will even feel compelled to make a cheesecake). To learn new words the text recommends that students simply repeat the words often enough until the words become memorized.

Perhaps the greatest weakness of the program is its lack of activities where students use language beyond one word responses or strings of words that match the required linguistic formulas used in the various situations. It is unfortunate, for the program supplies a wealth of sociolinguistic and cultural information that can be used yet the exercises never progress into ongoing exchanges characteristic of conversations. It is a strength that students get to orally practice speech, but students always play the same part. The communication exercise offered in the program conditions students to a number of situations, but always by using the same sort of language and linguistic formulas. Branching stories might provide some divergence, so that students might encounter unrehearsed language use. 
$\underline{\text { Program Instructional Strengths }}$

With regard to how Learn to Speak English uses the computer's instructional strengths, the program uses some and leaves others out. For example, the program does provide examples of contextual language use for the student to see language used in a variety of situations, but the program does not allow the student to use language beyond the required linguistic strings or one-word responses. Feedback is immediate, letting students know that their responses were registered by the computer, but feedback is not always helpful when students are incorrect, especially the typing feedback. The use of the recording and playback feature is a thoughtful way to exploit the potential of technology, but students must feel compelled to access the feature on their own. Cultural information is presented and helpful to the understanding of the language use, but students are not given the opportunity to compare that culture with their own. The application of individualized instruction is strong for this program, although the path of instruction is fully linear and predetermined, with no chance for the more curious student to learn more about a specific area. The use of the different media (video, text, audio, and drawings) does supply the learner with various sources of input that can also be customized based on learner preferences.

\section{$\underline{\text { Program Recommendations }}$}

I would recommend this program to individuals who desire to learn about a number of situations and basic associated language use common to tourists and shortterm residents in the United States. However, the scope of situations can easily be applied to those learners spending more than two weeks in the USA. The inclusion of films of various cities also supports the recommendation of this program as one for tourists, for the films seem like tourist advertisements (hopefully not too many tourists will recognize that the Chrysler Building in New York City is actually the Empire 
State Building) and not culture-learning documentaries. This program is good for limited pronunciation practice, and for becoming cognizant of various linguistic formulas.

\section{The Rosetta Stone, English, Levels I and II} (Fairfield Language Technologies)

\section{Program Description}

The Rosetta Stone English program contains two different levels (Level I and Level II) for the learning of words and basic grammar. Both levels are designed for ages eight to sixty. Level I is intended for Beginners to reach an Intermediate level. Level II is intended for Intermediate learners to reach a more Advanced level. The content of the program refers to a number of grammatical/structural (i.e. nouns, prepositions) and notional (i.e. time, shapes and colors, quantities) categories. The lexical items presented in the beginning of the series are sequentially built upon to larger chunks of languages (i.e. sentences). Some discourse level language is also available.

Each level is made up of units (Level I covers units one through eight, II covers units nine through nineteen) comprised of photo images, text, audio, some video, and drawings. The chapters of each of the units contain ten screens of four photos where students can begin to associate words with real-world objects. A review chapter is available at the end of each unit. The activities use a multiple choice questioning format based on the number of different "Run Modes." Each of the twelve Run Modes allows the student a choice of content presentation. For example, Run Mode 1 uses audio and text to present the lexical information; students then select the correct photo (a choice of four) that corresponds to what is seen and heard. Run 
Mode 2 uses only audio and the students must then associate the spoken word with one of the four photos. Each of the Run Modes uses a combination of text, audio, and photographic imagery to engage the student in the exercise.

A tutorial provides three different options for students wishing more guided practice in the program. (All three options contain the photographic images.) One option is based on the Run Modes containing audio and text; another uses only audio; the last option is a random assortment of activities based on a variety of the Run Modes. Dictation practice found in the program has students typing the associated meaning of each of the four pictures after first hearing the photo's description. Finally, a "Browser" gives students access to the chapter's content before accessing the activities available in the different run modes.

Besides being given the choice of the presentation of content through the various Run Modes, students also control the volume, the type of feedback given (both sound effect and visual; feedback can also be disengaged), whether or not the activity is timed (as well as the length of the time interval), and whether or not an activity is a test (in this mode Help features otherwise made available are disengaged). A record/playback feature in the Browser Mode lets students record their voices to compare with the native speakers used in the presentation of material.

The Rosetta Stone program is not used in conjunction with a text. A student workbook for Level I is available, and a handbook for teachers can be used for both Levels. Accompanying information for both Levels provides the text of the program and some exercises. As a separate program, a student management system is available to keep track of student records. 
Program Strengths and Weaknesses

The quality and accurate content of the photographic images provides the user with easy-to-understand visuals. The richness of the photos presents the user with a direct visual association of the content (individual lexical items as well as sentences/descriptions). The broad representation of content provides useful language that, once learned, can be applied to a number of situations. There is also a good representation of different ethnic backgrounds in those photos containing humans. The biggest instructional strength of the program is the availability of the twelve Run Modes. Students can choose the best Run Mode to fit their learning style (e.g. if students prefer to learn by listening, they can pick the Run Modes that focus on listening, if students prefer reading, or visual learning, they can choose the Run Modes that focus on those areas). Run Modes can also be used to practice the skill areas (reading, writing, listening) students require more help in; the Modes can also provide different levels of difficulty.

Sociolinguistic language use is presented, although it is not the focus of the program. However, students are still given small samples of language use addressing topics like formal and informal language, greetings and conversations, social conventions, asking for information/clarification, and transactions at a bank (Level II has a better representation of contexts for language use with common social conventions, greetings and conversations, telephone calls, and making inquiries and requests as typical chapter material). Although students are given these samples, they are not given the opportunity to practice the language. In the units addressing language functions the practice students do get is fragmented. No continuity of the linguistic transaction can be experienced. For example in Level II (Unit Ten, Chapter two) common social conventions are addressed. Screen five contains the typical fourphoto layout. Each photo clearly represents its associated linguistic referent. One 
photo represents "No, thank you." Another represents "Yes, please." The third is for "May I help you?" And the fourth is "Excuse me, can you help me?" When it comes time for the student to do an exercise, the same multiple choice format appears in whichever Run Mode chosen and the student is never given the chance to see how to sequence the language use that transpires in the common social conventions the chapter addresses (i.e. a sequence of the material could be the initial "May I help you?" The reply could then be "No, thank you."). Most of the language presented is in reference to other people (i.e. "The boy is jumping."); seldom does the content offer information to be used in the first person.

Another program weakness is the repetitive nature of the multiple choice activities providing no real goal other than the completion of the exercise (which is basically to associate the photo with its lexical referent). While language learning, both first and second, involves a degree of repetition of linguistic structures and vocabulary, language use needs to be based on the desire and need to successfully complete a (linguistic) transaction. Students are not provided with this sort of end result; they can only repeat and repeat the content devoid of any real-world transactions. The feedback used is also a weak component of the program. While it is immediate, the feedback for wrong answers only makes the user aware that an incorrect choice is made. The user can then answer incorrectly (i.e. try out all the given choices) until the correct answer is selected. This can promote passive learning.

Although the material presents engaging photos showing different ethnic backgrounds and cultural artifacts, the cultural information available can be presumptuous and judgmental, especially as it relates to gender. For example, one sees a man with shorter hair and learns that his hair length is the usual length for a man. Other information shows a woman cooking at home, but a man shown is cooking as a chef. A woman is also shown as one who does all the clerical work in an 
office (licking stamps, filing, weighing packages). At times the program provides more unbiased material (for example, a woman working on her car is shown, and a man doing his own ironing), yet there are still some messages that can perpetuate gender differences (for example, a father is proud of his son, not of his daughter; a boy can be disobedient but his sister obeys) that remain unexamined. The handbook for teachers addresses cultural activities that can also be extended to those looking at gender issues, but there are no activities for culture learning in the programs.

\section{Program Instructional Strengths}

The program takes good advantage of the computer's instructional strengths through the incorporation of the twelve different Run Modes. The opportunity for individualized instruction is fairly strong for this program. Students are provided varying sources of input (photos, audio, text, drawings) that can be tailored to student needs or used for levels of difficulty. Task types are not too varied; multiple choice using the different sources of input are the staple, supplemented with dictation exercises. The recording feature provides another source of task type, yet this feature is not integrated into the activities. Interaction then is minimal and there are no prompts to actively engage the student. Examples of communicative language use are fragmented, and students are not given the chance to use the language beyond the decontextualized environment it appears in. Culture examination is not addressed in this program.

\section{Program Recommendations}

I would recommend this program to those not overly familiar with the instructional potential of the computer who desire a repetitive practice of vocabulary and linguistic structures covering a variety of topics. Actual language use is not the 
focus of this program, yet the rich visuals provide the user with accurate and dramatic visual referents that can make any vocabulary learning exercise enjoyable.

TriplePlayPlus!

(Syracuse Language Systems)

\section{Program Description}

Intended for users age eight to adult having Beginner to Intermediate level abilities, this single-disc software package offers twenty-one games and twelve comicstrip type conversations for users to learn English at the word, phrase, or conversation level. After choosing one of the three modes of running the program (reading, listening, speaking) and the area of interest (food, numbers, home and office, place and transportation, people and clothing, activities) the user can then choose any one of the games or conversations available for the mode and interest levels selected (not all games and conversations are available for every mode and interest combination). Many of the games have levels of difficulty available. Two levels of games provide linguistic content at the word or phrase level. The conversations provide discourse level material.

Level I games allow students practice with word level vocabulary that make up each of the interest areas. Games use a multiple-choice format, with some fill-in-theblank, and a heavy reliance on memorization of material which is available for previewing before the game takes place. Examples of Level I games are "Concentration," where learners respond to either an oral or written clue (depending on whether the learner is in the listening or reading mode, respectively) by selecting an item whose identity is concealed like a face-down card (students are expected to memorize the items and their location before playing the game); "Square Off," where 
students hear a word and then select from a number of items displayed on a grid, with the goal being to make more squares (every correct response lets the user build another side to the square) than the opponent (the opponent can be either the computer or another student). Speech Recognition Technology (SRT) is available for some of the activities (students must first select the speaking mode to access this feature). Level I activities with SRT has students speaking the individual words needed to complete the games (for example in "Bingo" students must say the word on the grid that the arrow is pointing to, instead of just clicking on the picture from the written or pictorial clue when SRT is not used).

Level II games present language beyond a one-word level. Most of the games use descriptions of the vocabulary items (for example in "What food is it?" students hear clues like "They're brown, they grow in the ground, they have a shell" and are expected to choose the peanut seen on the grid containing an assortment of pictures) instead of the one-word referents found in Level I games. SRT is also used in Level II games. In the "What food is it?" game students must speak the answer instead of selecting it.

Level III activities contain twelve dialogues presented with characters in a comic-strip type setting, complete with speech bubbles. Topics of conversation can be broken down into the six subject areas of the program (e.g., food, numbers, home and office, etc.). For the "Food" subject area the conversations contain dialogues at a café, and at a restaurant'; For "People and clothes" the dialogues represent language used when shopping for clothes or going to the library. The conversations are available in all three modes of practice. Students can practice the conversations role by role, and even practice individual words if that sort of practice is needed. Using SRT students use speech to respond to what was said (written choices are given) in the dialogue. 
As mentioned earlier, TriplePlayPlus! gives the students a choice of the mode of material presentation and practice, as well as a choice of any number of games to practice with. Almost all of the games allow the student a chance to become acquainted with the content material before practice (playing the game) begins. The SRT feature can be set so that user voice type (i.e. male, female, child) can be better recognized by the program. Record and playback features found in many of the level games/conversations provide speech practice that can be compared to the native speakers used in the program. No textbook is used with the program.

\section{Program Strengths and Weaknesses}

The available modes (reading, listening, speaking) along with the subject area and the assortment of activities allow users to have more of an active role in making decisions about their learning wants and needs. Students do not just enter into the program and progress along a predetermined path. Instead, choices are available. Not only are there options for the mode and subject area, but many of the games contain varying levels of difficulty that students can also select. The different levels of games allow students a choice of word, phrase, or conversational level language, depending on the needs and wants of the student. Some branching of information is used in the Level III activities. This information provides more examples of specific language use. For example, in one dialogue "May I help you?" is used. The branching information provides other contexts "May I help you?" can be used in (the program shows how it is used in a department store, and with a bellman).

Some of the feedback is beyond the "Outstanding " and "Wonderful" or the "Bad luck, try again" and "No, that's not correct" usually supplied. For example, one activity has the computer asking the student to identify "the girl that's reading" from a number of other children engaged in various activities. A wrong answer 
receives "No, that's the boy that's drinking. Where's the girl that's reading?" The user is then allowed as many opportunities as needed to find "the girl that's reading." Another activity has feedback saying "No, that's the yogurt. Find the cake," when the answer selected is incorrect. A frowning face accompanies almost all negative feedback, yet the face changes to the smiling face when the clue is repeated. The change of the frowning face to the smiling face can leave the user feeling more comfortable than if only a frown is used.

TriplePlayPlus! provides a variety of games, yet the directions given are extremely verbose and beyond the Beginner/Intermediate learner. Even though the learner can get access to directions by using the F1 key, and even though the scrolled list will automatically take the student to the section needed on the list (i.e. students do not need to scroll to find the directions for the activity they are in), the directions are by no means easy to understand. Some examples of how the game progresses, as well as clear goals of the game (i.e. how to win) would make the directions better. This leads to another weakness of the program: its use of games purported to be "popular" and "familiar" (e.g., "Concentration," "Bingo," and "Square Off!"). While there may be some games that transcend cultural boundaries, one culture's interpretation of the game may be different from another's. Therefore games intended for use by people of different cultural backgrounds need to show the goal of the game. It cannot be assumed that what is popular and familiar in one culture is so in another. The use of games is not the only case of an ethnocentric bias: a picture of the United States is shown to represent a "World Map."

Another weakness stemming from use of games is the questionable appeal to the user. This program is intended for Beginner/Intermediate users from the ages of eight to adult. However, many of the dialogues in Level III activities are not relevant to an eight-year-old. Looking for an apartment and shopping for food are not typical 
contexts young people find themselves in, and some branching information provides information not relevant to adults (for example, branching information for " What's your name?" in the library conversation module shows "I'm Papa Bear" and "I'm Mama bear" as other answers one can use). Though it is practical that some immigrant children may find themselves as the link/contact for their family between the new culture and language, the dialogues provided may be impractical for most children. This is unfortunate, for the Level III dialogues are the only activities offering examples of language use at the discourse level.

The dialogues supply the user with realistic language use for a number of situations, but the comic-like setting takes away the meaningful situational quality of the discourse taking place. Some of the contexts also provide the learner with inaccurate sociocultural information. For example, the dialogue about being in a café shows a woman sitting at the counter with a small dog. This may lead some to believe it is all right to take a pet into an eating establishment. Another dialogue taking place in the library has two strangers meeting (a muscular American male and a petite Italian woman). The opening line is "Hi, I'm Ken. Where are you from?" The woman answers back and in the end the two decide to go on a date. This example, besides showing a very forward use of language, provides the message that strangers can be trusted. Therefore, even though students may be learning the correct sociolinguistic use of language, they are not supplied with accurate background information (i.e. it is not always safe to go on a date with strangers) to aid them in making the decision of continuing the interaction or not.

The appeal of the activities for the user can also be questioned in reference to some of the vocabulary games intended for adults. Many of the games are devices meant for the practice of vocabulary. Depending on the background of the adult, one more educated can find these games demeaning and childish. Although different 
levels of difficulty are provided within many of the games, adults may soon tire of the repetitive and simplistic nature of the activities.

With so many games one might expect some sort of record keeping to keep track of what was already accomplished; with the different modes of learning, different content areas, and different games, one can easily forget which combination and what games were already completed, and at what level of difficulty. The use of records allows the user to assess and gauge the progress taking place. Records can also provide students with motivation as students attempt to beat previous scores.

\section{Program Instructional Strengths}

TriplePlayPlus! provides the user with choices that can be used for more individualized instruction. Students are not only able to choose a mode for learning, a content area, and a game, but also the language level (word, phrase, discourse) to be practiced. The different modes of learning provide various types of input delivered through visuals, text, or audio. Different levels of difficulty in some of the games are provided; the different modes can also be used for difficulty levels. Feedback is immediate for all student responses, with some feedback going beyond the "wrong try again" and providing more helpful information to the student. The program does not use prompts to engage the learner or to help the learner struggling with the program; the learner is left to figure out the difficulties alone. Examples of realistic language use in a variety of situations are provided, and the associated activities provide the student with the opportunity to partake in predetermined and routinized conversational exchanges. However, background information about the context of language use is minimal, and students are not given the chance to actively examine sociolinguistic language use and the culture within which it is found. 


\section{Program recommendations}

I would recommend this program to users not from the age of eight to adult, but for those users who are teenagers to young adults (early to mid twenties); the presentation of content does not seem realistic to most users over age thirty. The user needs to be comfortable with exploring the program as there is no real guidance with how to complete the games. The user should also be one still enchanted by the computer; anyone used to more advanced programming can easily get bored by the simplistic quality. TriplePlayPlus! provides restricted language practice for a small assortment of subject areas; anyone desiring mechanical listening, reading, and some speech practice offered through a variety of games and dialogues might enjoy this program.

\section{ELLIS Senior Mastery}

(CALI, Inc.)

\section{$\underline{\text { Program Description }}$}

Delivering the core of its content by the use of video, ELLIS Senior Mastery is for users over fifteen years of age at an Intermediate to Advanced skill level. The single-disc program can be used for higher-education, business, or high school settings. Students using this program can see a variety of language situations and receive information and practice regarding vocabulary, culture (including sociolinguistic expressions and sociocultural behavior), grammar, and pronunciation. Students can practice speaking at a discourse level through the use of a role-play activity.

The core of the program consists of six main content areas or categories (academic and non-academic survival and communication skills, short and long term 
budgeting) that are divided into twelve video-based lessons. Each of the lessons contains modules that are essentially the breakdown of the video into smaller segments. For example, the Non-Academic Survival Skills category has four lessons ("Getting a Driver's License," "Shopping at a Convenience Store," "Job Hunting," "Technical Discussion"); each of the lessons then contains modules ("Job Hunting" has three modules: "Reading want ads in a local newspaper," "Calling for information about a job," "Calling to set up a job interview"). Besides the "Conversations" section the program is also organized by "Conversations with choices" and "Conversations by grammar topic." The former, through the inclusion of story branches, allows students to determine the outcome of the action taking place in a module. The latter is organized around grammar topics; students can choose a grammatical topic and the video segment using that topic is automatically made available to the student.

Once a module is selected, students can see the entire video sequence to see their module in context, or, they can have access only to the segment chosen. After the running of the clip, a script of the video segment appears on the "Script Page." Here the student has access to the video line by line (simply by clicking on the line in the script); students can also slow down the audio of the film, and also record/playback their voices. Along the bottom of the script page are a number of buttons that provide further investigation into the content of the video. "Culture," "Vocabulary," "Grammar," "Video," and "Phrases" buttons provide the user access to the corresponding examples found in the video segment. For example, if a student chooses "Culture" the "culture" words in the script are highlighted. By clicking on the highlighted box students can access scrolled culture notes. The "Video" button shows the video segment again with the script below it, with key words only, or, simply by itself. A role playing activity, where students choose to be any one of the 
characters in the video and then record their own voice that can be played back as part of the dialogue, is accessed from the "Video" button.

Besides the video units, ELLIS Senior Mastery has "Practice Activities," "Speaking Activities," and "Listening Activities." Practice activities use multiple choice (single or multiple answers accepted), true/false, matching, and fill-in-the-blank questioning formats. Students can select content from vocabulary, culture, or grammar at three different skill levels (easy, medium, and difficult). Speaking activities include a video profile of a man or woman, or, an animated model, showing how the vocal tract works when particular sounds are produced. Students choose a phoneme and see how it is produced; some words using that phoneme can be seen and heard. Students can record their own voices to compare with the example. One of the listening activities use a video segment with comprehension questions, the other is based on minimal pairs. The latter allows students to practice specific phonemes selected by the student, phonemes given at random, or those phonemes identified as troublesome to specific language groups. The "Skill Check" of the program provides a comprehensive test of 100 items covering the lessons, and the "Your Performance Status" shows students the conversations viewed, the performance on practice items, as well as time-on-task information.

In addition to the features discussed above, the program also allows different users (up to three) to log on and work together at one terminal. "Instructor Utilities" for record keeping are also used with the program. Students using the program must first be acknowledged by (i.e. registered with) the instructor utilities so that records can be systematically kept and updated. Besides keeping track of records similar to those available to students on the program, the utilities let the instructor organize the students by name, age, class, native language, or the identification number the student uses to access the program. 
A text is not used with the program, but an Instructor's Guide and a Learner Response Book are available.

\section{$\underline{\text { Program Strengths and Weaknesses }}$}

The treatment of culture, although not fully integrated into the program material or activities (any culture information or practice must be selected by the user, it is not automatically presented), provides the user with some detailed information regarding culture and sociolinguistic language use. The culture notes provide cultural background information to the many contexts of language use shown in the video segments. Most of the cultural information is accurate. Some of it could benefit by revisions or else stand to be considered unrealistic. For example, in the unit about job interviews, the user is left with the impression that just by the filling out of an application an interview is automatically granted. There is also a case where a male aggressively asks who a woman's friend is (it is another woman who is standing right there); perhaps the actor is only exaggerating his part, but the result sounds almost lascivious. Beyond some of the inaccuracies of cultural information, the program does provides the user through the use of the branching stories a look at even more uses of language in a given situation. Students can then share in the initiation of events to determine the outcome of a story.

The role-playing activity provides students the opportunity to practice language for more than one part, instead of playing the same role over and over. Students can record the lines of the dialogue as they take any character guise and then hear themselves as the scenario is played back in its entirety (with the student's voice included). Advanced level imaginative students can even come up with their own lines to fit the dialogue, if they so desire. Students can not only practice the exchange of information that takes place in a dialogue, but also work on their pronunciation, 
phoneme by phoneme; the particular areas identified for specific language groups let the user hone in immediately on possible problematic pronunciation areas. The choice of content presentation (i.e. by grammatical topic, with choices/branches) allows a sort of customization to the program, and the program's record keeping lets students keep track of their learning. The surface features of the program are good; the use of hotspots lets the students know what buttons are active. This use of hotspots can also make the student feel acknowledged by the computer.

With all the information available on the script screen (the script and all the buttons leading to cultural or grammatical information), students are not actively engaged to use it. Instead, a student must access the information alone by either exploring or fulfilling some predetermined task set up by the instructor. Later on this same information is represented in the various activities, yet in what might be thought of as the previewing-of-information screen (i.e. the script screen), the student is a passive participant. Throughout the entire program the student is rarely provided any active engagement. Granted the program allows the student an individual pace through the program, but some sort of acknowledgment when a student pauses at great lengths (possibly signifying confusion or boredom) would create a more user-friendly program. Another user-friendly concern is the lack of any tool so that students can know their location or where they have just been. With so many options available to the student a feature showing the path taken by a student would be helpful. The record keeping does show some of this (e.g. what conversations were previously viewed, etc.) but to even show up on the records the student's progress through the program and activities accessed must first fulfill the predetermined requirements of the records.

While the culture information presented provides some background information regarding the general cultural behavior and sociolinguistic language use of the United States, the examination of culture at a deeper level is not done. This sort of 
examination could provide users with a more direct connection to possible cultural misunderstandings as well as provide users with a chance to examine their own culture and make some comparisons.

Perhaps the biggest weakness of the program is the type of feedback used in the true/false activities. More often than not the feedback is the same for both correct and incorrect answers. As an example consider the feedback for the true/false question "It's not socially acceptable in the U.S.A. for women to ask men for a date." For both true and false selections the following appears: "Just the opposite is true. It is common for women to ask men for a date." Accompanying a correct answer is also a green check that turns pink; a wrong answer gets a red X. Because of the similar feedback for both correct and incorrect responses, the user is not given immediate confirmation of being correct or incorrect. Instead, the user may begin to rely on the behavioristic visual (the green/pink check or the red X) that accompanies the answer and not even have to read the feedback that, although similar for both responses, contains some helpful information. The use of similar feedback found in the earlier activities might even hinder the attention paid to feedback in later activities that is not similar (and thus more constructive). Instead, students can look to the graphic display to determine their accuracy when answering the question.

\section{$\underline{\text { Program instructional strengths }}$}

The use of video provides examples of sociolinguistic language use at the discourse level (albeit scripted examples) in a variety of situations relevant to the intended audience of the program. Communicative language practice is made available through the role-playing activity, and different roles for the same encounter are able to be practiced. Unrehearsed language use is not made available, unless the student is able to come-up with unique discourse that still creates a cohesion and 
coherency to the role-playing situation. Cultural information is available but a close examination of culture is not. Individualized instruction is made available by the many different routes available through the program. The video button on the script screen also allows some user learning preferences and levels of difficulty. Feedback is essentially immediate, but hardly helpful beyond letting the student know the response to the problem was acknowledged. Task variety is good, but the level of interactivity remains at a behaviorist level.

\section{Program Recommendations}

This program provides adequate practice in the speaking, reading, and listening of English found in the useful content presented. I would recommend this program to students wishing to learn and practice the language (including grammar and sociolinguistic information) found essentially in the various situations since no provisions exist for the learning of language strategies for language use outside of the content areas presented. The student using this program should feel comfortable having no computer guidance.

\section{Focus on Grammar, Advanced Level}

(Exceller Software)

\section{Program Description}

This program is one of four in the Focus on Grammar series. Other programs are at the Basic, High-Intermediate, and Intermediate levels (only Intermediate and Advanced are available; the other two are expected to be released sometime in 1997). For use in middle-school, higher education settings, or at home, and designed for use 
with the Focus on Grammar book, this program allows the practice of grammatical structures through a number of reading, writing, and listening activities. The program is composed of 21 units organized by grammatical topic; eight review tests are also included. Each of the units has five sections that allow the user to first "Discover the Grammar," then "Practice" the grammar, then "Read" a passage containing more examples of the grammatical structure, then "Listen" to passage containing the structure, and finally "Write" an essay containing the structure. Each of the five sections has a number of activities using multiple choice, fill-in-the-blank, and dictation formats.

Available to the user are extensive "Grammar Notes" and "Grammar Charts" similar to those found in the Focus on Grammar book. An on-line student workbook provides storage for extensive record information, keeping track both of the overall activity scores and a summary breakdown of those items correct on the first try, those incorrect, those skipped, those not yet attempted, and even those incorrect on the first try and then skipped.

\section{Program Strengths and Weaknesses}

The student workbook is one of the greatest strengths of this grammar-based program. The records, as mentioned above, are an excellent use of the computer's record-keeping capabilities. The user-friendliness of the program is also at a high level. Instructions are essentially adequate, clear, and highly accessible. HELP is always available and program consistency is constant. Navigation through the product is simple and movement to a different part, unit, section, activity, or even a particular activity item is basically only a click away. Not only is navigation easy and fast, but students are also able to know their location at all times; the use of a tool bar provides the location information and allows the user quick access to different parts of the 
program. And students are not locked into a choice/answer once a selection is made. Instead, the user must confirm the selection of the answer by clicking on the $\mathrm{OK}$ button. This feature is available not only for use with answers but also for navigation; the student having so many choices on the scrolled lists can easily click on the wrong line. By having to click on OK students are spared any unnecessary time spent going to the wrong section of the program.

The program does not have any activities that provide the practice of language for use in social settings, nor does it provide activities for the examination of culture. The writing section does ask students to write on such topics as stereotypes, experiences with other cultures, cultural beliefs, and the benefits and disadvantages of categorizing people. Yet there is no assessment of the essay (this can be attributed to current technological shortcomings), nor any chance for students to validate their feelings through the use of any available cultural information. Instead, students are given the topics and left to sort out their feelings alone.

The activities are based on the decontextualized identification and use of grammatical forms. In many of the activities students need only to recognize the structure, and not the meaning of the sentence, to complete the exercise. Exercises are not geared toward communication, although they could be. For example, the structurally based organization of the material could be mixed with a situation-based organization (e.g. at the doctor, at the store, meeting a friend for lunch) and so move away from the program as a grammar workbook in digital format and closer to the expectations stemming from today's teaching approach. Feedback points also to the program's focus on form. The "Your answer is incorrect, please try again" feedback shows the only-one-grammatical-form-is-accepted-for-the-answer theory base used in the development of the program. The predetermined answers always must reflect the 
grammatical structure of the lesson being studied, even though other answers provided would be correct in certain circumstances.

\section{Program Instructional Strengths}

The program does a good job in the creation of a user-friendly learning environment that can affectively help learners as they progress through the program. A variety of tasks provides the user with a number of different activities with which to practice the program's content. Students do not receive any guidance or prompts from the program and are then left to figure out any problems that might arise. The interactivity provided is behavioristically based; all the answers are predetermined and need to be exact, even the spelling errors when typing a response. Individualized instruction exists inasmuch as the student chooses the topic and activity, but there are no provisions made for learning styles, or even levels of difficulty. Communicative language use does not appear to be the focus of the program so there are no chances to use language in realistic and meaningful settings. Students are provided relevant topics regarding the examination of culture, but, as a carrot dangling in front of a horse, the existence of culture-learning only looks real; the actual essence remains out of reach.

\section{Program Recommendations}

I would recommend this program to students desiring behaviorist based drilland-practice exercises for the learning of discrete grammatical structures. The student should be one who can take control of learning because the program provides no guidance (other than providing minimal feedback for activities) through the material. This program is not for those desiring any sociolinguistic language examples, practice, or use. 


\section{CHAPTER V}

\section{DISCUSSION OF THE RESULTS}

This chapter reviews the study and discusses the results of the evaluation. First a summary of results is offered containing a brief overview of the findings. Next, a discussion of the findings presents the results of the survey in the context of the research questions. Finally, conclusions are drawn and implications for ESL and for future software development are made.

\section{Study Overview}

This study was based on the evaluation of ESL CD-ROM software available commercially. Eight software were evaluated using a research tool based on communicative theory widely accepted in today's ESL classrooms. The research questions guiding the evaluation of the software were intended to discover how today's CD-ROM ESL materials account for the non-human instructional component, how in a general way culture is represented and used in the programs, and if the software is providing contextual language use and practice as well as skills that can be transferred to situations outside of the classroom. The overall findings available in a matrix format provide at a glance the conditions of the software in reference to issues of user friendliness, feedback and error treatment, relevance of media used, record keeping, communicative language input and use, and representation of culture. The 
in-depth reviews and critiques provide a closer look at each software in relation to the specific criteria being used in the evaluation tool.

\section{Summary of the Results}

Looking at the results of the evaluation one can see that some non-human instructional components are better provided for than others, and that communicative language and culture issues both have consistently low ratings. Not one single software consistently meets or addresses the criteria supporting the research questions at a level of good or higher for all the points addressed in the evaluation.

Table III shows those components well-provided for in the evaluated materials.

\section{TABLE III}

Components of the Evaluation Receiving Good and/or Excellent Ratings for Half or More of the Software

\begin{tabular}{llll}
\hline User Friendliness & Feedback & Interaction & $\frac{\text { Communicative }}{\text { input \& practice }}$ \\
\hline $\begin{array}{l}\text { Accessible instructions } \\
\text { (4) }\end{array}$ & Feedback is varied (5) & $\begin{array}{l}\text { Levels of difficulty are } \\
\text { available (4) }\end{array}$ & $\begin{array}{l}\text { Topics are suitable for } \\
\text { user (4) }\end{array}$ \\
Help at all time (6) & $\begin{array}{l}\text { Every opportunity for } \\
\text { the correct response (5) }\end{array}$ & $\begin{array}{l}\text { Different questioning } \\
\text { formats used (4) }\end{array}$ & \\
Learner control (4) & $\begin{array}{l}\text { Feedback is well-timed } \\
(8)\end{array}$ & $\begin{array}{l}\text { Questions asked are } \\
\text { relevant to content (8) }\end{array}$ \\
$\begin{array}{l}\text { Consistent interactivity } \\
\text { (6) }\end{array}$ & & \\
\hline
\end{tabular}

Note. Number of software receiving good/excellent scores are shown in parentheses. For complete criteria definitions refer to Appendix B.

Table IV indicates those areas requiring a better representation in today's CALL materials. Six out of the seven instructional design and content components 
evaluated are represented. It is interesting to note that out of the thirteen criteria exploring the user-friendly issue, ten consistently receive low ratings.

TABLE IV

Components of the Evaluation Receiving Weak and/or Adequate Ratings for Half or More of the Software

\begin{tabular}{|c|c|c|c|c|c|}
\hline Friendliness & Feedback & Interaction & $\frac{\text { Record }}{\text { Keeping }}$ & $\frac{\frac{\text { Communica- }}{\text { tive Input \& }}}{\text { Practice }}$ & $\frac{\text { Culture }}{\text { Learning }}$ \\
\hline $\begin{array}{l}\text { Clear and } \\
\text { adequate } \\
\text { instructions } \\
(5)\end{array}$ & $\begin{array}{l}\text { Feedback } \\
\text { constructive } \\
\text { for correct } \\
\text { answers (6) }\end{array}$ & $\begin{array}{l}\text { All reasonable } \\
\text { student } \\
\text { responses } \\
\text { accepted (4) }\end{array}$ & $\begin{array}{l}\text { Program } \\
\text { tracks } \\
\text { movement (4) }\end{array}$ & $\begin{array}{l}\text { Language used } \\
\text { in authentic \& } \\
\text { meaningful } \\
\text { situations ( } 7 \text { ) }\end{array}$ & $\begin{array}{l}\text { Culture is } \\
\text { presented free } \\
\text { of stereotypes, } \\
\text { bias }(6)\end{array}$ \\
\hline $\begin{array}{l}\text { Learner } \\
\text { control of } \\
\text { pace, manner, } \\
\text { direction of } \\
\text { progress(4) }\end{array}$ & $\begin{array}{l}\text { Feedback } \\
\text { constructive } \\
\text { for incorrect } \\
\text { answers (6) }\end{array}$ & $\begin{array}{l}\text { Questions and } \\
\text { prompts are } \\
\text { frequent (4) }\end{array}$ & $\begin{array}{l}\text { Records are } \\
\text { available to } \\
\text { others (4) }\end{array}$ & $\begin{array}{l}\text { Students see } \\
\text { different } \\
\text { contexts of } \\
\text { language use (5) }\end{array}$ & \\
\hline $\begin{array}{l}\text { Can change } \\
\text { most } \\
\text { immediate } \\
\text { input (5) }\end{array}$ & & $\begin{array}{l}\text { Different } \\
\text { questioning } \\
\text { formats used } \\
\text { (4) }\end{array}$ & & $\begin{array}{l}\text { Students are } \\
\text { given } \\
\text { background } \\
\text { information for } \\
\text { context (4) }\end{array}$ & \\
\hline $\begin{array}{l}\text { Choice of } \\
\text { graphics, } \\
\text { instructions } \\
\text { present (5) }\end{array}$ & & $\begin{array}{l}\text { Students can } \\
\text { share in } \\
\text { initiation of } \\
\text { events (6) }\end{array}$ & & $\begin{array}{l}\text { Topics are } \\
\text { suitable for user } \\
\text { (4) }\end{array}$ & \\
\hline \multicolumn{6}{|l|}{$\begin{array}{l}\text { Access to } \\
\text { reports (4) }\end{array}$} \\
\hline \multicolumn{6}{|l|}{$\begin{array}{l}\text { Know } \\
\text { location (5) }\end{array}$} \\
\hline \multicolumn{6}{|l|}{$\begin{array}{l}\text { Clear learning } \\
\text { objectives (5) }\end{array}$} \\
\hline \multicolumn{6}{|l|}{$\begin{array}{l}\text { Easy } \\
\text { navigation (6) }\end{array}$} \\
\hline \multicolumn{6}{|l|}{$\begin{array}{l}\text { Shortcut } \\
\text { features (5) }\end{array}$} \\
\hline $\begin{array}{l}\text { Free of } \\
\text { cultural/sexual } \\
\text { bias (6) }\end{array}$ & & & & & \\
\hline
\end{tabular}

Note. Number of software receiving weak/adequate scores are shown in parentheses. For complete criteria definitions refer to Appendix B. 
Table V suggests that more work needs to be done for the representation of record keeping (four out of the six criteria are totally lacking in more than half of the software) and culture learning (four of its five criteria are totally lacking in half or more of the software). Not shown in the table are results representing the acceptance of reasonable responses; four programs are totally lacking in this area.

\section{TABLE V}

Components of the Evaluation Totally Lacking in Half or More of the Software

\begin{tabular}{lll}
\multicolumn{1}{c}{ Record Keeping } & \multicolumn{1}{c}{ Culture Learning } \\
\hline Test results by student or group (6) & Examination of cultural behavior (7) \\
$\begin{array}{l}\text { Student performance via complete } \\
\text { records }\end{array}$ & $\begin{array}{l}\text { Exercises provide opportunities for } \\
\text { hypotheses (5) }\end{array}$ \\
$\begin{array}{l}\text { Item performance by individual or group } \\
(7)\end{array}$ & Culture integrated in content (5) \\
Tests at beginning and end (5) & Culture treated as supplemental (4) \\
\hline
\end{tabular}

Note. Number of software receiving totally lacking scores are shown in parentheses. For complete criteria definitions refer to Appendix B.

Table VI demonstrates that media is overall well accounted for, especially the use of audio. A wide use of video is obviously lacking. Not shown on the table is the representation of three products that do use video. Two received ratings of good or excellent; the other has an adequate use of video. Surface features, also not represented on tables, show an overall adequacy in their use. Three products show a good or excellent use of surface features. 
TABLE VI

Media Ratings Representing Scores Received

by Half or More of the Software

\begin{tabular}{lllc}
\hline & Low & High & Totally Lacking \\
\hline Aids in Presentation & Photo (4) & Photo (4) & Video (5) \\
& & Audio (8) & \\
& & Text (6) & \\
\hline Useful as Feedback & & & Video (7) \\
& Photo (5) & Audio (5) & \\
\hline
\end{tabular}

Note. Number of software receiving the low/high/totally lacking scores are shown in parentheses. Low refers to scores of 1 (weak) or 2 (adequate); high scores are 3 (good) and 4 (excellent). For complete criteria definitions refer to Appendix B.

Discussion of the Findings

The research questions look for how well software developers are providing for features that make up for the lack of a visible human instructional component, and briefly at the provision of certain content features. As mentioned earlier, not one program consistently provides the instructional features at a level of good or higher. The first area to be discussed refers to those criteria examining the user-friendliness of the program from the perspective of the student. 
User-Friendly Issues

The findings of this project suggest that programs are only minimally concerned with the user-friendly issues that can create a quality learning environment as well as separate a well-thought out program from a hastily made one. Having access to instructions and help is a minimal requirement for any piece of technology and indeed these criteria are well provided for by the programs. The consistent interactivity also shows that the programs' developers are providing users with a regularity that allows the students to become familiar with and proficient at each of the individual routines associated with the respective software; two of the software even provide explicit modes of learning so that students can choose that which best fits their learning style.

What is missing from the user-friendly issues are those features that essentially inform learners and allow learners to make easily reversed choices. With respect to the former, instructions in the programs are weak: suggested paths through the material are not explicitly pointed out, information regarding current or previous location is not good, and learning objectives are not clearly stated. Since the programs are so linear-oriented with minimal uses of hyperlinks, students at least are not likely to get lost. It is interesting that the information available in the accompanying manuals contains far superior instructions and explanations than what is available online; step-by-step explanations with accompanying drawings are frequently found in the manuals, and learning objectives are more clearly stated. In relation to allowing learners easily reversed choices, only one program double checks with learners about their satisfaction with the selection before responding to the input. Otherwise, the programs literally lock students into their selection, either immediately judging their decisions (if an answer was selected) or sending the learner to a destination perhaps chosen by mistake. 
Blum (1995) proposes that the "emerging language of multimedia" (p. 184) will address standards to be maintained as users become more familiar with multimedia products. The standards address the treatment of the user as one who is intelligent, has individual needs, and does not want to be bothered by the operational details of running the program (these details also include knowing location and having a quick access guide/map for movement in the program). For example, Blum says that the use of manuals for instructions is never needed in a well-designed product; any information and explanations that can not be intuitively discerned can be made available by on-line help. This study suggests that developers are not quite yet versed in Blum's "emerging language of multimedia" because many issues mentioned above are overlooked.

\section{Feedback}

All of the feedback found in the programs is immediate and varied. Students not only have immediate acknowledgment that their input is received, but they also are not forced to hear the same feedback over and over (there is not an infinite supply of feedback available; approximately four to six different responses are given, in cycles, and usually accompanied by a graphic symbol and/or sound effects). However, the specific content of the feedback tends toward a more behaviorist application as very little of it is constructive for either correct or incorrect answers. In today's classrooms students rely on immediate teacher and peer feedback to judge the effectiveness of their utterances, similar to what takes place in interactions outside of the classroom. A good teacher will also provide feedback that incorporates language learning strategies. The feedback used in the programs is certainly not communicatively-based. Different variants of "That's not correct, please try again," or, "You are correct" are received by the student; hardly any feedback is used to reinforce why a reply is correct or not. 
Instead, the program seems disinterested and almost impatient to move the student along.

Students also can never really be motivated to get the correct response because of the excessive opportunities given for the correct answer. Cooks and Henstock (1993) suggest that a limited number of tries may provide motivation for students to answer correctly. Otherwise, students will quickly figure out that it doesn't matter to the program what their answer is; the fairly repetitive feedback and its lack of helpfulness can turn a student into a passive presser of buttons until the correct answer is found, and this is what more than half of the programs allow.

The provision of feedback beyond the "correct" and "incorrect" replies is possible, especially within the confines of such controlled linear-tutorial based programs (compared to a more free-ranging application like a simulation). For example, one program does point out why the answer selected is the incorrect choice, thus clarifying the possible misinterpretation of the item being selected. Although most of the activities in the programs rely on one-word responses in fairly decontextualized settings, the program can still supply more creative and helpful feedback. More thought into the type of feedback as well as the anticipation of learner errors would be beneficial, even for drill and practice programs. These answers could still fit into the series of answers repeated at random in the program; instead of the same feedback used across the program, feedback would be based on individual items.

\section{$\underline{\text { Integration of Media }}$}

In any educational classroom, ESL or not, media need to be well-planned and integrated, acting as the best deliverer of the information being presented, even when the media are used for feedback. The use of media in all of the products is well integrated and relevant to what is being presented, except in regard to feedback. 
Feedback is most consistently provided in the form of audio, either through sound effects or oral replies. The much stronger use of audio as feedback compared to the adequate use of pictorial feedback suggests developers might be putting more emphasis on listening skills than on visual association. What contributes most to the low scores received by the photos, used either for the conveyance of core material or feedback, is the images' content quality. In all programs using photos (only one does not), the quality is either consistently good or consistently weak. Vague photos then result in weak scores. Unlike those of one program which are real-life, in color, and highly contextualized photos, other programs use drawings/artwork that do not meet the same level of relevancy. The use of realistic photos can provide a better contextualized referent for the identification of cultural information or for implied language use, not to mention contributing to the visual quality of the program. Good photos and artwork, however, can be rather costly, and finding those that fit the needs of the program takes time and money. Stock photography that provides a virtual encyclopedia of photographic images might be an option, yet even stock photography with its so-called royalty-free privileges is not always royalty-free, and so can become expensive. However, the use of artwork that is not easily associated with the content of the program can become just as costly if it is to create a negative attitude toward the particular program, or even toward the program's maker.

Video use for the programs is low. Only three of the programs use it; two rely on it for the main conveyance of content. Video done well can supply the student with a wealth of contextualized input, both linguistic and sociolinguistic. Although the video used in the two products is scripted and not authentic (authentic material is of course preferable for today's classrooms) it does attempt to provide situations of language use. Overall it appears as if developers are aware of the need not to misuse 
the media. A good balance and careful integration of media are provided, although video representation is low.

\section{$\underline{\text { Interactivity }}$}

To maintain an interest in computer programs, it has been suggested that interactivity be more than "gratuitous mouse clicks to go on to the next screen" (Blum, 1995, p. 94), and that prompts be available for students who might need the reassurance provided by prompts. The use of different question formats and the ability for users to have some say in the events that transpire are further recommended to keep a user's interest. Through the results of the study, it is shown that programs do little to keep the students actively engaged and interested. Instead of acting as a collaborator (as suggested by Renié and Chanier, 1995), the computer is relegated to the role of information provider, a patient machine uninterested in the action taking place, and just waiting for the student to get the correct response so that the program can progress.

The lack of frequent questions or prompts in the programs also does little to keep the student engaged, especially if the student's interest is waning out of boredom, or sheer frustration (remember, the instructions or the reasons for doing the activities are not the best). It seems odd that more prompts are not used, because many of the programs express a desired route for learners to take (this is written in the accompanying user manuals), and because good instructions are not made available. Without prompts, students are not given any personal attention, and thus experience a highly impersonal atmosphere where they are left to their own motivation to find their way through the program.

One program for children does an excellent job with prompts (almost too good; too much help can create passive learners). The prompts engage the learner by making 
sure the user's interaction is progressing smoothly. Students in this program are made to feel as if they are being acknowledged by the "box" they are sitting in front of. Developers need not think that all users share a child's need for reassurance and help. Yet technology can be greatly intimidating to some; the use of prompts adds a degree of friendliness to and interest in the user, thus bringing the experience to a more humanistic level. Of course too many prompts can take away from the student's chance to not feel hurried. However, well-placed and thoughtful prompts can add enjoyment and keep the student interested.

The lack of any prompts or questions shows there is not a "give and take" between the computer and user taking place. Students become passive participants and are not given the chance to share in the initiation of events. Giving students more choices beyond what sections to go to, and involving students more personally in their learning experience can be motivating. The programs evaluated do not provide students with this chance. Instead, students get into the program and follow the regimented path through the one-answer-only responses until the program is finished or the students are done.

\section{$\underline{\text { Use of Single Answers Only }}$}

The use of single-answers-only found essentially throughout all programs (four programs do make slight allowances for other answers, but not consistently) conditions users to behaviorist methods instead of allowing a more free expression and use of language. Even when another answer is just as suitable, the computer maintains a shortsightedness (i.e. not foreseeing other responses as acceptable for the question being asked) by accepting only its predetermined single answer. The interaction between the student and machine thus becomes based on a mechanical exchange of information, hurried through by the "wrong/right" feedback, where only the 
computer's needs are met. If one looks at the context of the one-answer-only provisions, the types of activities, one quickly sees why the one-answer-only rule applies.

\section{One-word Level Exercises}

Almost all of the programs supply a variety of questioning formats with some using the multiple choice-, true/false-, fill-in-the-blank-, matching-, and unscrambleformats more creatively than others. However, the practice taking place does not go much beyond one-word answers. If the activities are not comprehension-based, they are mainly based on the teaching of discrete linguistic segments, either grammaticallyor lexically-oriented. Therefore, for the type of programs and the highly structured activities provided, one-answer-only responses make sense. If activities had been geared more toward communication, one-answer-only responses at a word-level would not have been acceptable.

\section{Levels of Difficulty Provided}

The use of levels of difficulty is meant to meet the individual needs of the intended users. Half of the programs do provide levels of difficulty. Yet in developers' attempts to provide for a large audience, two are over-zealous. Their programs are made for students age eight to adult; another product is for ages eight to sixty. It is hard to believe that what can be appealing to an eight-year-old has the same appeal to a sixty-year-old. Truett and Gillespie state that "using the software across all levels [is] akin to offering exercises from the same workbook to students from kindergarten to senior high - far above the level of some, insultingly below the capabilities of others" $(1984$, p. 36). The majority of software does provide 
reasonable age expectations for the product-user; only the two mentioned above do not.

\section{$\underline{\text { Record Keeping }}$}

With the strength of the computer as a record keeper, made even easier by the student input that is available as quantitative data, it seems a waste to not use the record-keeping capabilities. Yet only one product uses the record-keeping abilities well. With record keeping, not only can teachers access the data, but developers could use it to make products better, and students are provided with a means to rate their performance. If the program does not provide students with problem-solving or realworld tasks with which to gauge their linguistic effectiveness, at least records can provide the latest level attained or the latest score reached and thus provide some sort of motivation. Granted the use of records means that more time needs to be spent in the programming of the software, yet in the long run the advantages can work for instructors, students, and developers.

Until now the discussion has been focused on how CD-ROM ESL materials account for the non-human instructional component in relation to a number of issues. Also addressed in this study are two areas representing the general content of the material: the representation of culture, and contextual language input and practice for use outside of the classroom. Both areas received fairly low scores; the provision of culture learning is almost totally lacking in all software evaluated.

\section{Communicative Language Input}

The provision of language in meaningful and realistic samples that can be used in different contexts can provide students with the opportunity to experience and 
practice language in a non-intimidating environment. While many of the programs are advertised as providing students with the opportunity to practice language for use in a number of situations, few provide the various situations and the meaningful language for students to use. Instead, students act as outside observers, using language only to speak of others, not of themselves. And, students are never shown how or why language use can change across contexts and participants. Only two programs provide students with the opportunity to experience and practice language at a communicative level (i.e. above one-word responses). Although the practice provided is based on routinized exchanges, students can try out language in fairly authentic situations that can prepare students for real-world interactions.

\section{$\underline{\text { Technology and Oral Practice }}$}

Although how language practice is provided is not addressed in the study, seven out of the eight software have a record/playback feature available, and three use speech recognition. The students are then provided an opportunity for oral practice, but the practice is not well-integrated with the material (the use of record/playback is not as fully integrated a component as the use of speech recognition). Only two programs do a good job of integrating the oral-practice feature into the program. As much as the programs provide the students the opportunity to speak by the inclusion of these features, if the features are not integrated or understood by the user, the usefulness becomes rather limited. In the accompanying literature suggestions for how better to use the features are available, but no instruction is given on-line.

\section{Culture Learning}

The lack of activities or information addressing culture and culture-learning skills in many of the programs shows a disinterest in, or unawareness of, the need for 
its provision. Two of the programs actually have "culture" activities or information explicitly available, instead of an indirect presentation through content (often seen as biases or stereotypes). The activities and information presented by the two programs provide a good place to start - yet it is only at a surface level. There is no look at how cultural behavior might cause misunderstandings. Exercises (some based on the use of definitive cultural facts), provide the opportunity to accept, reject, or modify existing hypotheses only by the nature of the culturally bound content. There are no actual activities where students can explore their own feelings and attitudes. Instead, students are treated as passive receptacles of the program's often biased viewpoints, and can do nothing about it.

\section{Conclusions and Implications}

The present study had as its motivation the desire to see how present-day CALL CD-ROM material accounts for specific instructional components when a human instructor is not present, as well as providing for content issues relevant to today's classrooms. Using a communicative theory base from which to judge and rate the programs, an evaluation was conducted. The results of the study suggest that although most of the instructional components are acknowledged and addressed, it is at a minimal level; the components do not go far beyond the behaviorist drill and practice programs of the past. Today's programs are still characterized by one-answer only responses, decontextualized word-level exercises, passive student involvement, and "wrong/right" feedback. In terms of the inclusion of culture learning and contextual communicative language input and practice, the findings suggest much work needs to be done. 
In general, almost every software has its own particular strengths and weaknesses in reference to the research questions and the theory guiding the study. If all software strengths could be combined into one product, the goal of making software more grounded in today's teaching theory could be almost realized. As it stands, this is not the case. Developers appear to recognize some, but not all of, the needs of today's students. Developers might also be misinformed about today's ESL environment, or they may just not be able or willing to take the time to provide a product soundly based on the current teaching approach.

Many of the findings suggest that there has been haste in the development of products; in the need to get a product to market, the instructional design and content features looked for in this study appear to be left behind. Because of the fairly competitive nature of the commercial software industry, product design, from beginning plans to creation, is always in a race against the next company who is trying to do almost the same thing, but faster. The first generalization to make about today's commercially available CALL software is that developers may not be putting enough effort into the product to satisfy the needs of today's ESL student.

Poor instructions, poor identification of learning objectives, and poor representation of culture learning and communicative activities are representative of many products. Yet good instructions and the provision of other features lacking online are available in the accompanying literature. It is easier to write the information than to creatively contain it within the program. Developing and programming costs, and time constraints most likely hinder the inclusion of the features on-line even if technology makes them possible to include in the program itself. These feature's inclusion on-line can enhance a product and in the long run may promote a good product reputation and, by extension, better consumer allegiance. 
Developers are also barely using technology's potential. Hypertechnology is not used; the paths through the material are linear and fast - there are no divergences for students and no chances to satisfy individual needs of learning. Feedback, although varied, tends to be repetitive and applied globally to a program. By spending more time and providing feedback to individual exercises, feedback can become more constructive. The use of single-answers not only reinforces the ideology of the producer, but also makes error recognition simple and fast; not much time needs to be spent anticipating and programming to recognize other forms of acceptable responses. The lack of record keeping is also an easy way for developers to cut production time; its lack of inclusion in the products signals developers' disinterest in the user's need for evaluation or data that can be used to make a better product. Not using prompts to keep user interest also shows how technology is not being used. The use of repetitive interaction through consistent interactivity, good for the infancy stages of CALL as students become acquainted with the "routines" of computer use, also shows a fast and easy way for developers to cut production time and costs. The controlled practice activities are repetitive, with no allotment of unpredictable outcomes that can be motivating. Producers do, however, exhibit the knowledge of wisely incorporating media into the products. In the programs evaluated, hardly any media are misused or overused. Except for the vagueness of some of the product photos, most did a good job with the presentation of media.

Much of what developers are offering would hardly stand up in today's ESL classroom if it were not delivered through a computer. This is interesting because many of the evaluated products, as stated in their product literature, have educators and ESL professionals involved with the creation of the product. The products, however, still do not seem to reflect the current communicative teaching approach. In the materials there are no exchanges of information and few chances for students to 
share in the initiation of events, let alone receive communicative input and practice. Error feedback does not contribute to language learning strategies; one-word-only answers do not meet the discourse level used in classrooms. This leads to generalization number two: developers are not using a communicative approach to language instruction and learning.

Hutchinson and Waters infer that it is "now almost an insult to infer that someone's materials...are uncommunicative" (1987, p. 23). Drawing from the theory base discussed in Chapter II, the programs evaluated are quite far from being anything but uncommunicative. Instead, many of the programs seem to follow more of a comprehension-based approach. In this approach receptive skills (i.e. listening) are given priority over productive skills (i.e. speaking). Many of the software are advertised as having a natural approach to learning. The natural approach as developed by Krashen and Terrell (1983) is itself comprehension-based. This approach is based on the provision of input, with no language production until the student feels ready to do so. When students area ready to speak, they start first with one-word responses and ultimately build up to discourse segments. This approach is thought to be similar to how a child learns its first language. The natural approach reflects the communicative approach in that neither focuses primarily on grammatical accuracy; instead, self-expression and the negotiation of meaning are stressed. Yet in the programs evaluated even these features are not provided for; students are almost never given the opportunity to express themselves freely or to negotiate meaning.

The third and final generalization of the evaluation is based on consumers and developers' perceptions of consumers' needs. The nature of material for consumers is usually based on their perceived needs. It could be that developers are misinformed about today's ESL needs, although one would think ESL professionals on the team would remedy that. However, with the programs evaluated, it appears that producers 
are gearing their products toward uninformed users and instructors who are not fully aware of the capabilities of the computer and those who don't step in to demand a better product. Perhaps the programs adequately reflect the state of what ESL educators know of and expect from multimedia products. Too many educators may still be caught up in the glamour of multimedia and not have the knowledge to make informed decisions regarding its current state. As a result, through the use of advertising "buzzwords" that represent the qualities of a good program, inexperienced users and instructors fall prey to the proclaimed worth of the program. In time, as both students and teachers become more knowledgeable of and used to more engaging and relevant computer applications, many of the programs evaluated in the study will lose their competitive edge.

In the end it looks as if a tool meant for a communicative-based group of software was applied to a group of non-communicative material. The guiding philosophies behind each software's instructional design and inclusion of content hardly meet up with the philosophy that guided this study. The results show that much work needs to be done in order to take commercial software to the next level (i.e. beyond drill-and-practice multimedia). A closer relationship between the consumer and developer might be beneficial in that the latter could be informed of likes and dislikes of the product. Only one out of the eight programs asks for user feedback regarding the use of the products; all others express a self-interest by asking users to call them for more information. Another suggestion is to make the university-specific programs available to a wider audience. The final suggestion will most likely come in time: it is the need for an educated and informed market who can then demand a better product within the means of technology. 


\section{REFERENCES}

Al-Arishi, A. Y. (1994). An integrated approach to the use of technology in communicative language teaching. Language Quarterly, 32, (3-4), 175-191.

Ambron, S., \& Hooper, K. (1990). In S. Ambron \& K. Hooper (Eds.), Learning with interactive multimedia, developing and using multimedia tools in education. Redmond: Microsoft Press.

Bedford, A. M. Z. (1991). Methodology for CALL - beyond language teaching paradigms. In W. Brierley \& I. R. Kemble (Eds.), Computers as a tool in language teaching (pp. 161-173). West Sussex: Ellis Horwood Limited.

Belch, G. E., \& Belch, M. A. (1995). Introduction to advertising and promotion, an integrated marketing communications perspective. Chicago: Richard D. Irwin, Inc.

Blum, B. (1995). Interactive media - essentials for success. Emeryville: ZiffDavis Press.

Borras, I. (1993). "Practicing spoken French" (PSF): Multimedia courseware for improving oral communicative skills. In F. L. Borchardt \& E. M. T. Johnson (Eds.), Proceedings of the Computer Assisted Learning and Instruction Consortium (CALICO) 1993 Annual Symposium on "Assessment" (pp. 16-20). Durham, NC: Duke University Press. 
Brierley, W. (1991). Natural language processing. In W. Brierley \& I. R. Kemble (Eds.), Computers as a tool in language teaching (pp. 71-85). West Sussex: Ellis Horwood Limited.

Brinton, D. M. (1991). The use of media in language teaching. In M. CelceMurcia (Ed.), Teaching English as a second or foreign language (pp. 454-471). Boston: Heinle \& Heinle Publishers.

Brock, P. A. (1994). Educational technology in the classroom. Englewood Cliffs, N.J.: Educational Technology Publications, Inc.

Brown, E. (1997, February 10). DVD: The hype and the hope. New Media: The Magazine for Creators of the Digital Future, 44-51.

Brown, H. D. (1993). TESOL at twenty-five: What are the issues? In S. Silberstein (Ed.), State of the art TESOL essays, celebrating 25 years of the discipline (pp. 16-34). Bloomington, IL: Pantagraph Printing.

Brown, H. D. (1994). Principles of language learning and teaching. Englewood Cliffs, NJ: Prentice Hall Regents.

Brown, J. D. (1995). The elements of language curriculum - a systematic approach to program development. Boston: Heinle \& Heinle Publishers. 
Brown, K. (1995, October). Intercultural competence from a research perspective. Lecture for Culture Learning in the Language Classroom, Portland State University, Portland, OR.

Byram, M. (1988). Foreign language education and cultural studies. Language, Culture, and Curriculum, 1, (1), 15-31.

Carrick, B. (1988). TESL software description: including an annotated catalog of commercially available ESL software. Unpublished master's thesis, Portland State University, Portland, Oregon.

Celce-Murcia, M. (1991). Language teaching approaches: An overview. In M. Celce-Murcia (Ed.), Teaching English as a second or foreign language (pp. 3-11). Boston: Heinle \& Heinle Publishers.

Celce-Murcia, M. (1993). Grammar pedagogy in second and foreign language teaching. In S. Silberstein (Ed.), State of the art TESOL essays, celebrating 25 years of the discipline (pp. 288-309). Bloomington, Illinois: Pantagraph Printing.

Chaika, E. (1989). Language - the social mirror. Cambridge: Newbury House Publishers.

Chan, C., \& Liu, L-C. (1992). Yu-gong yi shan: Exploring some possibilities of designing tomorrow's foreign language textbook. CALICO Journal, 9, (4), 49-59. 
Cheung, A., \& Harrison, C. (1992). Microcomputer adventure games and second language acquisition: A study of Hong Kong tertiary students. In M.

Pennington \& V. Stevens (Eds.), Computers in applied linguistics: An international perspective (pp. 66-76). Bristol, PA.: Multilingual Matters, Ltd.

Cohen, A. D. (1996). Developing the ability to perform speech acts. Studies in Second Language Acquisition, 18, (2), 253-267.

Coleman, J. (1991). Interactive Multimedia. In W. Brierley \& I. R. Kemble (Eds.), Computers as a tool in language teaching (pp. 87-111). West Sussex: Ellis Horwood Limited.

Conrad, K. B. (1996). CALL - non-English L2 instruction. Annual Review of Applied Linguistics, 16, 158-181.

Cooks, M. \& Henstock, P. (1993). Improved efficiency in computer-based language instruction. In F. L. Borchardt \& E. M. T. Johnson (Eds.), Proceedings of the Computer Assisted Learning and Instruction Consortium (CALICO) 1993 Annunal Symposium on "Assessment" (pp. 39-43). Durham, NC: Duke University Press.

Cooper, P. (1993). Communication and gender in the classroom. In L. P. Arliss \& D. J. Borisoff (Eds.), Women \& men communicating: challenges and changes (122-141). Fortworth: Harcourt Brace \& Company. 
Crookes, G., \& Chaudron, C. (1991). Guidelines for classroom language teaching. In M. Celce-Murcia (Ed.), Teaching English as a second or foreign language (pp. 46-67). Boston: Heinle \& Heinle Publishers.

Damen, L. (1987). Culture learning: the fifth dimension in the language classroom. Reading, MA: Addison-Wesley.

Davey, D., Jones, K. G., \& Fox, J. (1995). Multimedia for language learning: Some course design issues. Computer Assisted Language Learning, 18, (1), 31-44.

Dubin, F., \& Olshtain, E. (1986). Course design - developing programs and materials for language learning. Cambridge: Cambridge University Press.

Eisele, J. E., \& Eisele, M. E. (1990). Educational technology - a planning and resource guide supporting curriculum. New York: Garland Publishing, Inc.

Flagg, B. N. (1990). Formative evaluation for educational technologies. Hillsdale, NJ: Lawrence Erlbaum Associates.

Ganszauge, M., Hult, J., Sajavaara, K., \& Konttinen, R. (1994). The computer in the English language classroom. Scandinavian Journal of Educational Research, 38, (2), 159-174.

Guglielmino, L. M. (1991). Adult ESL instruction: A sourcebook. Glenview, IL: Scott, Foresman and Company. 
Hanson, B. (1996, October). Distance learning: a new teaching paradigm. Presentation at the Oregon Multimedia Education Conference, Canby, OR.

Harland, M. (1991). Hypermedia. In W. Brierley \& I. R. Kemble (Eds.), Computers as a tool in language teaching (pp. 145-160). West Sussex: Ellis Horwood Limited.

Higgins, J., \& Johns, T. (1984). Computers in language learning. London: Collins ELT.

Hooper, K. (1990). Hyper card: A key to educational computing. In S. Ambron \& K. Hooper (Eds.), Learning with interactive multimedia, developing and using multimedia tools in education (pp. 5-26). Redmond: Microsoft Press.

Hoopes, D. S. (1979). Intercultural communication concepts and the psychology of intercultural experience. In M. D. Pusch (Ed.), Multicultural education: A cross-cultural training approach (pp. 10-38). Chicago: Intercultural Network, Inc.

Hubbard, P. (1992). A methodological framework for CALL courseware development. In M. Pennington and V. Stevens (Eds.), Computers in applied linguistics: An international perspective (pp. 39-65). Bristol, PA: Multilingual Matters, Ltd.

Hutchinson, T,. \& Waters, A. (1987). English for specific purposes: A learning-centered approach. Cambridge: Cambridge University Press. 
Hymes, D. (1974). Foundations in sociolinguistics - an ethnographic approach. University of Pennsylvania Press: Philadelphia.

Ito, Y. (1996). Communication between high school and college Japanese language education: Implications from a survey on the use of video materials in the United States. Foreign Language Annals, 29, (3), 463-479.

Kenning, M-M., \& Kenning, M. J. (1990). Computers \& language learning, current theory \& practice. West Sussex: Ellis Harwood Limited.

Kramsch, C. (1993). Context and culture in language teaching. Oxford: Oxford University Press.

Krashen, S. D. \& Terrell, T. D. (1983). The natural approach: Language acquisition in the classroom. San Francisco: Alemany Press.

Larsen-Freeman, D. (1991). Teaching grammar. In Marianne Celce-Murcia (Ed.), Teaching English as a second or foreign language (pp. 279-296). Boston: Heinle \& Heinle Publishers.

Larsen-Freeman, D., \& Long, M. H. (1991). An introduction to second language acquisition research. London: Longman Press Group U.K.

Layne, P. P., \& Lepeintre, S. (1996). Distance instruction. Annual Review of Applied Linguistics, 16, 226-239. 
Lee, S-C. (1996). Perceptual considerations in icon design for instructional communication. Educational Technology, 36, (2), 58-60.

Lian, A. (1992). Intelligence in computer-aided language learning. In M. C. Pennington \& V. Stevens (Eds.), Computers in applied linguistics: an intercultural perspective (pp. 66-76). Bristol, PA: Multilingual Matters, Ltd.

Liou, H-C. (1993). Effective human-computer interaction in IVD/CALL design and evaluation: Human factors. In F. L. Borchardt \& E. M. T. Johnson (Eds.), Proceedings of the Computer Assisted Learning and Instruction Consortium (CALICO) 1993 Annunal Symposium on "Assessment" (pp. 16-20). Durham, NC: Duke University Press.

Liou, H-C. (1994). Practical considerations for multimedia courseware development: An EFL IVD experience. CALICO Journal, 11, (3), 47-74.

Littlewood, W. (1981). Communicative language teaching - an introduction. Cambridge: Cambridge University Press.

Luskin, B. J. (1996). Toward an understanding of media psychology. Technological Horizons in Education (T.H.E.) Journal , 23, (7), 82-84.

Lyman-Hagar, M. (1995). Multitasking, multilevel, multimedia software for intermediate-level French language instruction: Ça continue.... Foreign Language Annals, 28, (2), 179-192. 
Meskill, C., \& Swan, K. (1993). Assessing CALL software: A computerized database approach. In F. L. Borchardt \& E. M. T. Johnson (Eds.), Proceedings of the Computer Assisted Learning and Instruction Consortium (CALICO) 1993 Annunal Symposium on "Assessment" (pp. 104-106). Durham, NC: Duke University Press.

Mohan, B. (1992). Models of the role of the computer in second language development. In M. Pennington \& V. Stevens (Eds.), Computers in applied linguistics: An international perspective (pp. 110-125). Bristol, PA: Multilingual Matters, Ltd.

Nanny, M. (1990). Interactive images for education. In S. Ambron \& K. Hooper (Eds.), Learning with interactive multimedia, developing and using multimedia tools in education (pp. 100-110). Redmond: Microsoft Press.

Nelson, G. (1995). Considering culture: Guidelines for ESL/EFL textbook writers. In P. Byrd (Ed.), Material writer's guide (pp. 23-44). Boston: Heinle \& Heinle Publishers.

Nunan, D. (1992). Research methods in language learning. Cambridge: Cambridge University Press.

Nunan, D. (1993). Communicative tasks and the language curriculum. In S. Silberstein (Ed.), State of the art TESOL essays, celebrating 25 years of the discipline (pp. 52-68). Bloomington, Illinois: Pantagraph Printing. 
Olshtain, E., \& Cohen, A. (1991). Teaching speech act behavior to nonnative speakers. In M. Celce-Murcia (Ed.), Teaching English as a second or foreign language (pp. 154 - 165). Boston: Heinle \& Heinle Publishers.

Oxford, R. (1990). Language learning strategies. New York: Newbury House.

Pacino, M. A. \& Pacino, J. L. (1996). Multimedia and cultural diversity. Technological Horizons in Education (T.H.E.) Journal, 23, (6), 70-71.

Peck, S. (1991). Recognizing and meeting the needs of ESL students. In M. Celce-Murcia (Ed.), Teaching English as a second or foreign language (pp. 363-372). Boston: Heinle \& Heinle Publishers.

Pica, T., \& Doughty, C. (1985). Input and interaction in the communicative language classroom: A comparison of teacher-fronted and group activities. In S. M. Gass \& C. G. Madden (Eds.), Input in second language acquisition (pp. 115-132). Rowley, MA: Newbury House Publishers, Inc.

Puragson, K. B. (1991). Planning lessons and units. In M. Celce-Murcia (Ed.), Teaching English as a second or foreign language (pp. 419-431). Boston: Heinle \& Heinle Publishers.

Renié, D., \& Chanier, T. (1995). Collaboration and computer-assisted acquisition of a second language. Computer assisted language learning 8, (1), 3-29. 
Rivers, W. M. (1976). Talking off the top of their heads. In W. M. Rivers (Ed.), Speaking in many tongues: Essays in foreign language teaching (pp. 21-35). Rowley, MA: Newbury House Publishers, Inc.

Rühlmann, F. (1995). Towards replacement of the teaching process: the emulation of the teaching process with CAL and its implications for the design of a multimedia CAL tutorial. Computer Assisted Language Learning, 8, (1), 45-61.

Savignon, S. J. (1983). Communicative competence: Theory and classroom practice. Reading, MA: Addison-Wesley Publishing Company.

Savignon, S. (1993). Communicative language teaching: State of the art. In S. Silberstein (Ed.), State of the art TESOL essays, celebrating 25 years of the discipline (pp. 35-51). Bloomington, Illinois: Pantagraph Printing.

Saville-Troike, M. (1985). Cultural input in second language learning. In S. M, Gass \& C. G. Madden (Eds.), Input in second language acquisition (pp. 51-58). Rowley, MA: Newbury House Publishers, Inc.

Scarcella, R. (1990). Teaching language minority students in the multicultural classroom. Englewood Cliffs, NJ: Prentice Hall Regents.

Schreck, R. \& Schreck, J. (1991). Computer-assisted language learning. In M. Celce-Murcia (Ed.), Teaching English as a second or foreign language (pp. 472486). Boston: Heinle \& Heinle Publishers. 
Skierso, A. (1991). Textbook selection and evaluation. In M. Celce-Murcia (Ed.), Teaching English as a second or foreign language (pp. 432-453). Boston: Heinle \& Heinle Publishers.

Stevens, V. (1993). Humanism and CALL: A coming of age. In M.C. Pennington \& V. Stevens (Eds.), Computers in applied linguistics: An international perspective (pp. 11-37). Bristol, PA: Multilingual Matters Ltd.

Sussex, R. (1996). The production and consumption of text. Annual Review of Applied Linguistics 16, 46-67.

Swain, M. (1985). Communicative competence: Some roles of comprehensible input and comprehensible output in its development. In S. M. Gass \& C. G. Madden (Eds.), Input in second language acquisition (pp. 235-253). Rowley, MA: Newbury House Publishers, Inc.

Taylor, E. W. (1994). Intercultural competency: A transformative learning process. Adult Education Quarterly, 44, (3), 154-174.

Thomas, J. (1986). Adapting dbase II: The use of database management systems in English language. In G. Leech \& C.N. Candlin (Eds.), Computers in English language teaching and research (pp. 112-131). Essex: Longman Group Limited.

Tomalin, B. , \& Stempleski, S. (1993). Cultural awareness. Oxford: Oxford University Press. 
Truett, C., \& Gillespie, L. (1984). Choosing educational software - a buyer's guide. Littleton, $\mathrm{CO}$ : Libraries Unlimited, Inc.

Tuman, M. (1996) Literacy on-line. Annual Review of Applied Linguistics 16, 26-45.

Windeatt, S. (1986). Observing CALL in action. In G. Leech \& C.N. Candlin (Eds.), Computers in English language teaching and research (pp. 79-97). Essex: Longman Group Limited.

Yalden, J. (1983). The communicative syllabus - evolution, design, and implementation. Oxford: Pergamon Press.

Internet Addresses:

http: |lwww.mail.dyned.com -- DyNed International http: ॥www.trstone.com -- Rosetta Stone http:॥www.agoralang.com -- Agora Marketplace http:\|www.bayware.com -- Bayware Software 


\section{APPENDIX A}

Software Companies Represented in the Study

The Learning Company

6493 Kaiser Drive

Freemont, CA 94555

Intechnica International

2713 Villa Prom, Suite 7

Oklahoma City, OK 73107

DyNed International

989 E. Hillsdale Blvd, \# 130

Foster City, CA 94404

Exceller Software Corporation

2 Graham Road West

Ithaca, NY 14850
CALI Incorporated

734 E. Utah Valley Drive

American Fork, UT 84003

Fairfield Language Technologies

122 South Main Street

Harrisonburg, VA 22801

Syracuse Language Systems

5790 Widewaters Parkway

Syracuse, NY 13214 


\section{APPENDIX B}

Evaluation Tool and Sources

\section{Aspects of Student Use}

\section{A. User friendliness and learning environment}

1. Instructions

a. Are adequate and clear $(5,6)$

b. Are accessible $(7,5,11)$

2. HELP feature

a. Is available in any form, at any time $(5,6)$

3. Learner control

a. Learners are given adequate control of pace, manner, and direction of progress $(5,6,10,11)$

b. Learners can change their most immediate input $(5,6,9)$

c. Learners can choose not to have graphics, instructions, sound, etc. present $(5,10,11)$ 
d. Learners have access to meaningful and readable progress reports (6)

e. Students are able to know their location

$\begin{array}{lllll}0 & 1 & 2 & 3 & 4\end{array}$ in the program at any time (10)

4. Program consistency and learner's expectations

a. Interactivity is consistently similar $(5,6,10)$

b. Learning objectives are clearly stated or easily identified (6)

5. Movement within program (mechanical considerations)

$\begin{array}{lllll}0 & 1 & 2 & 3 & 4\end{array}$

a. Students can navigate freely and easily $(5,10,11)$

b. Special shortcut features exist for seasoned users (9)

6. Program is free of bias (sexually, culturally) 


\section{Aspects of Instructional Design}

\section{B. Feedback and error treatment}

1. Feedback is varied $(5,11)$

2. Feedback is constructive for correct answers $(5,10,11,6,9)$

3. Feedback is constructive for incorrect answers $(5,10,11,6,9)$

4. Program allows every opportunity for the correct response (6)

5. Feedback is well-timed $(10,6,11)$ 


\section{Relevance of media's input role/ aesthetic surface features' relevancy}

\section{Media (photo, video, text, audio)}

1. Media aids in the presentation of material/activity $(3,5,7)$

role of photo

$\begin{array}{lllll}0 & 1 & 2 & 3 & 4\end{array}$

role of video

$\begin{array}{lllll}0 & 1 & 2 & 3 & 4\end{array}$

role of audio

$\begin{array}{lllll}0 & 1 & 2 & 3 & 4\end{array}$

role of text

$\begin{array}{lllll}0 & 1 & 2 & 3 & 4\end{array}$

2. Media is useful as feedback $(3,11,5,7)$

role of photo

role of video

$\begin{array}{lllll}0 & 1 & 2 & 3 & 4\end{array}$

role of audio

role of text

\section{Surface features}

1. Colors, type faces, borders, underlining, etc. are understandable and helpful in the identification of key learning points $(5,11)$ 


\section{Interaction of student and software}

1. Different levels of difficulty are available (11)

2. All reasonable student responses are accepted $(5,6)$

3. Questions and prompts are frequent $(5,6)$

4. Different questioning formats are used (6)

5. Questions asked are relevant to content $(5,6)$

6. Students are able to share in the initiation of events (9) 


\section{E. Record keeping}

1. The program adequately tracks movement (5)

2. Records of the learner's activities and performance are available to specific people $(6,10)$

3. Besides attendance, program generates other reports

a. Test results by student or group (6)

b. Student performance via complete records, activities, tests (6)

$\begin{array}{lllll}0 & 1 & 2 & 3 & 4\end{array}$

c. Item performance by individuals or group (6)

4. Tests are included at the beginning and end for diagnostic reasons (6)

$\begin{array}{lllll}0 & 1 & 2 & 3 & 4\end{array}$

SCORING: 0 = totally lacking; 1 = weak; 3 = good; 4 = excellent 


\section{F. Contextual communicative language input and use for preparation of the world outside}

1. Linguistic items are used in authentic situations and in meaningful situations that portray accurate, real-world interactions $(7,8,12)$

2. Students are able to see how different context/participants require different/similar language use $(7,8)$

3. Students are given enough background information (i.e. cultural information, setting, etc.) so that they can understand the context of language use (8)

4. Subject matter covers an array of suitable topics for the user (7) 


\section{G. Representation of culture}

1. Exercises allow students to recognize cultural behavior as a possible catalyst of misunderstanding (1)

2. Exercises provide opportunity for the acceptance, rejection, or modification of culture hypotheses (1)

3. Culture is presented free of bias, prejudice and stereotypes (7)

$\begin{array}{lllll}0 & 1 & 2 & 3 & 4\end{array}$

4. Culture is presented through integration of texts, dialogues and exercises (7)

5. Culture is treated as supplemental or optional (7) 


\section{Summary Evaluation}

1. What are the noteworthy strengths? Weaknesses? (6)

2. Would you recommend the software? Why? Why not? (6)

3. Does the program take advantage of the computer's instructional strengths? (11)

Sources Consulted for the Creation of the Evaluation Tool

1* Nelson, G., 1995.

2 Brown, J. D., 1995

3 Brinton, D. M., 1991

4 Coleman, J., 1991

5 Schreck, R., \& Schreck, J., 1991

6 Eisele, J. E., \& Eisele, M. E., 1990
7 Skierso, A., 1991

8 Dubin, F., \& Olshtain, E., 1986

9 Liou, H-C., 1993

10 Flagg, B. N., 1990

11 Truett, C., \& Gillespie, L., 1984

12 Renié, D., \& Chanier, T., 1995

* The numbers are used for source identification for each of the evaluation criteria 


\section{APPENDIX C}

\section{Sample Pages from a "Naive User" Log}

I've just turned the computer on, and I opened the program. I see some animation, a map, with the company's logo, and I hear sound. It's too loud. Where do I turn it down? Is there a button on the computer? Or am I stuck with this being too loud? Do I have to find it somewhere in the program? Oh- here's my friend. He showed me the control on the computer (what would I have done if my friend wasn't here?). O.K. A screen has appeared, asking me to Identify myself. It tells me (in writing) to type at the blinking line the number of students working together. Well, it's just me. I typed "1" so now what? Nothing has happened although there now is a one in the box, and the flashing line is still there. At the bottom of the screen it tells me to use the keyboard for this screen. O.K, I did that, but what do I do now? Nothing has happened. If I use the arrows nothing happens. If I try "Escape", nothing happens either. If I randomly pound away at any combination of keys, no response. The computer just beeps at me. What about the ENTER-key? My friend told me something about that big long key. I can't remember what, though. I press it. Yes, something happens, the screen has changed, and it's now telling me I'm User 1, and it's asking me for my last name. I type it in. Oops, I spelled my name wrong - and it's taking me forever because I haven't quite learned where all the keys are (especially since I'm only used to a Cyrillic keyboard, or Arabic, or...). But I accidentally hit the ENTER-key, so I'm worried because already I've made a mistake. But isn't it supposed to know I made a mistake? Aren't computers supposed to be smart? Well, anyway, it has asked me to enter my first name now. Enter (it never told me I had to press ENTER - I'm just doing it now because it worked the first time). Now they want 
me to type my ID \#. What was that number my teacher told us to use? I wrote it somewhere. Here it is - But one is my ID number, and the other one is the password. I guess they want the number? I'll try it: 222 . Enter. And then my password. Enter. Now it asks me if the information is correct. I need to type $\mathrm{Y}$ or $\mathrm{N}$, they tell (write) me. I type $\mathrm{N}$. The screen changes, and asks me again for my last name, and then first name (but it was only my last name incorrect! Dumb computer!). But I type it again, I don't know why...ENTER. Now I am at a screen that says MAIN ACTIVITY MENU at the top. Hmm. It looks as if by moving the cursor around on the screen that different boxes change color. There is a box with CONVERSATIONS on it; CONVERSATIONS WITH CHOICES; CONVERSATIONS BY GRAMMAR TOPIC; PRACTICE ACTIVITIES; SPEAKING ACTIVITIES; LISTENING ACTIVITIES; SKILL CHECK; YOUR PERFORMANCE STATUS. These buttons light up as my cursor passes over them. There are eight choices, and at the bottom there are other buttons, labeled A-ABOUT; X- QUIT All, E-EXIT, H-HELP. Where do I start? I should have paid more attention to the teacher when she was telling us about this stuff. I want to try SPEAKING ACTIVITIES. I remember she said Point and Click. I'll get the arrow on the box, and it lights up, now Click. Good! I'm at a new screen. It's asking me which speaking activity (this is written). Well, how many are there? It shows PRONUNCIATION, and then another box that has BACK TO MENU. I'll try PRONUNCIATION. Click. Good-something happened. There is now a screen telling me to "Select one below". There are arrows pointing, and the three boxes light up when my cursor goes over them. O.K. Here goes. Click. Wow! There is a screen now with a lot of stuff on it. What do "fricatives", "affricates" mean? There's a noticeable area on the left of the screen, a much different color than the rest. It stands out. It has some words there. From what I understand, they seem to be directions. I'm sick of directions! I want the thing to just run! Doesn't a computer 
know what to do? I'm getting the feeling that I'm going to be doing all the work here...Well, I see what I am supposed to do- if I click on a button, a woman appears in the place the directions were, a real woman, and she makes a sound. It is the sound of the button I clicked on. And other words appear under her, words that start with the letter of the button I clicked on. The key I clicked on remains lit up, and I can hear all the words that appear, or just select one or two. How do I do another sound? O.K. I just click on the little boxes with the letters. But I don't recognize what $[\mathrm{XX}]$ is. It looks like an $\mathrm{N}$ and $\mathrm{G}$ together. It is on the same box that the ng are on. I wonder what that is...What does PROFILE VIEW, or X-RAY VIEW mean? Click. Oh. XRAY view show a cartoon like picture of the mouth. If I click on a sound box (with the letters on them) the sound is made three times, and there is some movement in the cartoon mouth. O.K. I'm bored. I'm going to try those boxes at the way bottom of the screen. HELP. Click. Oh- it tells me what page of how many pages there are. There is a NEXT (with an arrow) button, it looks like the fast-forward on my walkman. Click. I'm now at another page in this help section. It's telling me how to record my voice. The instructions are simple enough to understand, it's just the sequence of events that is long and appears complicated. I hope I can remember what I am supposed to do first when I'm trying this thing out. EXIT. Click. I'm going to try that RECORD VOICE button at the bottom. Click. A boxed area appears. There are new buttons - SPEAKER SOUND, REPLAY SOUND, RECORD SOUND. I click on SPEAKER SOUND first. Nothing happens. Click on REPLAY SOUND. Nothing. On RECORD SOUND it tells me to begin recording, but how do I hear it? I can't get into the HELP here!! In fact, I can't click on anything except the Record Box Buttons. How about EXIT? Click. O.K., it took away that boxed area. I think I'm back to the screen I was first on, with the letter boxes and the woman who appears to make a sound. Yes, it's the same screen. The instructions have appeared in that same place. 
So how do I get out of this activity? EXIT. Click. Good, I'm at the Main Menu. Now where was I again, before I got back to the Main Menu? Because I don't want to go into the same place. Was it Listening Activities? I wish there was something telling me where I had just been. At least with a book I could dog-ear it, or put in a book mark...I think I was at Speaking Activities. I don't know. I'll try CONVERSATIONS. Oh. More choices. How am I supposed to make a choice if I don't know what they are? There are six choices, and OBJECTIVES. I Click on OBJECTIVES. A small box appears and I read about speaking, listening, and reading skills in the Survival Skills-Academic area. A button at the top of this box that appeared (it wasn't there before) has a BACK TO MENU button. I like how the buttons change when my cursor passes over them. It makes me feel like the computer knows I'm here. O.K. I'll choose SURVIVAL SKILLS -ACADEMIC. What does survival mean? I look it up in my dictionary. It sounds like a jungle experience (survival), or war-zone thing. I wonder what the computer will do. Click. AAHHH! More choices! PICK A MODULE. What's a "module"? I'll just click on one of the boxes. And more choices! I don't know which one. SEE YOUR SELECTION NOW. SEE COMPLETE CONVERSATION. What's the difference? I'm picking the former. A video appears and I watch it. There's only a STOP button on the screen, and I can't see my cursor when I try to move it around the screen. I'll just watch the movie. There are a bunch of kids in a restaurant talking about classes and majors. There are a few white men, and an African American man, and an Asian looking woman. Now the film has stopped. A screen appears, filled with text. "Speaker" is at the left corner, and names of people are under there (I think they are names - Kisha, Joel, Melanie, Lee, Carl - I can't recognize any from the names I know as a Russian, except Carl, and we spell it with a K). There is text following each name. What do I do? There are a bunch of buttons across the bottom. There are ten. I recognize E- EXIT; M-MENU; 
H-HELP. But what are SCRIPT; C-CULTURE; V-WORDS; G-GRAMMAR; VPHRASES; VIDEO? I tried typing something, wow! Where am I now? Oh - it's the same screen as the speaking activities. How did I get here? I was just typing like this: owenlkdhjehldlld. Hmm. EXIT. Click. I'm back to that text screen I was on, with all the buttons at the bottom. I wish someone would suggest a route/what to do. CULTURE. Click. Same text, but there are highlighted strips of words. My cursor over those words makes the highlights change color. I accidentally click on one of those highlighted words. A small screen appears on the right. There is more text in it. How do I get rid of that small box? EXIT. Click. I'm back at the Main Menu! I don't get it! Now where was I again, before showing up here? I remember I had to make a lot of choices: Conversations, Survival Skills-Academic...And now I have to sit through the video again! I don't mind too much now, but later on, if I make a mistake and exit out, I'll have to sit through this thing over and over. I don't know how to bypass it. But wait, something is different this time. Now there is text under the video. STOP. Click. It doesn't change anything. I have no idea what to do now. Am I supposed to answer that question with one of those choices? I wasn't even paying attention. How do I run the video again? There is no HELP button, no directions. I'll click on one of those choices. O.K. The video is continuing. Now the screen appears, the one I had been trying to get back to, with all the text on it, and all the buttons at the bottom. 